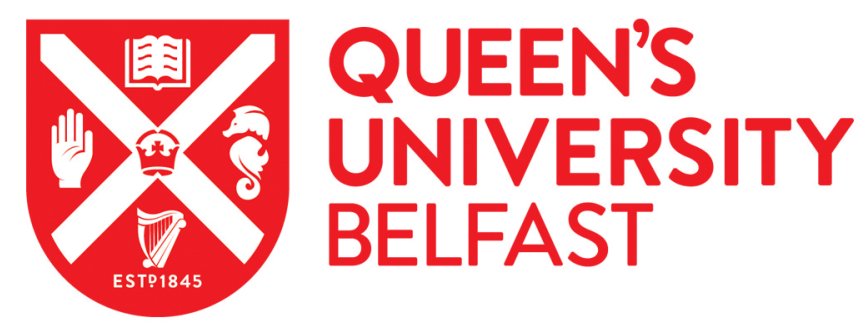

\title{
Finite Element Modelling of FRP Strengthened Restrained Concrete Slabs
}

\author{
Martin, A., Taylor, S., Robinson, D., \& Cleland, D. (2019). Finite Element Modelling of FRP Strengthened \\ Restrained Concrete Slabs. Engineering Structures, 187, 101-119. \\ https://doi.org/10.1016/j.engstruct.2019.02.035
}

\section{Published in:}

Engineering Structures

\section{Document Version:}

Peer reviewed version

\section{Queen's University Belfast - Research Portal:}

Link to publication record in Queen's University Belfast Research Portal

\section{Publisher rights}

Copyright 2019 Elsevier Ltd.

This manuscript is distributed under a Creative Commons Attribution-NonCommercial-NoDerivs License

(https://creativecommons.org/licenses/by-nc-nd/4.0/), which permits distribution and reproduction for non-commercial purposes, provided the author and source are cited.

\section{General rights}

Copyright for the publications made accessible via the Queen's University Belfast Research Portal is retained by the author(s) and / or other copyright owners and it is a condition of accessing these publications that users recognise and abide by the legal requirements associated with these rights.

Take down policy

The Research Portal is Queen's institutional repository that provides access to Queen's research output. Every effort has been made to ensure that content in the Research Portal does not infringe any person's rights, or applicable UK laws. If you discover content in the Research Portal that you believe breaches copyright or violates any law, please contact openaccess@qub.ac.uk. 


\title{
Finite Element Modelling of FRP Strengthened Restrained Concrete Slabs
}

\begin{abstract}
This paper considers the use of Nonlinear Finite Element Analysis (NLFEA) to predict the load capacity of a range of experimentally tested in-plane restrained reinforced concrete slabs which experienced internal arching effects under loading. The slabs were constructed at one third scale and strengthened with basalt fibre reinforced polymer (BFRP) or carbon fibre reinforced polymer (CFRP) bonded in place using the near surface mounted (NSM) technique. As the research was representative of existing floor slabs within reinforced concrete building frames, all test specimens were constructed with normal strength concrete $\left(\sim 40 \mathrm{~N} / \mathrm{mm}^{2}\right)$ and $0.15 \%$ steel reinforcement. One tenth of one percent fibre reinforced polymer (FRP) was used to strengthen samples which were compared with unstrengthened control specimens. The London University Structural Analysis System (LUSAS) finite element analysis software package was used to model all test samples using experimentally derived material test values.

Experiments and NLFEA models were compared with the Queen's University Belfast (QUB) arching theory which showed that LUSAS was slightly more accurate than the QUB arching theory in predicting slab capacity. However, the QUB arching theory was found to be slightly more consistent in estimating slab capacities compared with LUSAS. Yet, both of these methods were significantly better at predicting slab capacities than existing Eurocode and American Concrete Institute codes.
\end{abstract}

\section{Keywords}

Nonlinear Finite Element Analysis, NLFEA, LUSAS, Near surface mounted, NSM, Fibre reinforced polymer, basalt fibre reinforced polymer, carbon fibre reinforced polymer, FRP, BFRP, CFRP, strengthening, concrete, in-plane lateral restraint, arching, compressive membrane action.

\section{Introduction}

With estimates that the built environment is directly attributable to $40 \%$ of greenhouse gasses [1] and that $87 \%$ of buildings in existence in 2050 have already been constructed [2], there has been significant recent interest [3][4] in repurposing 
buildings through 'adaptive reuse'. However, due to the nature of their bespoke design, it may be necessary to carry out strengthening work on structural elements within a building in order to increase their load carrying capacity. One possible method of achieving this is to bond fibre reinforced polymers (FRPs) to the existing concrete floor slabs. Due to their low weight and high resistance to corrosion from de-icing salts, FRPs have been used in strengthening road bridges [5], although they are now considered as a viable option for use in strengthening building structures using methods such as conventional adhesive attachment (CAA) [6-8], near surface mounting (NSM) [9][10] or by plate fastening [11]. Of these approaches, NSM is viewed as being particularly advantageous due to causing minimal intrusion.

Capacity increases may also be achieved by considering the effects of restraint and internal arching effects within concrete slabs. These are typically not considered by design engineers prior to construction. However, by accounting for these effects retrospectively, it may be possible to 'find' additional capacity to permit adaptive reuse to proceed. Various methods have been developed since the early $20^{\text {th }}$ century [1226] to quantify these effects. However, they have not been adopted within modern European [27][28] and American [29] building design codes, although they do feature within some specialist highway bridge design codes [30-32].

A review of the literature has shown that whilst there has been a significant amount of research surrounding the use of FRPs, their strengthening effects have not been considered in the presence of in-plane restraint which causes the development of arching or compressive membrane action within concrete slabs. Consequentially, investigations into the suitability of non-linear finite element analysis (NLFEA) software to predict their behaviour in these circumstances has also not been carried out.

\subsection{Background}

\subsubsection{Fibre reinforced polymer strengthening}

NSM strengthening of existing reinforced concrete structures can be traced back to strengthening bridge slabs with grouted steel reinforcement [33]. Whilst strengthening using steel bars continues to be of interest [34], the use of FRPs has gained more interest in recent times [35]. Some bridges have also been built entirely or partially from FRPs [36]. FRPs also offer faster construction, higher strengths, lower weights, and greater durability compared with steel. However, their main perceived drawbacks are their high initial costs and lower elastic moduli. 
Within this research, basalt and carbon fibre reinforced polymers were used as strengthening materials. Basalt fibres are obtained by melting basalt; one of the most common rocks in the earth's crust; at $1300-1700{ }^{\circ} \mathrm{C}$ and spinning the molten liquid [37] into thin fibres. However, the mechanical properties of these fibres are dependent, to an extent, on the origin of the raw material and the exact processes employed in their production. Carbon fibres, on the other hand, were first produced in 1958 [38] during carbon arc experimentation under high temperatures and pressures [39]. Since their original discovery, industrial methods to produce them have also been refined. FRP bars containing carbon or basalt fibres are then typically manufactured with either circular or rectangular cross sections using a pultrusion process to suspend the fibres within a polymer resin and a sand coating may be added to aid with cohesion in concrete.

\subsubsection{Compressive membrane action}

If the edges of a concrete slab are restrained against lateral movement, internal arching develops as the slab deflects [20], as shown in Figure 1. This arching behaviour is known as compressive membrane action (CMA) and has been shown to enhance the flexural and shear capacity of reinforced concrete slabs.

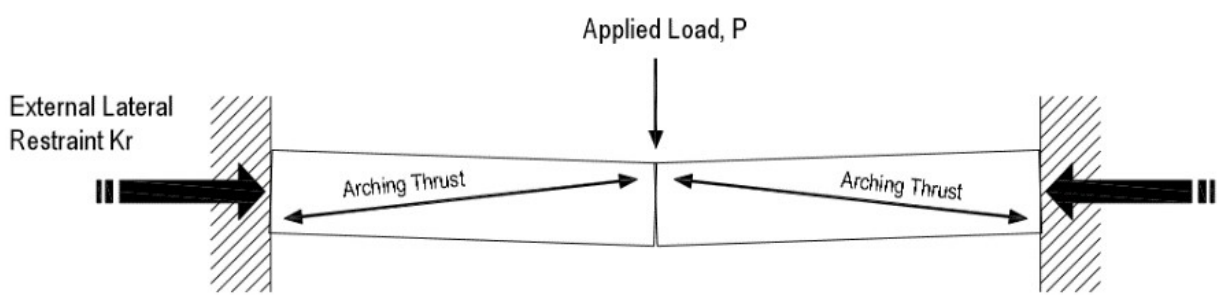

Figure 1: Arching Action in Laterally Restrained Slabs

In the early part of the 20th century, the strength enhancing effects of arching action, above those predicted by flexural analysis, were first recognised [12]. However, it was not until the 1950s when Ockleston [13][14] carried out full scale destructive tests on a three storey reinforced concrete building in Johannesburg that serious attempts to quantify arching were carried out. Since then, theories have been developed to explain arching, primarily by McDowell et al. [15] and Park [16-18]. More recently, researchers at Queen's University Belfast [19-26] have built upon work by McDowell et al. [15] for application to restrained concrete slabs experiencing internal arching effects. 


\subsubsection{Queen's University Belfast arching theory}

The Queen's University Belfast (QUB) arching theory was initially developed by Rankin [19] and later published by Rankin and Long [20]. Taylor [21] subsequently refined the concrete compressive stress block parameters for application of the theory to high strength concrete. In the Rankin and Long [20] approach, the degree of lateral restraint was dealt with using a three-hinged arch analogy, whereby the behaviour of an arch with elastic spring restraints was equated to the behaviour of a longer rigidly restrained arch, as shown in Figure 2.

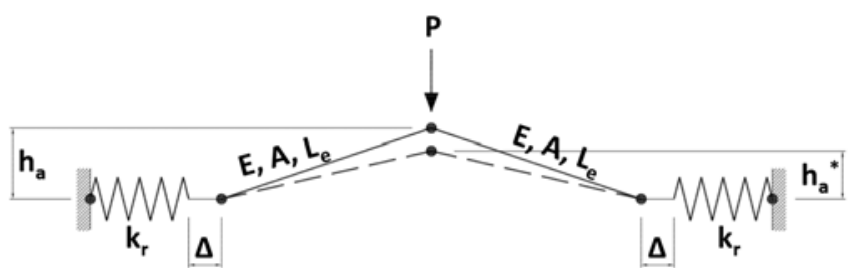

Figure 2(a): Elastically restrained three pinned arch [20]

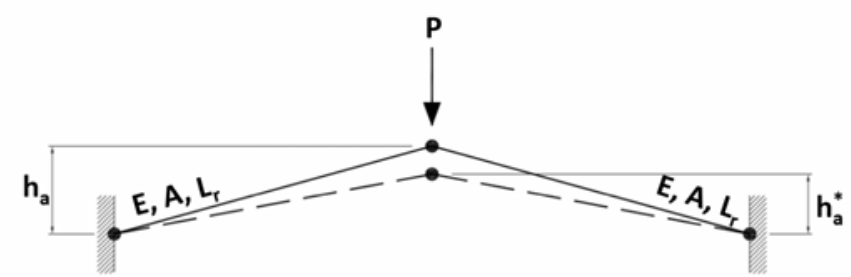

Figure 2(b): Equivalent rigidly restrained three pinned arch [20]

The prediction of ultimate capacity was based on the deformation theory of McDowell et al. [15]. The effects of arching and bending were considered separately, although in reality compression in concrete was due to the action of both arching and bending. This arching analysis was further developed by Taylor et al. [22][23] for bridge deck slabs with high strength concrete $\left(>70 \mathrm{~N} / \mathrm{mm}^{2}\right)$. To take account of the less than rigid restraint, an 'equivalent' rigidly restrained arch length, $L_{r}$, is used:

$$
L_{r}=L_{e} \sqrt[3]{\left(\frac{E_{c} A}{K L_{e}}\right)+1}
$$

where: $\quad L_{r}=$ Half span of equivalent rigidly restrained slab strip

$L_{e}=$ Half span of 'real' strip of slab with finite lateral restraint

$A=$ Area of concrete due to arching

$k_{r}=$ Stiffness of elastic spring restraint 
Tests have previously shown good correlation between the QUB arching theory and experimental values [25][26]. Also, in recent years, several international bridge design codes [30-32] have incorporated design guidance to include the beneficial effects of CMA in bridge deck slab design. The procedure for assessing the strength of laterally restrained slabs using the QUB arching theory is outlined below:

\section{Calculating the strength of an in-plane restrained slab}

The process of establishing the depth available for arching is iterative. Hence, the flow chart in Figure 3 illustrates the process involved in evaluating the strength of a laterally restrained slab.

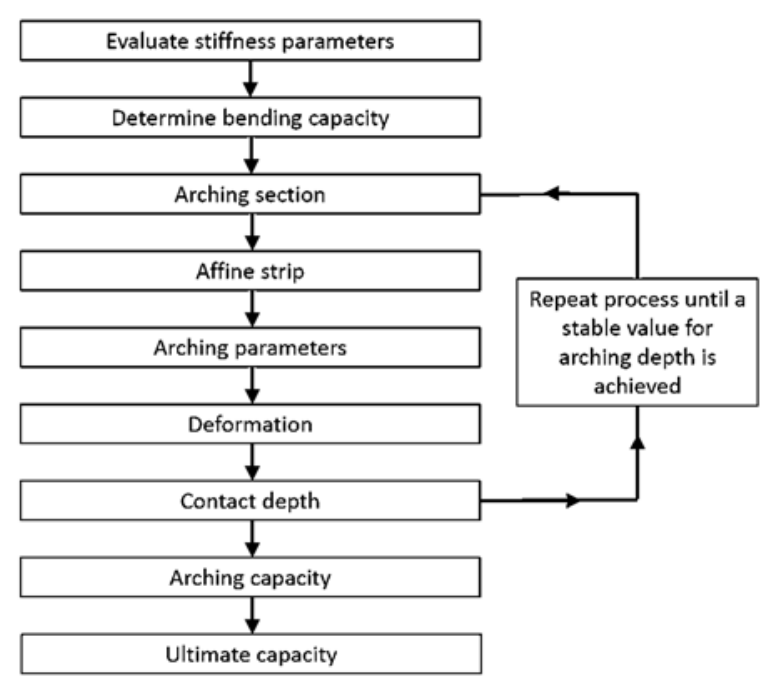

Figure 3: Flow chart illustrating iterative procedure to determine in-plane restrained slab capacity

\section{Step 1}

\section{Stiffness parameters}

Evaluation of the restraint stiffness, $k_{r}$, in experimental slab specimens due to the presence of in-plane restraint beams was based upon an analysis of electrical resistance strain (ERS) gauge readings within restraint beam reinforcement bars and slab movements.

\section{Bending capacity}

Bending capacity of the rectangular cross section is based upon the original approach developed by Rankin [19] and Taylor [21], with the additional inclusion of FRP bars along with steel reinforcement, as shown in Figure 4. 
Proportional depth of stress block factor: $\quad \beta=\mathbf{1}-\mathbf{0 . 0 0 3} f_{\text {ck,cube }} \quad$ but $\leq \mathbf{0 . 9}$

Depth of neutral axis:

$$
x=\frac{A_{S} f_{y k}+A_{F R P} f_{F R P, k}}{0.67 f_{c k, c u b e} \beta b}
$$

Moment capacity due to bending:

$$
M_{b}=A_{S} f_{y k}\left[d-\frac{\beta x}{2}\right]+A_{F R P} f_{F R P, k}\left[d_{F R P}-\frac{\beta x}{2}\right]
$$

In all cases, the partial safety factors for steel and FRP materials are unity.

As loads are considered as midspan knife edge loads, the bending moment $M_{b}$ can be related to an equivalent knife edge load, $\mathrm{P}_{\mathrm{b}}$, using equation (5).

$$
P_{b}=\frac{4 M_{b}}{L}
$$
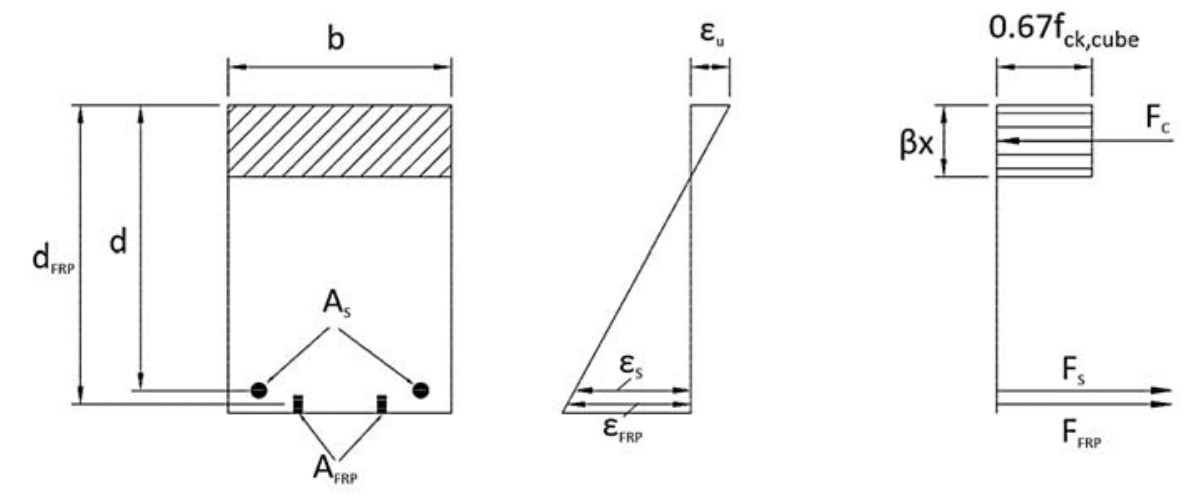

Figure 4: Rectangular section stress distribution for bending component within QUB Arching

\section{Step 2}

\section{Arching section}

Depth available for arching, established by iteration: $\quad d_{1}=\frac{h-2 \beta x}{2}$

\section{Affine strip}

Area of concrete due to arching: $\quad \boldsymbol{A}=\boldsymbol{\alpha} \boldsymbol{b} \boldsymbol{d}_{1}$

$$
\alpha=1-\frac{u}{2}
$$

where: $\alpha=1$ for the first iteration, which is reflective of zero deflection $u=$ McDowell's [15] non-dimensional arching deflection parameter 
Equivalent rigid half arch span: $\quad L_{r}=L_{e}\left[\frac{E_{C} A}{k_{r} L_{e}}+1\right]^{1 / 3}$

where: $L_{e}=$ Half of the actual slab span

$k_{r}=$ Axial restraint stiffness

\section{Arching parameters}

Ultimate compressive strain in concrete:

$$
\varepsilon_{u}=0.0043-\left[f_{c k, c u b e}-60\right] \times\left(2.5 \times 10^{-5}\right) \quad \text { but } \leq 0.0043
$$

Concrete plastic strain: $\quad \varepsilon_{c}=2 \varepsilon_{u}(1-\beta)$

McDowell's [15] non-dimensional geometry and material factor:

$$
R=\frac{\varepsilon_{c} L_{r}^{2}}{4 d_{1}^{2}}
$$

\section{Deformation}

$$
\begin{array}{lll}
\text { For } & 0<R \leq 0.26 & u=-0.15+0.36 \sqrt{0.18+5.6 R} \\
\text { For } & R>0.26 & u=0.31
\end{array}
$$

\section{Contact depth}

With a value of $u$ established, it is then possible to determine a refined value for the contact depth from equation (8), area of concrete due to arching from equation (7), equivalent rigid half arch span from equation (9), McDowell's non-dimensional geometry and material factor from equation (12) and back to a newly refined value for the contact depth from equation (8) before the iterative process repeats until equilibrium is established.

\section{Step 3}

\section{Arching capacity}

For $\quad 0<R \leq 0.26, \quad$ Moment ratio $\quad M_{r}=4.3-16.1 \sqrt{\left(3.3 \times 10^{-4}\right)+0.1243 R}$

For $\quad R>0.26, \quad$ Moment ratio $\quad M_{r}=\frac{0.3615}{R}$ 
The equivalent rigid arching moment of resistance is expressed as:

$$
M_{a r}=0.168 b f_{c k, c u b e} d_{1}^{2} M_{r}\left(\frac{L_{e}}{L_{r}}\right)
$$

However, the elastic arching moment of resistance is expressed as:

$$
M_{a}=M_{a r}\left(\frac{L_{e}}{L_{r}}\right)
$$

As loads are considered as midspan knife edge loads, the bending moment due to arching, $M_{a}$, can be related to an equivalent knife edge load, $P_{a}$, using equation (19).

$$
P_{a}=\frac{4 M_{a}}{L}
$$

\section{Ultimate capacity}

$$
\boldsymbol{P}_{p}=\boldsymbol{P}_{a}+\boldsymbol{P}_{b}
$$

In recent years, the UK highways agency design manual for roads and bridges [30], the American Association of State Highway and Transportation Officials Load and Resistance Factor Design Bridge Design Specifications [31] and the Canadian Highway Bridge Design Code [32] have incorporated design guidance to include the beneficial effects of CMA in bridge deck slab design. The procedure for assessing the strength of laterally restrained slabs has been outlined in detail by Tharmarajah [26].

\subsubsection{Nonlinear finite element modelling}

As the restraining effects of a reinforced concrete frame on insitu floor slabs is a common occurrence within many building structures, this investigation sought to establish whether FRP strengthening and in-plane restraint had any cumulative effect in increasing slab capacity. To do this, experimental test specimens were designed, constructed and tested to failure. However, an additional strand of the research was to compare test results, QUB arching theory predictions, Eurocode and American Concrete Institute code predictions with those obtained using computer model predictions. Hence, nonlinear finite element analysis (NLFEA) models were developed.

NLFEA originated in the 1940s with Hrennikoff [40] and Courant [41] as a means to solve complex engineering analysis problems involving elasticity. Over a decade later Turner [42] developed the finite element method as an extension of matrix structural analysis while investigating the stiffness and deflection of aircraft wings. In addition, 
other researchers such as Argyris [43], Zienkiewicz [44-48] and Martin [49], in the field of computational analysis of engineering structures, are also credited with developing aspects of modern finite element analysis at around the same time as Turner. Today, following continual advances in software and hardware development, there are a wide range of NLFEA packages available to suit a variety of engineering disciplines and applications. For this research, the London University Structural Analysis System (LUSAS) NLFEA package was adopted, as initial investigations showed that this software was particularly effective in simulating slab stiffness reductions due to concrete cracking; thus producing initial load-deflection output reflective of experimental behaviour.

The underlying benefit of carrying out NLFEA investigations into in-plane restrained slabs was to establish parameters which could be used in constructing computer models of FRP strengthened and unstrengthened in-plane restrained slabs beyond the scope of those physically tested within the current research; with a reasonable degree of confidence in how they would behave. By benchmarking the behaviour of physical test samples against computer simulations, these investigations aimed to illustrate the practical benefits to practising design engineers of adopting the software and guidelines established for modelling 'real' slabs within existing reinforced concrete building frames, leading to greater sustainability within the construction industry.

\subsection{Objectives of the research}

The objective of the research is to compare the behaviour and predicted capacity of a range of reinforced concrete test slabs with varying levels of in-plane restraint and FRP strengthening with corresponding NLFEA models. Guidelines on constructing NLFEA models for FRP strengthened in-plane restrained slabs can then be established for application to slabs of this type within existing building structures. Comparisons can also be made between computer simulations, European and American design code predictions and the QUB arching theory hand calculation technique. 


\section{Experimental investigations}

\subsection{Material Properties}

\subsubsection{Concrete}

Normal strength concrete with a target strength of $40 \mathrm{~N} / \mathrm{mm}^{2}$ was used throughout the research, with a mix outlined in Table 1 . The use of $6 \mathrm{~mm}$ aggregate avoided problems associated with size effects on shear behaviour and cracking in one-third scale test slabs.

Table 1: Concrete Mix Design

\begin{tabular}{|c|c|c|c|c|c|c|}
\hline $\begin{array}{l}\text { Water } \\
\left(\mathrm{kg} / \mathrm{m}^{3}\right)\end{array}$ & $\begin{array}{l}\text { Cement } \\
\left(\mathrm{kg} / \mathrm{m}^{3}\right)\end{array}$ & $\begin{array}{c}6 \mathrm{~mm} \\
\text { Aggregate } \\
\left(\mathrm{kg} / \mathrm{m}^{3}\right)\end{array}$ & $\begin{array}{c}\text { Coarse } \\
\text { Grit } \\
\left(\mathrm{kg} / \mathrm{m}^{3}\right)\end{array}$ & $\begin{array}{c}\text { Zone } 2 \\
\text { Sand } \\
\left(\mathrm{kg} / \mathrm{m}^{3}\right)\end{array}$ & $w / b$ & $\begin{array}{c}\text { Target 28-Day Cube } \\
\text { Compressive } \\
\text { Strength (MPa) }\end{array}$ \\
\hline 250 & 400 & 525 & 875 & 350 & 0.625 & 40.00 \\
\hline
\end{tabular}

Compressive cube tests and tensile splitting tests were carried out on hardened concrete [50-52] using a calibrated testing machine [53]. The elastic modulus of concrete, $E_{c}$, was established using Hognestad's [54] relationship, outlined in equation (21).

$$
\left.\boldsymbol{E}_{\boldsymbol{c}}=4230 \sqrt{\boldsymbol{f}_{\text {ck,cube }}} \quad \text { (for } \mathrm{E}_{\mathrm{c}} \text { in } \mathrm{N} / \mathrm{mm}^{2}\right)
$$

where: $E_{c}=$ Elastic modulus of concrete $\left(\mathrm{N} / \mathrm{mm}^{2}\right)$

$$
f_{c k, c u b e}=\text { Compressive cube strength of concrete }\left(\mathrm{N} / \mathrm{mm}^{2}\right)
$$

\subsubsection{Steel reinforcement}

A limited supply of $6 \mathrm{~mm}$ diameter straight bars were used along with $6 \mathrm{~mm}$ diameter bars from mesh, which were easier to source, in most test specimens. Thirty-two millimetre diameter straight bars were also used in one test specimen in the research. All bars were 'ribbed' [55] and were tested under tension at $0.2 \mathrm{kN} / \mathrm{s}$ [56]. Reinforcement properties are summarised in Table 2. 
Table 2: Reinforcement properties

\begin{tabular}{|c|c|c|c|c|c|}
\hline Bar Diameter & $\begin{array}{c}\text { Upper } \\
\text { Yield, } R_{\mathrm{eH}} \\
\left(\mathrm{N} / \mathrm{mm}^{2}\right) \\
\end{array}$ & $\begin{array}{l}\text { Lower Yield, } R_{\mathrm{el}} \\
\quad\left(\mathrm{N} / \mathrm{mm}^{2}\right)\end{array}$ & $\begin{array}{c}0.2 \% \text { proof } \\
\text { strength, } \mathrm{R}_{\mathrm{p} 0.2} \\
\left(\mathrm{~N} / \mathrm{mm}^{2}\right)\end{array}$ & $\begin{array}{c}\text { Yield } \\
\text { Strength, } \mathrm{f}_{\mathrm{yk}} \\
\left(\mathrm{N} / \mathrm{mm}^{2}\right)\end{array}$ & $\begin{array}{c}\text { Elastic } \\
\text { modulus, } \mathrm{E}_{\mathrm{s}} \\
\left(\mathrm{N} / \mathrm{mm}^{2}\right) \\
\end{array}$ \\
\hline 6mm (Straight) & 499 & 483 & - & 499 & $205.5 \times 10^{3}$ \\
\hline $6 \mathrm{~mm}$ (Mesh) & - & - & 549 & 549 & $201.9 \times 10^{3}$ \\
\hline 32mm (Straight) & - & - & - & 485 & $200 \times 10^{3}$ \\
\hline
\end{tabular}

\subsubsection{FRP bars}

For each FRP bar type, representative samples were tested under tension at $0.2 \mathrm{kN} / \mathrm{s}$, with attached electronic resistance strain (ERS) gauges [56]. FRP properties are summarised in Table 3.

Table 3: FRP properties

\begin{tabular}{|c|c|c|c|}
\hline FRP Type & $\begin{array}{c}\text { Cross Sectional Area } \\
\left(\mathbf{m m}^{\mathbf{2}} \mathbf{)}\right.\end{array}$ & $\begin{array}{c}\text { Rupture Strength } \\
\left(\mathbf{N} / \mathbf{m m}^{\mathbf{2}}\right)\end{array}$ & Elastic Modulus (N/mm $\mathbf{~})$ \\
\hline CFRP & 19.65 & 990 & 77452 \\
\hline BFRP & 13.34 & 1138 & 35025 \\
\hline
\end{tabular}

\subsection{Estimation of in-plane restraint stiffness of existing slab}

As the degree of in-plane restraint affects the level of compressive membrane action, stiffness ratios of zero (i.e. simply supported), 'regular', ' $0.5 x$ regular', ' $2 x$ regular' and ' $4 \mathrm{x}$ regular' were used; where 'regular' restraint was considered as that experienced by a typical beam and slab shown in Figure 5 with details outlined in Table 4.

Table 4: 'Regular' restrained slab details

\begin{tabular}{|c|c|}
\hline Elastic modulus of steel, $\mathbf{E}_{\mathbf{s}}$ & $210000 \mathrm{~N} / \mathrm{mm}^{2}$ \\
\hline Elastic modulus of concrete, $\mathbf{E}_{\mathbf{c}}$ & $26753 \mathrm{~N} / \mathrm{mm}^{2}$ \\
\hline Tie Beam Width & $300 \mathrm{~mm}$ \\
\hline Tie Beam Depth & $500 \mathrm{~mm}$ \\
\hline Total Tie Beam Reinforcement & 6 No. 25mm Diameter Bars (2\% by area) \\
\hline Slab Width & $5000 \mathrm{~mm}$ \\
\hline Slab Depth & $250 \mathrm{~mm}$ \\
\hline Total Slab Reinforcement & $0.15 \%$ Slab Cross Sectional Area \\
\hline
\end{tabular}



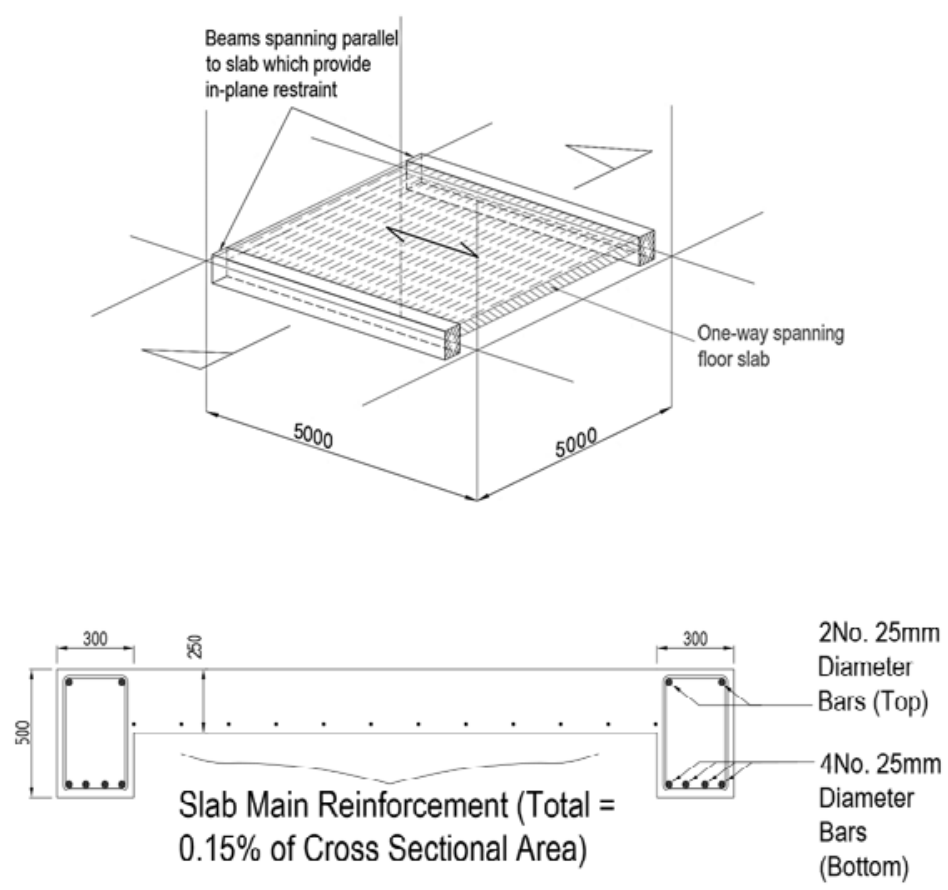

Figure 5: 'Regular' in-plane restraint arrangement in a typical building frame

In this case, $E_{c}$ was assumed to be directly related to compressive cube strength, $\mathrm{f}_{\mathrm{ck}, \text { cube }}\left(40 \mathrm{~N} / \mathrm{mm}^{2}\right)$, using the Hognestad [54] relationship outlined in equation (21). Based on the 'regular' restrained slab shown in Figure 5; and using $E_{s}$ and $E_{c}$ values in Table 4; slab stiffness $k_{s}$, restraint stiffness $k_{r}$ and stiffness ratio $r$ were evaluated for a 'regular' level of in-plane restraint stiffness, assuming uncracked (i.e. full cross sectional utilisation of concrete) behaviour in the following manner:

$$
\begin{aligned}
& k_{S}=k_{\text {slab }}=\frac{E_{c, \text { Slab }} A_{c, \text { Slab }}}{L_{\text {Slab }}}+\frac{E_{S, \text { Slab }} A_{S, \text { Slab }}}{L_{\text {Slab }}}+\frac{E_{F R P} A_{F R P}}{L_{\text {Slab }}}=6756968 \mathrm{~N} / \mathrm{mm} \\
& k_{r}=k_{\text {Beam }}=\frac{E_{c, \text { Beam }} A_{c, \text { Beam }}}{L_{\text {Beam }}}+\frac{E_{S, \text { Beam }} A_{S, \text { Beam }}}{L_{\text {Beam }}}=1821063 \mathrm{~N} / \mathrm{mm} \\
& \boldsymbol{r}=\frac{k_{r}}{k_{S}}=\frac{1821063}{6756968}=\mathbf{0 . 2 7 0}
\end{aligned}
$$

The following 'uncracked' stiffness ratios were chosen for the investigation of slabs with a span-to-depth ratio of 20 :

- 'Regular' restraint, $r_{\text {uncracked }}=0.270$

- $1 / 2$ Regular' restraint, $r_{\text {uncracked }}=0.135$

- ' $2 \times$ Regular' restraint, $r_{\text {uncracked }}=0.540$ 
For slabs with a span-to-depth ratio of 15 , the following stiffness ratios were considered:

- Regular' restraint, $r_{\text {uncracked }}=0.202$

- ' $2 \times$ Regular' restraint, $r_{\text {uncracked }}=0.404$

- '4× Regular' restraint, $r_{\text {uncracked }}=0.808$

\subsection{Test slabs}

Seventeen experimental test slabs were cast with varying levels of in-plane restraint which was provided by casting side restraint beams with varying sizes and reinforcement quantities to achieve the range of restraint levels required. These beams were separated by a $50 \mathrm{~mm}$ gap from slab regions, as shown in Figures 6 and 7. Unrestrained samples were simply supported rectangular units. Test slabs were coded to indicate restraint level, FRP and span-to-depth ratio as outlined below:

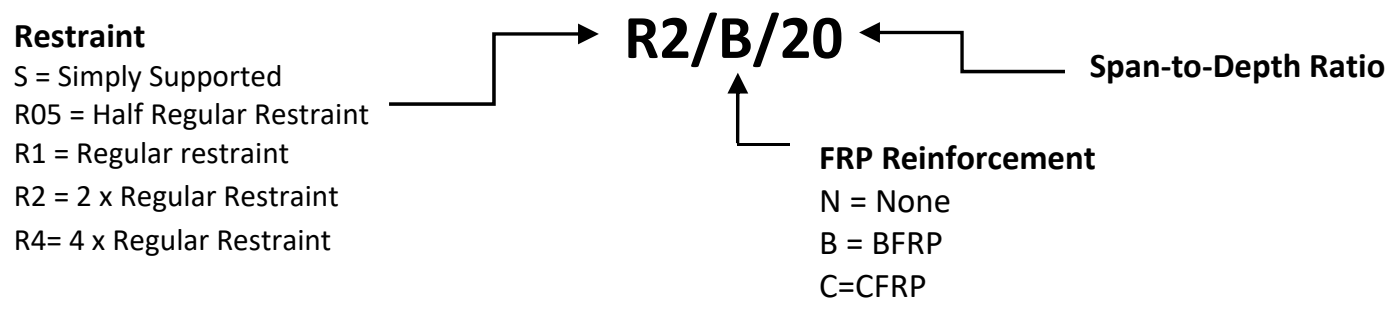

Slab steel reinforcement was set slightly above minimum design code required levels at $0.15 \%$, as this was considered representative of domestically loaded floor slabs. All dimensions and reinforcement data are outlined in Figure 7 and Table 5 respectively.

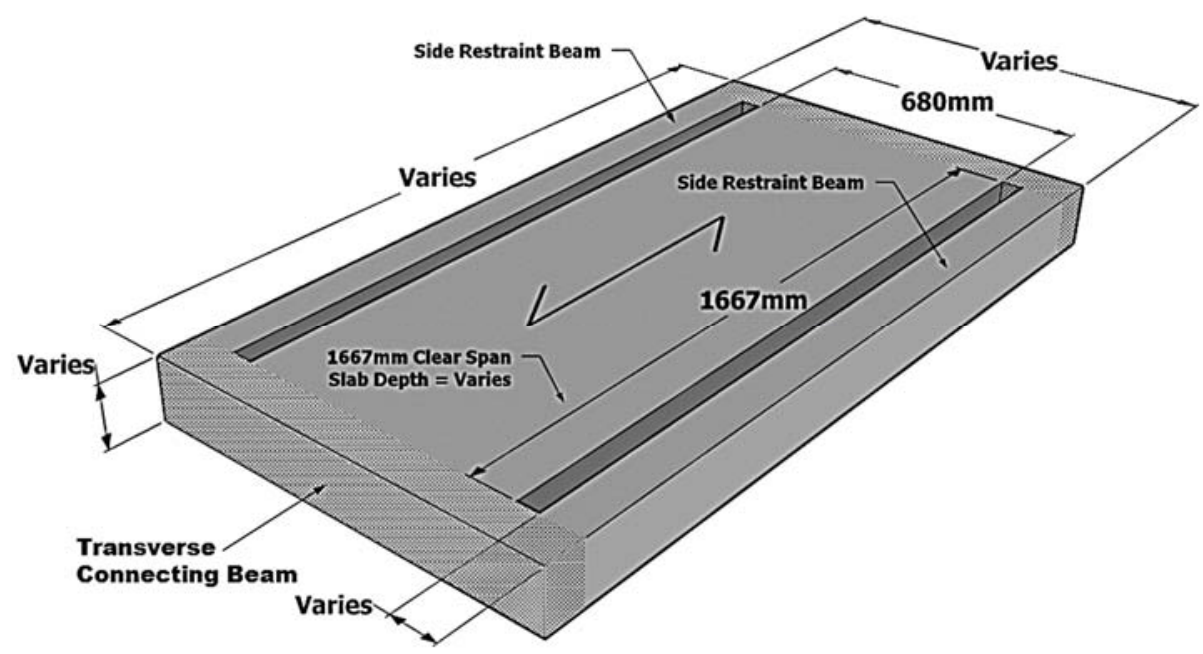

Figure 6: Typical Test Slab Geometry 


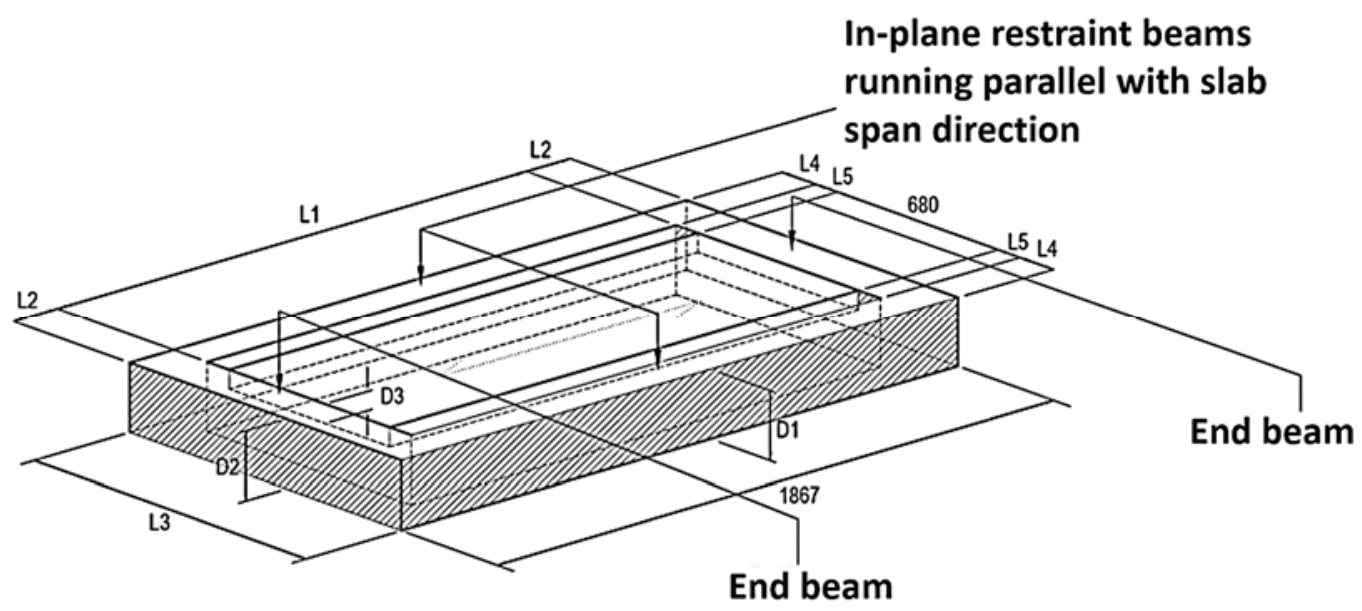

Figure 7: Typical In-plane restrained test slab (dimensions shown detailed in Table 5) 
Table 5: Slab dimensions and reinforcement

\begin{tabular}{|c|c|c|c|c|c|c|c|c|c|c|c|c|c|c|c|c|c|}
\hline \multirow[t]{2}{*}{ Slab Code } & $\begin{array}{c}\text { Clear } \\
\text { Span } \\
\text { of }\end{array}$ & $\begin{array}{l}\text { Width } \\
\text { of End } \\
\text { Beams, }\end{array}$ & $\begin{array}{c}\text { Total } \\
\text { Width } \\
\text { of }\end{array}$ & $\begin{array}{l}\text { Width of } \\
\text { In-plane } \\
\text { Restraint }\end{array}$ & $\begin{array}{l}\text { Width of } \\
\text { Gap } \\
\text { Between } \\
\text { Slab and } \\
\text { In-plane }\end{array}$ & $\begin{array}{c}\text { Total } \\
\text { Depth of } \\
\text { In-plane } \\
\text { Restraint }\end{array}$ & $\begin{array}{l}\text { Total } \\
\text { Depth } \\
\text { of End }\end{array}$ & $\begin{array}{l}\text { Total } \\
\text { Depth } \\
\text { of }\end{array}$ & \multicolumn{2}{|c|}{ Slab Steel Reinforcement } & \multirow{2}{*}{$\begin{array}{l}\text { Percentage } \\
\text { Steel } \\
\text { Reinforcement } \\
\text { in Slab }\end{array}$} & \multirow{2}{*}{$\begin{array}{c}\text { Effective Depth } \\
\text { of } \\
\text { Reinforcement } \\
\text { in Slab } \\
\\
(\mathrm{mm})\end{array}$} & \multicolumn{2}{|c|}{$\begin{array}{l}\text { Steel Reinforcement in } \\
\text { Restraint Beams }\end{array}$} & \multicolumn{2}{|c|}{$\begin{array}{l}\text { Steel Reinforcement in } \\
\text { End Beams }\end{array}$} & \multirow[t]{2}{*}{$\begin{array}{l}\text { Strengthening } \\
\text { Material }\end{array}$} \\
\hline & $(\mathrm{mm})$ & $(\mathrm{mm})$ & $(\mathrm{mm})$ & $(\mathrm{mm})$ & $(\mathrm{mm})$ & $(\mathrm{mm})$ & $(\mathrm{mm})$ & $(\mathrm{mm})$ & & Bar Type & & & & Bar Type & & Bar Type & \\
\hline$S / N / 20$ & 1867 & 0 & 680 & 0 & 0 & 0 & 0 & 83.3 & 3 No. $6 \mathrm{~mm} \phi$ & Straights & 0.15 & 65.3 & None & NA & None & NA & N/A \\
\hline$S / N / 15$ & 1867 & 0 & 680 & 0 & 0 & 0 & 0 & 111.1 & 4 No. $6 \mathrm{~mm} \phi$ & Straights & 0.15 & 93.1 & None & NA & None & NA & N/A \\
\hline$s / c / 20$ & 1867 & 0 & 680 & 0 & 0 & 0 & 0 & 83.3 & 3 No. $6 \mathrm{~mm} \phi$ & Mesh & 0.15 & 65.3 & None & NA & None & NA & $\begin{array}{c}\text { 3No.CFRP @ } \\
19.65 \mathrm{~mm}^{2}\end{array}$ \\
\hline$S / B / 20$ & 1867 & 0 & 680 & 0 & 0 & 0 & 0 & 83.3 & 3 No. $6 \mathrm{~mm} \phi$ & Straights & 0.15 & 65.3 & None & NA & None & NA & $\begin{array}{c}\text { 6No. BFRP @ } \\
12.34 \mathrm{~mm}^{2} \\
\end{array}$ \\
\hline $\mathrm{S} / \mathrm{B} / 15$ & 1867 & 0 & 680 & 0 & 0 & 0 & 0 & 111.1 & 4 No. $6 \mathrm{~mm} \phi$ & Straights & 0.15 & 93.1 & None & NA & None & NA & $\begin{array}{c}\text { 8No. BFRP @ } \\
12.34 \mathrm{~mm}^{2}\end{array}$ \\
\hline $\mathrm{R} 1 / \mathrm{N} / \mathbf{2 0}$ & 1667 & 100 & 920 & 70 & 50 & 100 & 100 & 83.3 & 3 No. $6 \mathrm{~mm} \phi$ & Straights & 0.15 & 65.3 & 4 No. $6 \mathrm{~mm} \mathrm{\phi}$ & Straights & 4 No. $6 \mathrm{~mm} \mathrm{\phi}$ & Straights & N/A \\
\hline $\mathrm{R} 0.5 / \mathrm{N} / 20$ & 1667 & 100 & 880 & 50 & 50 & 60 & 83.3 & 83.3 & 3 No. $6 \mathrm{~mm} \phi$ & Straights & 0.15 & 65.3 & 4 No. $6 \mathrm{~mm} \phi$ & Straights & 4 No. $6 \mathrm{~mm} \mathrm{\phi}$ & Straights & N/A \\
\hline $\mathrm{R} 2 / \mathrm{N} / 20$ & 1667 & 100 & 980 & 100 & 50 & 147 & 147 & 83.3 & 3 No. $6 \mathrm{~mm} \phi$ & Straights & 0.15 & 65.3 & $4 \mathrm{No} .6 \mathrm{~mm} \phi$ & Straights & $4 \mathrm{No} .6 \mathrm{~mm} \mathrm{\phi}$ & Straights & N/A \\
\hline $\mathrm{R} 2 / \mathrm{N} / 15$ & 1667 & 100 & 980 & 100 & 50 & 143 & 143 & 111.1 & 4No. $6 \mathrm{~mm} \phi$ & Straights & 0.15 & 93.1 & 4 No. $6 \mathrm{~mm} \phi$ & Straights & 4 No. $6 \mathrm{~mm} \phi$ & Straights & N/A \\
\hline $\mathrm{R} 4 / \mathrm{N} / 15$ & 1667 & 100 & 980 & 100 & 50 & 143 & 143 & 111.1 & 4 No. $6 \mathrm{~mm} \phi$ & Mesh & 0.15 & 93.1 & 2 No. $32 \mathrm{~mm} \phi$ & Bent Bars & $\begin{array}{c}2 \text { No. } 32 \mathrm{~mm} \\
\phi\end{array}$ & Bent Bars & N/A \\
\hline $\mathrm{R} 1 / \mathrm{C} / 20$ & 1667 & 100 & 920 & 70 & 50 & 100 & 100 & 83.3 & 3 No. $6 \mathrm{~mm} \phi$ & Mesh & 0.15 & 65.3 & 4 NNo. $6 \mathrm{~mm} \phi$ & Mesh & 4 No. $6 \mathrm{~mm} \phi$ & Mesh & $\begin{array}{l}\text { 3No.CFRP @ } \\
19.65 \mathrm{~mm}^{2}\end{array}$ \\
\hline $\mathrm{R} 1 / \mathrm{B} / \mathbf{2 0}$ & 1667 & 100 & 920 & 70 & 50 & 100 & 100 & 83.3 & 3 No. $6 \mathrm{~mm} \phi$ & Mesh & 0.15 & 65.3 & 4No. $6 \mathrm{~mm} \mathrm{\phi}$ & Mesh & 4No. $6 \mathrm{~mm} \mathrm{\phi}$ & Mesh & $\begin{array}{c}\text { 6No. BFRP @ } \\
12.34 \mathrm{~mm}^{2}\end{array}$ \\
\hline $\mathrm{R} 0.5 / \mathrm{C} / 20$ & 1667 & 100 & 880 & 50 & 50 & 60 & 83.3 & 83.3 & 3 No. $6 \mathrm{~mm} \phi$ & Mesh & 0.15 & 65.3 & 4 No. $6 \mathrm{~mm} \phi$ & Mesh & 4 No. $6 \mathrm{~mm} \phi$ & Mesh & $\begin{array}{l}\text { 3No.CFRP@ } \\
19.65 \mathrm{~mm}^{2}\end{array}$ \\
\hline $\mathrm{R} 0.5 / \mathrm{B} / 20$ & 1667 & 100 & 880 & 50 & 50 & 60 & 83.3 & 83.3 & 3 No. $6 \mathrm{~mm} \phi$ & Straights & 0.15 & 65.3 & 4No. $6 \mathrm{~mm} \phi$ & Straights & $4 \mathrm{No} .6 \mathrm{~mm} \phi$ & Straights & $\begin{array}{c}\text { 6No. BFRP @ } \\
12.34 \mathrm{~mm}^{2}\end{array}$ \\
\hline $\mathrm{R} 2 / \mathrm{C} / 20$ & 1667 & 100 & 980 & 100 & 50 & 147 & 147 & 83.3 & 3 No. $6 \mathrm{~mm} \phi$ & Mesh & 0.15 & 65.3 & 4 No. $6 \mathrm{~mm} \phi$ & Mesh & 4 No. $6 \mathrm{~mm} \phi$ & Mesh & $\begin{array}{c}\text { 3No.CFRP @ } \\
19.65 \mathrm{~mm}^{2}\end{array}$ \\
\hline $\mathrm{R} 2 / \mathrm{B} / 20$ & 1667 & 100 & 980 & 100 & 50 & 147 & 147 & 83.3 & 3 No. $6 \mathrm{~mm} \phi$ & Straights & 0.15 & 65.3 & 4 No. $6 \mathrm{~mm} \phi$ & Straights & 4 No. $6 \mathrm{~mm} \phi$ & Straights & $\begin{array}{c}\text { 6No. BFRP @ } \\
12.34 \mathrm{~mm}^{2} \\
\end{array}$ \\
\hline $\mathrm{R} 2 / \mathrm{B} / 15$ & 1667 & 100 & 980 & 100 & 50 & 143 & 143 & 111.1 & 4 No. $6 \mathrm{~mm} \phi$ & Mesh & 0.15 & 93.1 & 4No. $6 \mathrm{~mm} \mathrm{\phi}$ & Mesh & 4No. $6 \mathrm{~mm} \phi$ & Mesh & $\begin{array}{c}\text { 8No. BFRP @ } \\
12.34 \mathrm{~mm}^{2}\end{array}$ \\
\hline
\end{tabular}




\subsubsection{Slab testing}

A typical slab test arrangement is illustrated in Figure 8. All slabs were supported on roller supports. For restrained samples, electrical resistance strain (ERS) gauges were attached to the upper and lower faces of each steel reinforcement bar within one restraint beam at mid-span. A central knife edge line load was applied in a three point loading arrangement using a spreader beam to each test slab, as shown in Figure 8. Prior to ultimate testing, all slabs were initially loaded twice up to the point of initial underside cracking, with a five minute rest period between each test load. This allowed test samples to recover sufficiently prior to the application of ultimate test loads. During ultimate loading, loads were increased in $0.2 \mathrm{kN}$ increments, under stroke control, by an accurately calibrated $600 \mathrm{kN}$ capacity hydraulic actuator until failure occurred. Ultimate capacity was identified as the point beyond which maximum load could no longer be sustained. Table 6 summarises these values and shows that failure loads typically increased with the addition of FRP strengthening and increasing restraint.

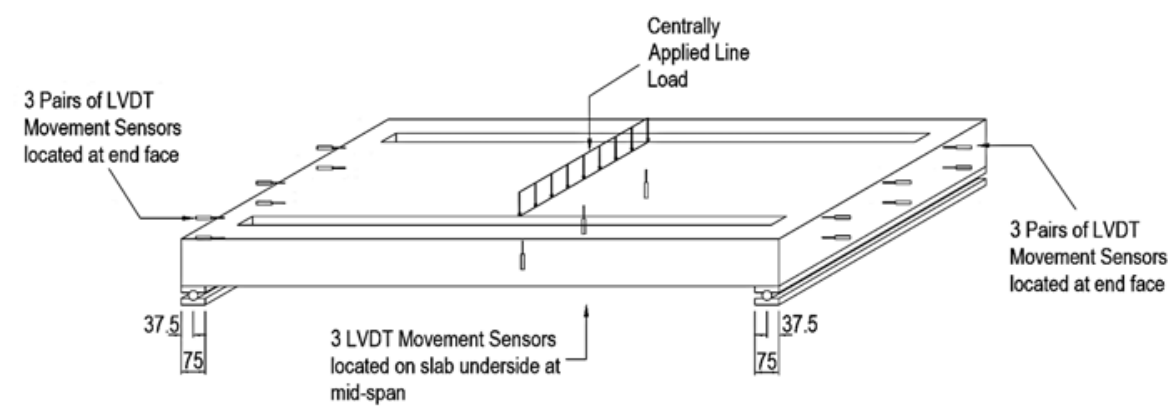

Figure 8: Typical test slab setup (all dimensions in millimetres)

Table 6: Summary of test slab failure loads

\begin{tabular}{|c|c|c|c|}
\hline $\begin{array}{l}\text { Slab } \\
\text { Code }\end{array}$ & $\begin{array}{l}\text { Experimental Failure Load, } P_{t} \\
\qquad(k N)\end{array}$ & $\begin{array}{l}\text { Slab } \\
\text { Code }\end{array}$ & $\begin{array}{l}\text { Experimental Failure Load, } P_{t} \\
(k N)\end{array}$ \\
\hline$S / N / 20$ & 7.02 & R4/N/15 & 34.54 \\
\hline S/N/15 & 12.25 & $\mathrm{R} 1 / \mathrm{C} / 20$ & 17.52 \\
\hline$S / C / 20$ & 17.48 & $\mathrm{R} 1 / \mathrm{B} / 20$ & 20.74 \\
\hline$S / B / 20$ & 20.70 & $\mathrm{R} 0.5 / \mathrm{C} / 20$ & 15.77 \\
\hline$S / B / 15$ & 33.73 & $\mathrm{R} 0.5 / \mathrm{B} / 20$ & 19.05 \\
\hline$R 1 / N / 20$ & 10.43 & $\mathrm{R} 2 / \mathrm{C} / 20$ & 22.45 \\
\hline $\mathrm{R} 0.5 / \mathrm{N} / 20$ & 7.92 & $\mathrm{R} 2 / \mathrm{B} / 20$ & 24.23 \\
\hline $\mathrm{R} 2 / \mathrm{N} / 20$ & 16.11 & R2/B/15 & 46.26 \\
\hline $\mathrm{R} 2 / \mathrm{N} / 15$ & 27.51 & & \\
\hline
\end{tabular}




\subsubsection{Determination of experimental in-plane restraint stiffness}

In-plane restraint stiffness is the ratio between the compressive force resisting a slab from spreading and the distance through which spreading occurs. The evaluation of the restraint stiffness, $k_{r}$, in experimental slab specimens due to the presence of inplane restraint beams was therefore based upon restraint forces established from an analysis of electrical resistance strain (ERS) gauge readings attached to restraint beam reinforcement bars and longitudinal slab movements recorded using Linear Variable Differential Transformers (LVDTs), as shown in Figure 8.

\subsubsection{Calculating the strength of an in-plane restrained slab}

The determination of restrained slab capacity, based upon experimental restraint data, was carried out using the QUB arching theory approach initially developed by Rankin [19], explained in detail by Tharmarajah [26] and adopted by Tharmarajah et al. [57]. Figure 3 illustrates the iterative process involved in establishing slab capacity using this method. An excel spreadsheet was used to automate these calculations and, generally, only a few iterations were required to obtain stable estimations.

As a worked example, slab $\mathrm{R} 2 / \mathrm{C} / 20$ shall now be evaluated in accordance with the following material properties:

- Yield strength of steel reinforcement, $f_{y k}=549.30 \mathrm{~N} / \mathrm{mm}^{2}$

- Young's Modulus for Steel Reinforcement, $E_{s}=201.94 \mathrm{kN} / \mathrm{mm}^{2}$

- Compressive strength of concrete, $f_{c k, c u b e}=43.12 \mathrm{~N} / \mathrm{mm}^{2}$

$k_{r}=232.41 \mathrm{kN} / \mathrm{mm}$

Calculations to establish the simply supported slab capacity for slab $\mathrm{R} 2 / \mathrm{C} / 20$ based on Eurocode and $\mathrm{ACl}$ codes are not given here, as they can be easily determined by following these codes. However, these show that their mid-span point load capacity is $16.64 \mathrm{kN}$ and $16.46 \mathrm{kN}$ by EC2 and $\mathrm{ACl}$ respectively. This shall now be compared with the determination of the slab capacity using arching theory incorporating the experimentally determined restraint of $232.41 \mathrm{kN} / \mathrm{mm}$. 


\section{Step 1}

\section{Stiffness parameters}

Restraint stiffness $\quad k_{r}=232.41 \mathrm{kN} / \mathrm{mm}$

\section{Bending capacity}

Proportional depth of stress block factor:

$\beta=1-0.003 f_{\text {ck,cube }}$ but $\leq 0.9$

$\beta=1-0.003 \times 43.12=0.871$

Depth of neutral axis: $x=\frac{A_{S} f_{y k}+A_{F R P} f_{F R P, k}}{0.67 f_{c k, c u b e} \beta b}=\frac{84.82 \times 549.30+58.95 \times 990.42}{0.67 \times 43.12 \times 0.871 \times 680}=6.14 \mathrm{~mm}$

Moment capacity due to bending:

$$
\begin{aligned}
& M_{b}=\left(A_{s} f_{y k}\right)\left[d-\frac{\beta x}{2}\right]+\left(A_{F R P} f_{F R P, k}\right)\left[d_{F R P}-\frac{\beta x}{2}\right] \\
& M_{b}=(84.82 \times 549.30)\left[65.30-\frac{0.871 \times 6.14}{2}\right]+(58.95 \times 990.42)\left[79.48-\frac{0.871 \times 6.14}{2}\right] \\
& M_{b}=7.40 \mathrm{kNm}
\end{aligned}
$$

Load corresponding to bending moment capacity:

$P_{b}=\frac{4 M_{b}}{L}=\frac{4 \times 7.40}{1.792}=16.52 k N$

\section{Step 2}

\section{Arching section}

Depth available for arching: $d_{1}=\frac{h-2 \times \beta}{2}=\frac{83.3-2 \times 6.14 \times 0.871}{2}=36.31 \mathrm{~mm}$

\section{Affine strip}

Area of concrete due to arching (assume $\alpha=1$ for first iteration):

$A=\alpha b d_{1}=1 \times 680 \times 36.31=20861.45 \mathrm{~mm}^{2}$

Equivalent rigid half arch span:

$$
L_{r}=L_{e}\left[\frac{E_{c} A}{k_{r} L_{e}}+1\right]^{1 / 3}=\left(\frac{1792}{2}\right) \times\left[\frac{4230 \times \sqrt{43.12} \times 25195}{\left(232.41 \times 10^{3}\right) \times\left(\frac{1792}{2}\right)}+1\right]^{1 / 3}=1396.06 \mathrm{~mm}
$$




\section{Arching parameters}

Ultimate compressive strain in concrete:

$\varepsilon_{u}=0.0043-\left[f_{c k, c u b e}-60\right] \times\left(2.5 \times 10^{-5}\right)$ but $\leq 0.0043$

$\varepsilon_{u}=0.0043-[43.12-60] \times\left(2.5 \times 10^{-5}\right)=0.0047>0.0043$

Therefore let $\varepsilon_{u}=0.0043$

Concrete plastic strain:

$\varepsilon_{c}=2 \varepsilon_{u}(1-\beta)=2 \times 0.0043 \times(1-0.871)=0.0011$

McDowell's non-dimensional geometry and material factor:

$R=\frac{\varepsilon_{c} L_{r}^{2}}{4 d_{1}^{2}}=\frac{0.0011 \times 1396.06^{2}}{4 \times 36.31^{2}}=0.411$

\section{Deformation}

$R>0.26 \quad$ Therefore: $\quad u=0.31$

\section{Contact depth}

$\alpha=1-\frac{u}{2}=1-\frac{0.31}{2}=0.845$

Therefore, the refined area of concrete in arching becomes:

$A=\alpha b d_{1}=0.845 \times 680 \times 36.31=20861.45 \mathrm{~mm}^{2}$

Iterations within step 2 results in the following values:

$u=0.31$

$\varepsilon_{u}=0.00430$

$\alpha=0.845$

$\varepsilon_{c}=0.00111$

$A=20861.45 \mathrm{~mm}^{2}$

$R=0.411$

$L_{r}=1396.06 \mathrm{~mm}$ 


\section{Step 3}

\section{Arching capacity}

$R>0.26 \quad$ Therefore, moment ratio:

$M_{r}=\frac{0.3615}{R}=\frac{0.3615}{0.411}=0.8791$

Equivalent rigid arching moment of resistance:

$M_{a r}=0.168 b f_{c k, c u b e} d_{1}^{2} M_{r}\left(\frac{L_{e}}{L_{r}}\right)$

$M_{a r}=0.168 \times 680 \times 43.12 \times 36.31^{2} \times 0.8791 \times\left(\frac{(1792 / 2)}{1396.06}\right)=3.663 \mathrm{kNm}$

Elastic arching moment of resistance:

$M_{a}=M_{a r}\left(\frac{L_{e}}{L_{r}}\right)=3.663 \times\left(\frac{(1792 / 2)}{1396.06}\right)=2.351 \mathrm{kNm}$

Load corresponding to arching capacity:

$P_{a}=\frac{4 M_{a}}{L}=\frac{4 \times 2.351}{1.792}=5.25 k N$

\section{Ultimate capacity}

$P_{p}=P_{a}+P_{b}=5.25+16.52=21.77 k N$

Thus, by considering the in-plane restraint inherent within this slab, and the contribution due to FRP strengthening, a capacity increase of approximately $31.5 \%$ was established in comparison with Eurocode and $\mathrm{ACl}$ predictions. An excel spreadsheet was used to automate the iterative calculations above. Generally, only a few iterations were required to obtain stable estimations, but the Microsoft Excel macro was set to allow up to 100 iterations.

Predicted capacities, using QUB arching theory with experimental restraint stiffness values are given in Table 7 alongside comparisons with capacity estimates obtained using Eurocode 2 [28] and the American Concrete Institute [29] [58]. 
Table 7: Comparison of predicted and experimental capacities for test slabs

\begin{tabular}{|c|c|c|c|c|c|c|c|}
\hline Slab Code & $\begin{array}{c}\text { EC2 } \\
\text { Predicted } \\
\text { Load, } \\
P_{\mathrm{p}, \mathrm{EC2}}(\mathrm{kN})\end{array}$ & $\begin{array}{c}\text { ACI Predicted } \\
\text { Load, } \mathrm{P}_{\mathrm{p}, \mathrm{ACl}} \\
(\mathrm{kN})\end{array}$ & $\begin{array}{c}\text { Arching } \\
\text { Theory } \\
\text { Predicted } \\
\text { Load, } \mathrm{P}_{\mathrm{p} \text {,arching }} \\
(\mathrm{kN})\end{array}$ & $\begin{array}{l}\text { Experimenta } \\
\text { I Failure } \\
\text { Load, } \mathbf{P}_{\mathrm{t}}(\mathbf{k N})\end{array}$ & $\begin{array}{l}P_{p} / P_{t} \\
(E C 2)\end{array}$ & $\begin{array}{l}\mathrm{P}_{\mathrm{p}} / \mathrm{P}_{\mathrm{t}} \\
(\mathrm{ACl})\end{array}$ & $\begin{array}{c}P_{p} / P_{t} \\
\text { (Arching) }\end{array}$ \\
\hline$S / N / 20$ & 6.09 & 6.08 & 6.07 & 7.02 & 0.87 & 0.87 & 0.87 \\
\hline$S / N / 15$ & 11.61 & 11.60 & 11.58 & 12.25 & 0.95 & 0.95 & 0.95 \\
\hline $\mathrm{S} / \mathrm{C} / 20$ & 16.65 & 16.47 & 16.54 & 17.48 & 0.95 & 0.94 & 0.95 \\
\hline $\mathrm{S} / \mathrm{B} / 20$ & 20.77 & 20.69 & 20.64 & 20.70 & 1.00 & 1.00 & 1.00 \\
\hline S/B/15 & 37.61 & 37.51 & 37.33 & 33.73 & 1.11 & 1.11 & 1.11 \\
\hline $\mathrm{R} 1 / \mathrm{N} / 20$ & 6.09 & 6.08 & 13.09 & 10.43 & 0.58 & 0.58 & 1.26 \\
\hline$R 0.5 / N / 20$ & 6.09 & 6.09 & 7.48 & 7.92 & 0.77 & 0.77 & 0.94 \\
\hline $\mathrm{R} 2 / \mathrm{N} / 20$ & 6.09 & 6.08 & 17.11 & 16.11 & 0.38 & 0.38 & 1.06 \\
\hline $\mathrm{R} 2 / \mathrm{N} / 15$ & 11.59 & 11.57 & 26.85 & 27.51 & 0.42 & 0.42 & 0.98 \\
\hline R4/N/15 & 12.71 & 12.69 & 39.84 & 34.54 & 0.37 & 0.37 & 1.15 \\
\hline $\mathrm{R} 1 / \mathrm{C} / 20$ & 16.64 & 16.46 & 20.40 & 17.52 & 0.95 & 0.94 & 1.16 \\
\hline $\mathrm{R} 1 / \mathrm{B} / 20$ & 21.25 & 21.19 & 27.81 & 20.74 & 1.02 & 1.02 & 1.34 \\
\hline $\mathrm{R} 0.5 / \mathrm{C} / 20$ & 16.74 & 16.52 & 17.67 & 15.77 & 1.06 & 1.05 & 1.12 \\
\hline $\mathrm{R} 0.5 / \mathrm{B} / 20$ & 20.69 & 20.63 & 20.87 & 19.05 & 1.09 & 1.08 & 1.10 \\
\hline $\mathrm{R} 2 / \mathrm{C} / 20$ & 16.64 & 16.46 & 21.77 & 22.45 & 0.74 & 0.73 & 0.97 \\
\hline $\mathrm{R} 2 / \mathrm{B} / 20$ & 20.69 & 20.63 & 27.60 & 24.23 & 0.85 & 0.85 & 1.14 \\
\hline $\mathrm{R} 2 / \mathrm{B} / 15$ & 38.63 & 37.56 & 46.11 & 46.26 & 0.84 & 0.81 & 1.00 \\
\hline \multirow{2}{*}{\multicolumn{5}{|c|}{$\begin{array}{r}\text { Average }= \\
\text { Standard Deviation }=\end{array}$}} & 0.82 & 0.82 & 1.06 \\
\hline & & & & & 0.25 & 0.24 & 0.13 \\
\hline \multicolumn{5}{|c|}{ Coefficient of variation $=$} & 0.06 & 0.06 & 0.01 \\
\hline
\end{tabular}

\section{NLFEA modelling}

\subsection{Analysis types}

The London University Structural Analysis System (LUSAS) was chosen to carry out NLFEA investigations. This software offered a range of analysis types of which 'general structural analysis' was selected to carry out investigations of in-plane restrained concrete slabs. Further details of other analysis options can be found in the LUSAS Modeller Reference Manual [59]. By default, LUSAS was set to carry out a static linear elastic analysis, as this was considered by the application's developers to be the most prevalent analysis type required by practising design engineers. Typically, this applies to situations where deformations are below those exceeding material elastic limits. However, due to the relatively large deformations observed in laboratory samples, nonlinear analyses were considered necessary for these investigations. This therefore involved modifying the default settings of the LUSAS 
general structural analysis. Hence, material nonlinearity of concrete, steel, BFRP and CFRP were included, with values based upon experimental test data.

\subsection{Model construction}

3D models were constructed by initially defining, on plan, the lines which made up the outer slab geometry along with the locations of internal reinforcement and outer regions of loading and support. LUSAS then had the facility to permit the formation of connecting lines into surfaces and to then sweep these surfaces into volumes. Reinforcement and FRP bars were selected as individual groups to which mesh and material properties could then be assigned. Similarly, mesh and material properties were assigned to $3 \mathrm{D}$ volume (i.e. concrete) regions. In each case, ultimate slab capacity was identified as the point where midspan reinforcement/FRP reached yield/rupture stress accompanied by a sharp increase in midspan vertical deflections, as observed in experimental tests.

\subsection{Supports}

Global axes were selected as shown in Figure 9. As each experimental test slab was supported by a roller, as detailed in Figure 8 , these supports were modelled as being free to rotate about all axes but translationally fixed in $\mathrm{X}$ - and $\mathrm{Y}$-directions. An additional Z-direction translational restraint was also included at one end in cases where full slabs were initially modelled. However, in all other cases, where symmetry allowed smaller slab regions to be modelled, centreline and midspan restraints were used, as illustrated in Figure 9.

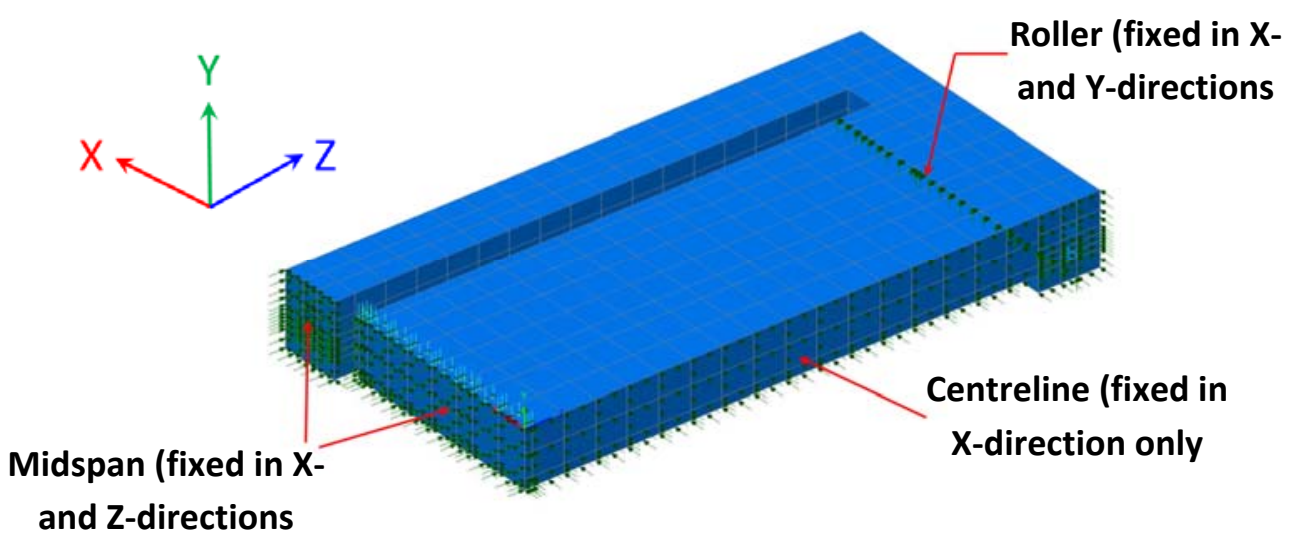

Figure 9: Quarter 3D model illustrating translational restraints (R4/N/15) 


\subsection{Loading}

Pressures, known within LUSAS as 'face loads', were initially applied under load control over $25 \mathrm{~mm}$ wide regions at mid-span, representative of experimental procedures. Unfortunately, however, applying monotonic loading in this way resulted in load-deflection output which was initially over stiff and which displayed excessive peak loads at low deflections, as shown in Figure 10. However, these characteristics were not reflective of experimental behaviour. To address this problem, deflection control was employed with repeated loading to mimic the deflections encountered during the initial cracking and ultimate test phases of experimental testing. This removed initial over-stiffness and excessive peaks in all slab models, as shown in Figure 10.

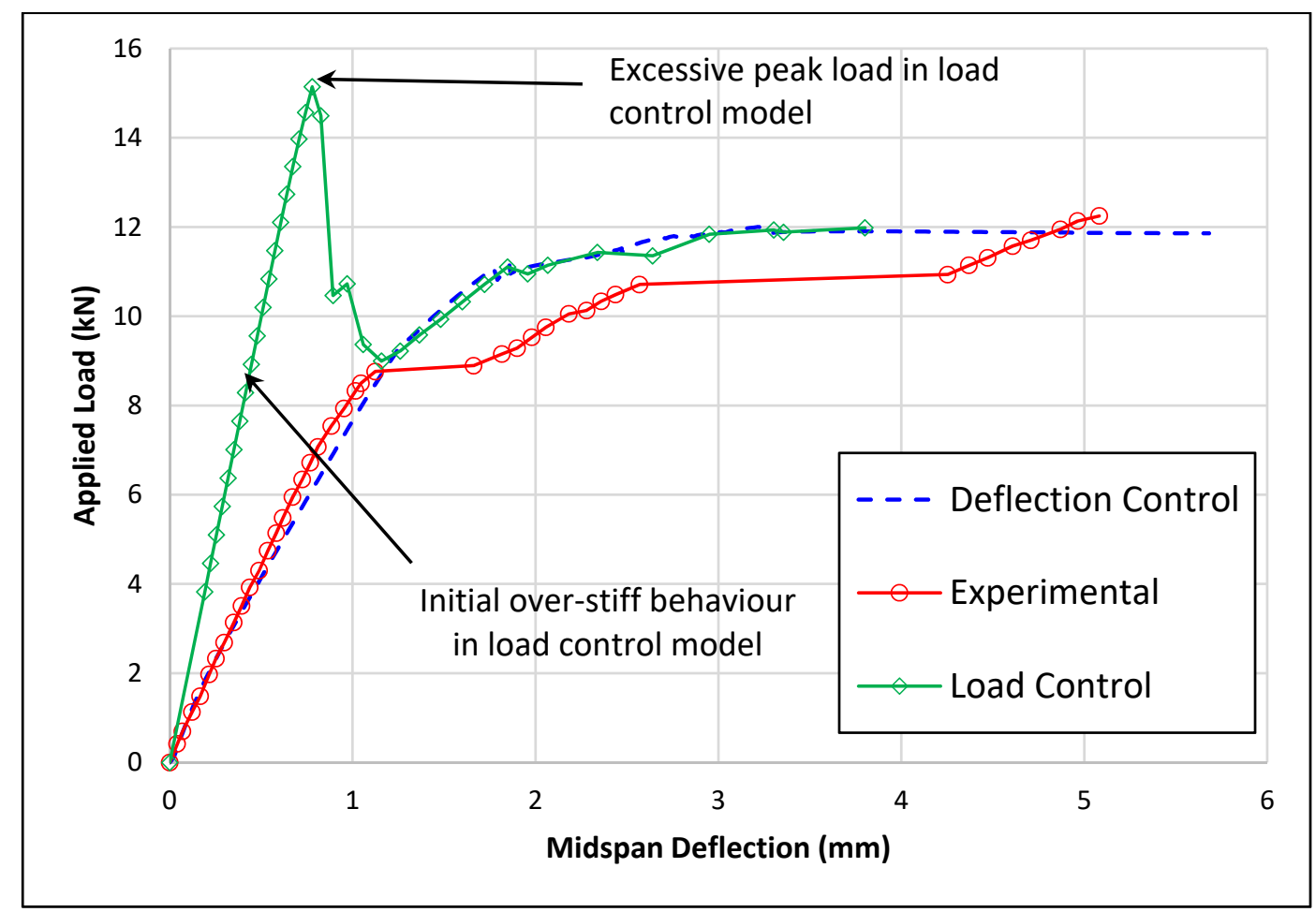

Figure 10: Comparison between load and deflection control output for slab $\mathrm{S} / \mathrm{N} / 15$

\subsection{Element type}

Each LUSAS model consisted of lines, bars and volumes; each requiring a different element type. In each case the number of nodal divisions or the distance between nodes could be defined. These are outlined in the following subsections. 


\subsubsection{Lines}

Lines defined the outer geometry and internal volumetric subdivisions within each 3D model, although they did not require the allocation of attributes such as material properties or cross sectional areas. Hence, NULL elements were used to produce a $2 \mathrm{D}$ null line mesh in each case.

\subsubsection{Volumes}

3D models were used due to the experimental slabs' relative geometric simplicity and the available speeds of analysis. To construct each model, 3D solid continuum elements were selected to model concrete volumes. Of those available, 20 noded HX20 elements were identified [60] as being suitable for use in modelling reinforced concrete. HX20 elements permitted regular mesh patterns and could accommodate boundary curvatures and face loading.

\subsubsection{Bars}

Within LUSAS, bar elements could be used to model plane and space truss structures and steel reinforcement. These elements only consider axial forces and LUSAS [59] recommended using bar elements to model reinforcement within 3D reinforced concrete models; with the exact element choice dependent upon selecting the number of bar element nodes (i.e. 2 or 3 ) and the interpolation order (i.e. two noded linear or three noded quadratic). Both two- and three-noded bar elements were suitable for modelling reinforcement with continuum elements and, as HX20 elements were selected for modelling concrete volumes, three-noded BRS3 bar elements were chosen to model steel reinforcement and FRP bars.

\subsection{LUSAS material models}

\subsubsection{Concrete Models}

LUSAS offered two concrete material models: 'multi crack concrete model' (model 94), and the 'smoothed multi crack concrete model' (model 102). Model 102 was described as being similar to model 94 but with more rapid convergence and robustness [59]. In each case, softening could be controlled via fixed softening curves in compression and tension, as shown in Figure 11, or by a fracture energy controlled softening curve dependent upon element size. However, LUSAS [59] recommended using a fixed softening curve in reinforced concrete models and this approach was therefore adopted. For each material model, similar input parameters were required. 
However, as model 102 was the latest evolution of LUSAS' concrete models, offering faster analysis times and greater accuracy, this was selected for use in these NLFEA investigations. The following values, identified in Figure 11, were used in these analyses:

- $\mathrm{f}_{\mathrm{c}}=$ Experimental compressive strength values (with a target value of 40 $\mathrm{N} / \mathrm{mm}^{2}$ )

- $\mathrm{f}_{\mathrm{t}}=$ Experimental cylinder splitting test values (also further investigations with code recommended values)

- $\varepsilon_{\mathrm{c}}=$ ratio of experimental $\mathrm{f}_{\mathrm{c}}$ to $\mathrm{E}_{\mathrm{c}}$ (determined with the use of equation (21))

- $\varepsilon_{0}=2.5 \times 10^{-3}($ Recommended value [61])

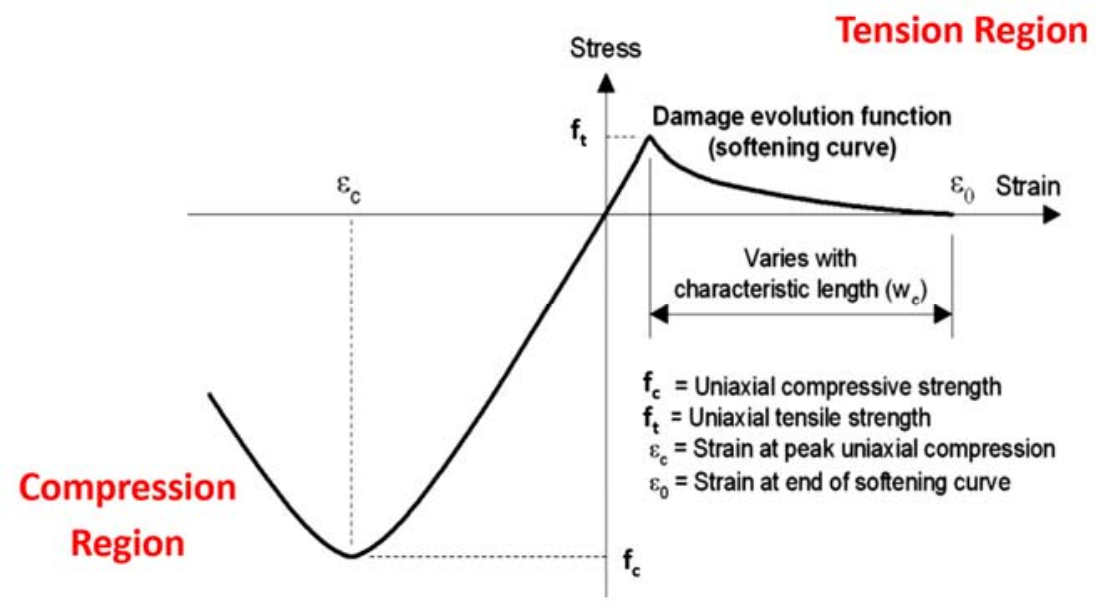

Figure 11: Stress / strain curve for multi crack concrete models [59]

\subsubsection{NLFEA reinforcement and FRP modelling}

LUSAS permitted steel reinforcement and FRP properties to be defined in two parts. Firstly, Young's modulus and Poisson's ratio were defined as 'elastic' parameters. Young's modulus values were based on experimental test data, as given in Tables 2 and 3 and assumed Poisson's ratio values of 0.3 and 0.15 were used for steel reinforcement and FRP bars respectively. Secondly, 'plastic' properties were selected with a Von Mises stress potential and total strain hardening; where post elastic stresses and corresponding strains, based on experimental data, were then defined in tabular format. 


\subsection{Comparison of quarter, half and full slab models}

Due to midspan and longitudinal symmetry, the potential to model one quarter of each slab offered significant reductions in analysis time, if reflective of full slab model behaviour. Therefore, a representative slab with regular restraint and BFRP strengthening $(\mathrm{R} 1 / \mathrm{B} / 20)$ was chosen to investigate the differences between full, half and quarter 3D NLFEA slab models, as shown in Figure 12.
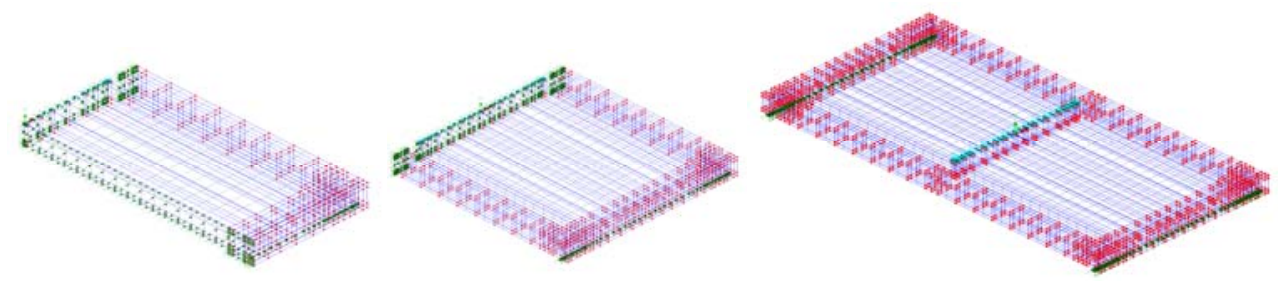

Figure 12: Full, half and quarter 3D models (R1/B/20)

In each case, identical concrete, steel and BFRP properties, based upon laboratory test results, were used. A recommended value for the ultimate tensile strain in concrete, $\varepsilon_{U L T}$, of $2.5 \times 10^{-3}$ was selected for concrete [61], along with a mesh size of $50 \mathrm{~mm}$. Load-deflection plots were generated for each case, as illustrated in Figure 13 , and show that results from full, half and quarter slabs were nearly indistinguishable from each other, although deflections in each instance were lower than in experiments. Ultimate load predictions for each LUSAS model are also outlined in Table 8. Given the close similarity between load-deflection output and ultimate capacity predictions between each numerical model, quarter models were therefore selected for modelling all subsequent slabs due to faster analysis times.

Table 8: Ultimate capacity predictions using 3D solid NLFEA models with similar material properties (R1/B/20)

\begin{tabular}{|c|c|}
\hline Slab Model Type & 3D Solid Capacity (kN) \\
\hline Full & 21.89 \\
\hline Half & 21.89 \\
\hline Quarter & 21.62 \\
\hline
\end{tabular}




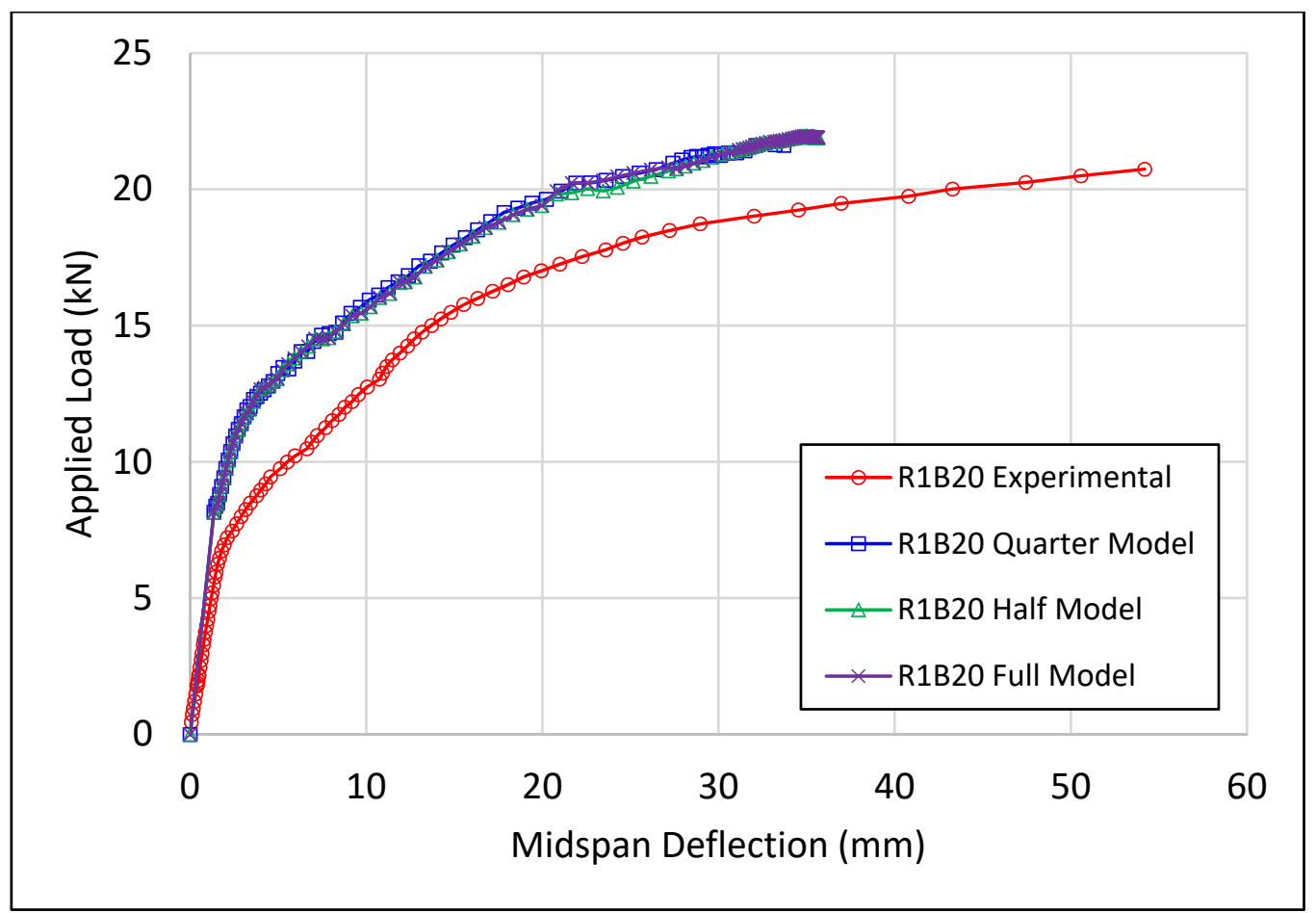

Figure 13: Comparison of load - deflection performance of full, half and quarter 3D models (R1/B/20)

\subsection{Mesh size}

Finite element mesh density has an effect on analysis time and on the accuracy of output results. Typically, the higher the mesh density (i.e. the smaller the finite elements used) the more accurate the results. It has also been reported [62] that smaller elements have the capability to eliminate unrealistically low strength predictions due to stress concentration effects whilst large elements reduce the need to modify constitutive models to avoid overestimating energy dissipation capacity. It was therefore necessary to examine the effect of mesh size variations to establish a mesh size for use in subsequent investigations. Slabs S/N/20, S/N/15, R2/N/15 and $\mathrm{R} 2 / \mathrm{C} / 20$ were therefore chosen as being representative of the overall cohort of test specimens.

Mesh sizes of $15 \mathrm{~mm}, 30 \mathrm{~mm}, 40 \mathrm{~mm}, 50 \mathrm{~mm}$ and $100 \mathrm{~mm}$ were considered. A further mesh size of $10 \mathrm{~mm}$ was also initially considered, although this led to unacceptably long analysis times (in excess of 24 hours for simply supported slabs) and, in several cases, resulted in insufficient memory errors resulting in their omission from further investigation. In each case, laboratory tested values for steel reinforcement, FRP and concrete were used along with $\varepsilon_{\cup L T}=2.5 \times 10^{-3}[61]$. 
Load-deflection output, illustrated in Figures 14(a) - 14(d), showed relatively little variation in behaviour between the mesh sizes considered, particularly where FRP strengthening was omitted. Predicted-to-test value $\left(P_{p} / P_{t}\right)$ ratios for each mesh size; illustrated in Figure 15 and summarised in Table 9; showed that close agreement with experiments was achieved $\left(P_{p} / P_{t}=0.96\right)$ with a $40 \mathrm{~mm}$ mesh which did not change, on average, as mesh sizes reduced. However, sample R2/C/20 displayed a drop in predicted capacity with a mesh size of $15 \mathrm{~mm}$ suggesting that capacity estimates were likely to be underestimated in cases involving FRP strengthening and low mesh size. It is considered likely that this behaviour was due to the nature of cohesion between FRP bars and concrete in NLFEA models with high mesh density, although a thorough investigation of this behaviour is considered to be beyond the scope of the current investigations. Furthermore, analysis times increased considerably; in some cases by a factor of 30 ; with smaller mesh sizes. Hence, a $40 \mathrm{~mm}$ mesh was selected for all subsequent LUSAS models.

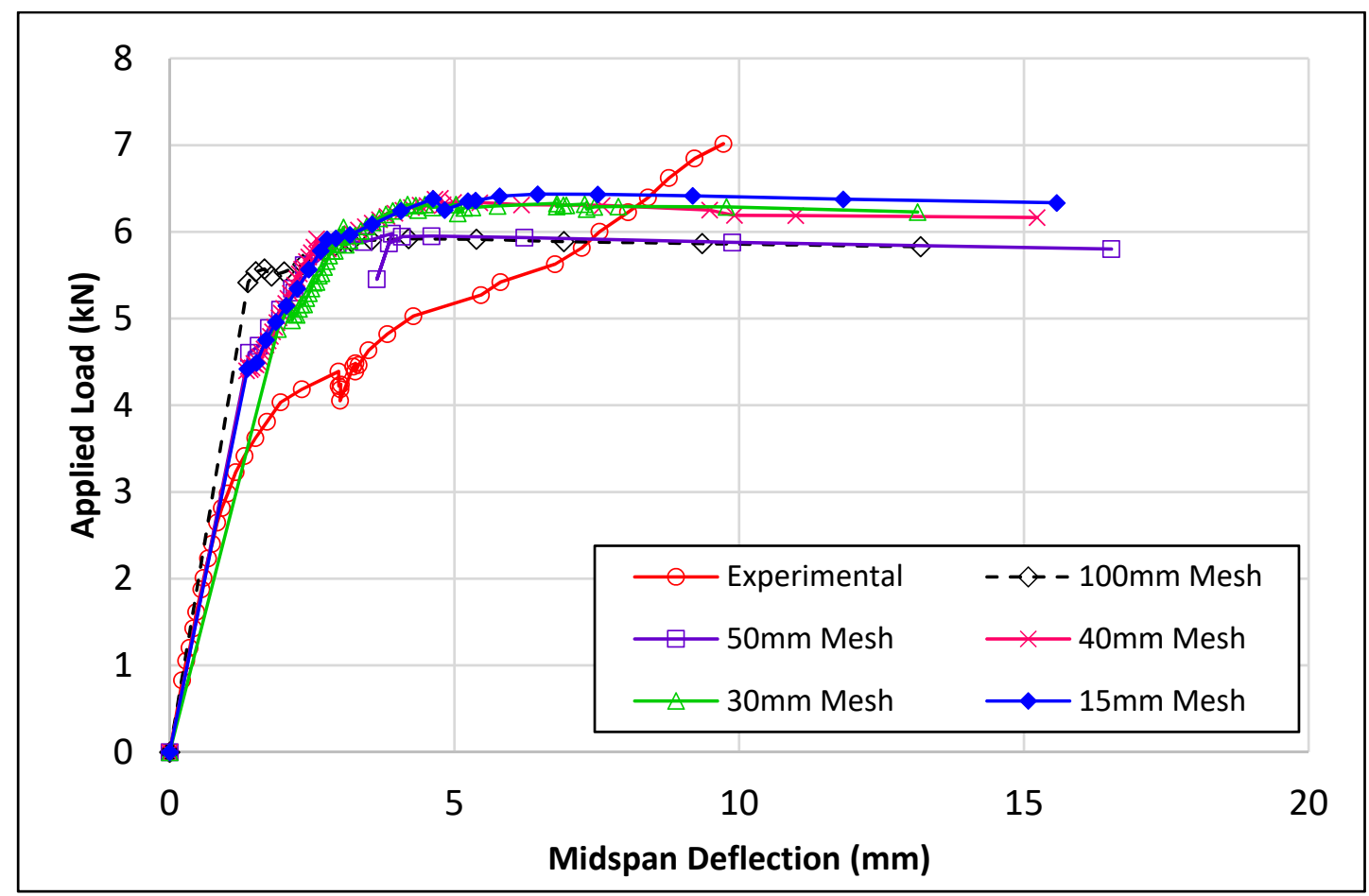

Figure 14(a): Load - deflection behaviour of 3D S/N/20 models with varying mesh sizes 


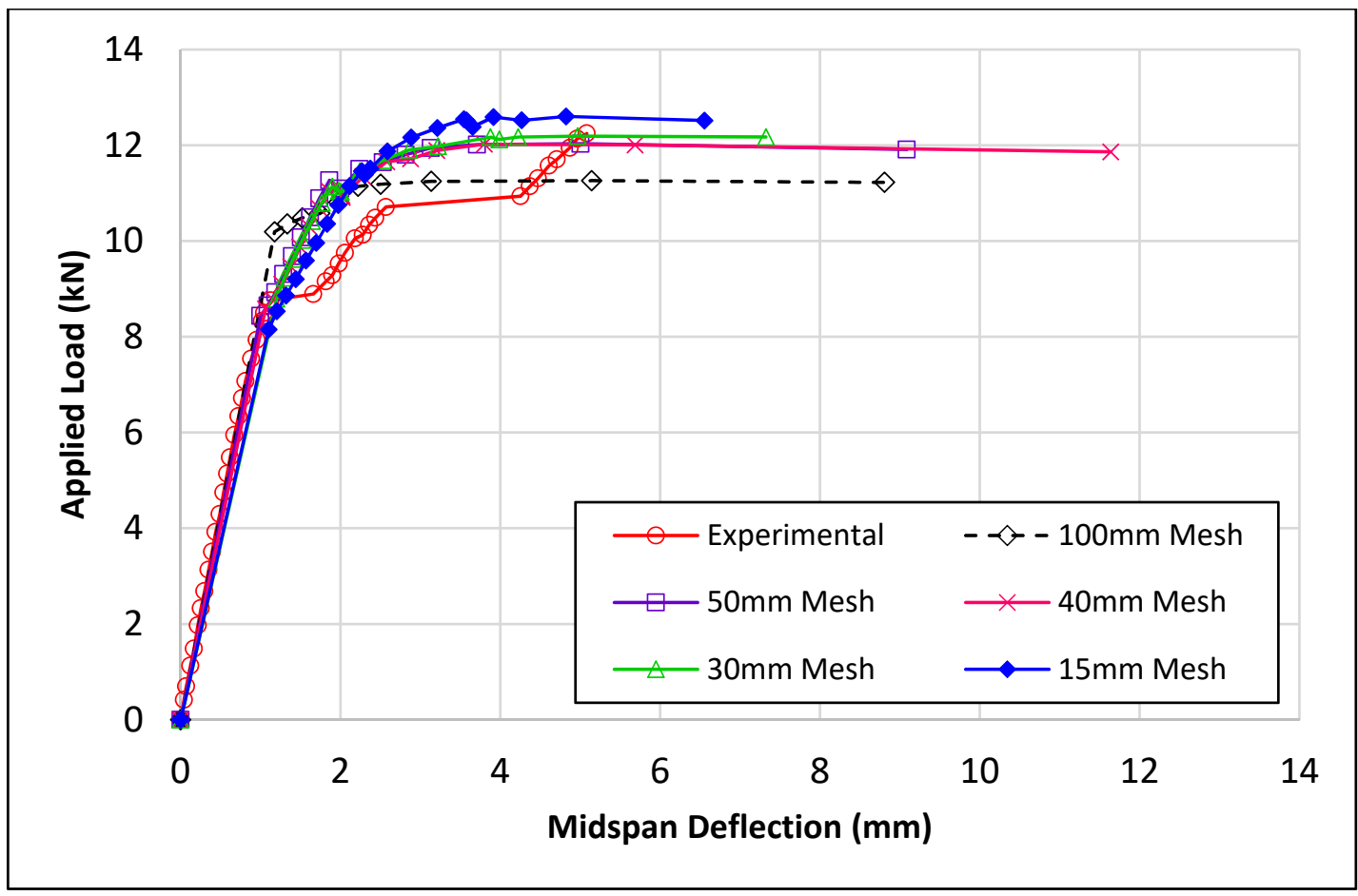

Figure 14(b): Load - deflection behaviour of 3D S/N/15 models with varying mesh sizes

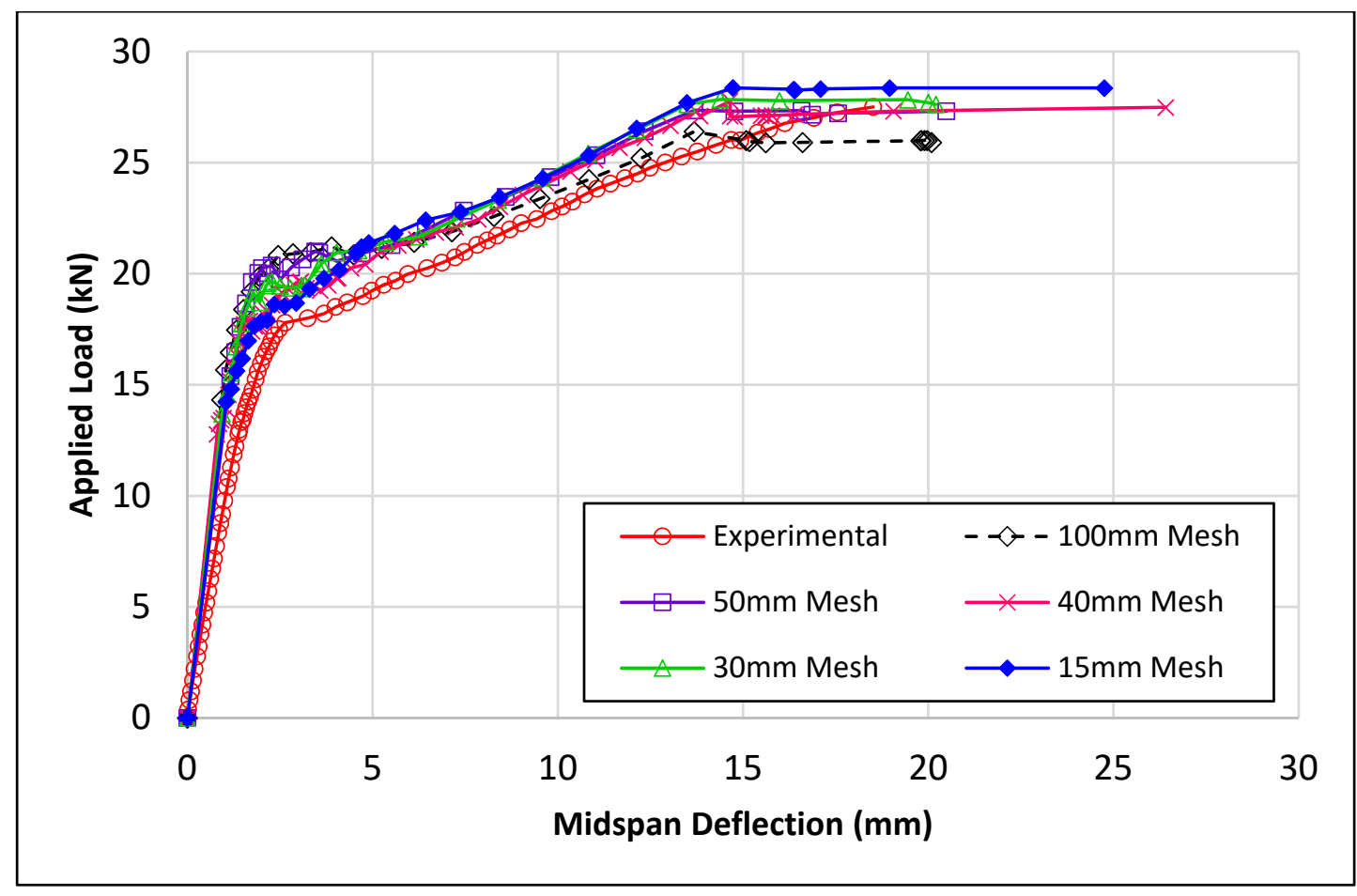

Figure 14(c): Load - deflection behaviour of 3D R2/N/15 models with varying mesh sizes 


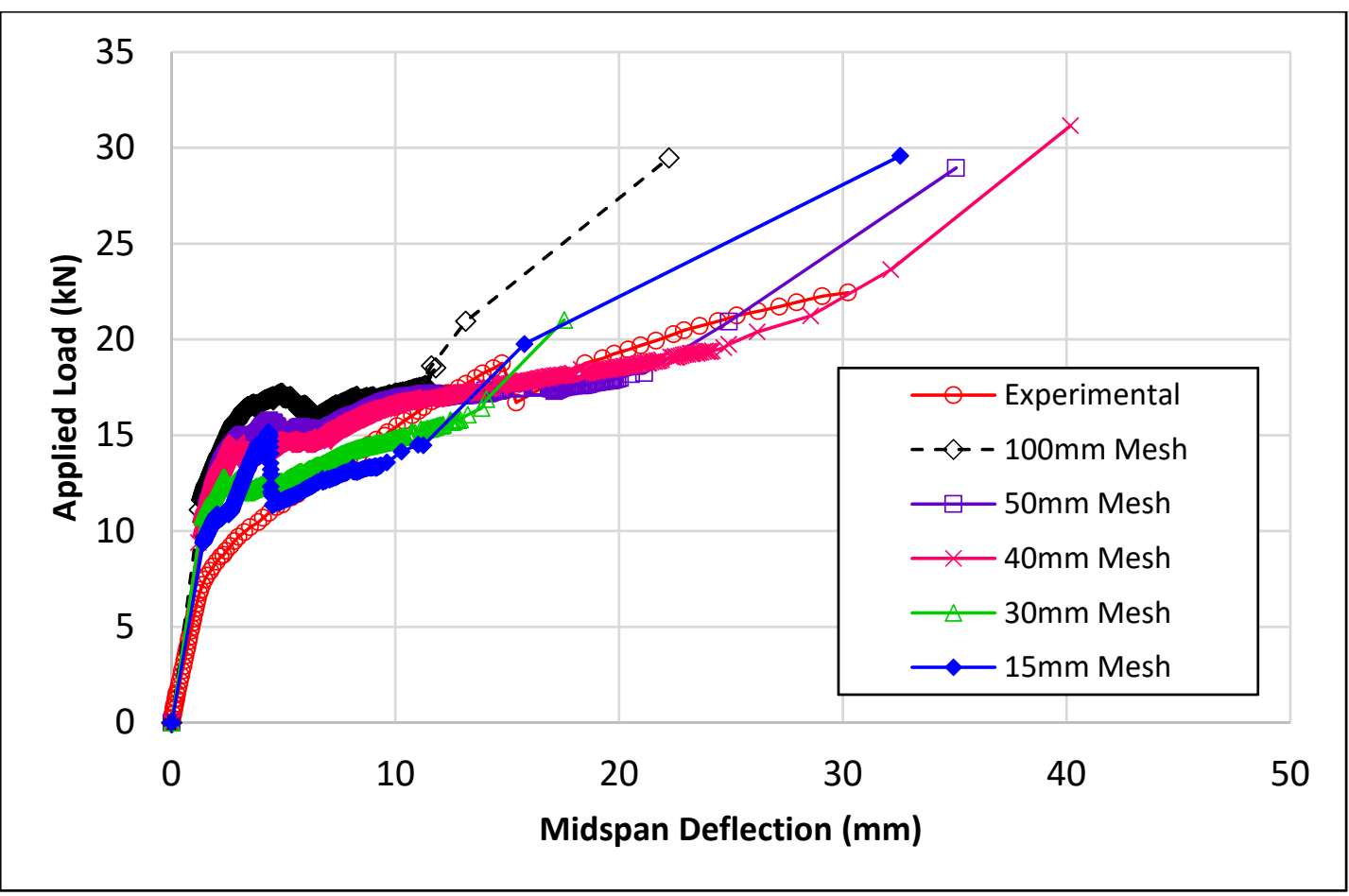

Figure 14(d): Load - deflection behaviour of 3D R2/C/20 models with varying mesh sizes

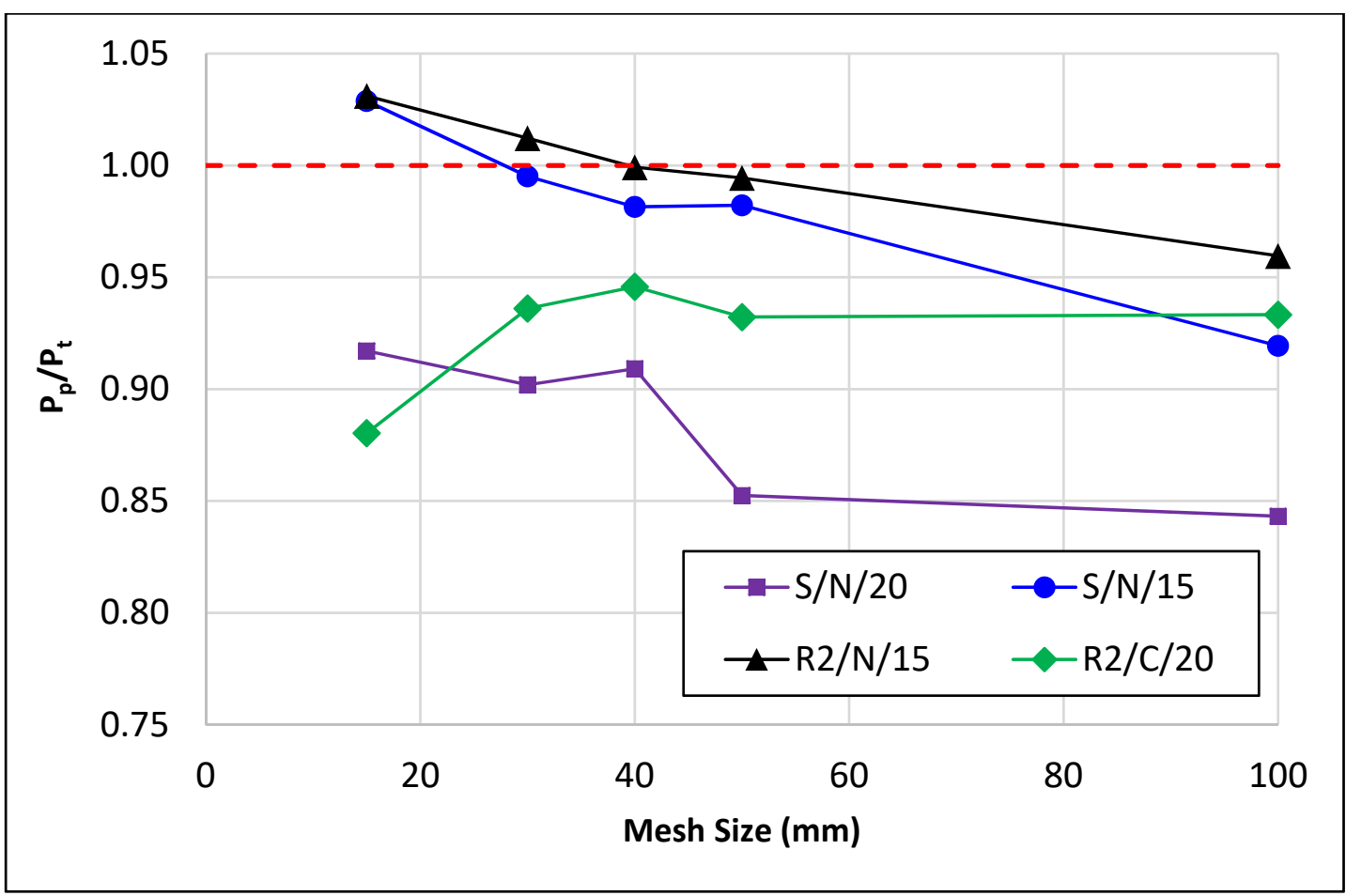

Figure 15: Variation of mesh size with $P_{p} / P_{t}$ ratio for series of sample slabs featured in the research 
Table 9: Summary of NLFEA predicted and experimental capacities for representative slabs with varying mesh size

\begin{tabular}{|c|c|c|c|c|}
\hline Slab Code & $\begin{array}{l}\text { Mesh Size } \\
(\mathrm{mm})\end{array}$ & $\begin{array}{c}\text { Experimental Failure } \\
\text { Load }(k N)\end{array}$ & $\begin{array}{l}\text { LUSAS Predicted } \\
\text { Failure Load (kN) }\end{array}$ & $\mathbf{P}_{\mathrm{p}} / \mathbf{P}_{\mathrm{t}}$ \\
\hline $\mathrm{S} / \mathrm{N} / 20$ & 100 & 7.02 & 5.92 & 0.84 \\
\hline $\mathrm{S} / \mathrm{N} / 15$ & 100 & 12.25 & 11.26 & 0.92 \\
\hline $\mathrm{R} 2 / \mathrm{N} / 15$ & 100 & 27.51 & 26.40 & 0.96 \\
\hline $\mathrm{R} 2 / \mathrm{C} / 20$ & 100 & 22.45 & 20.95 & 0.93 \\
\hline & & & Average $P_{p} / P_{t}=$ & 0.91 \\
\hline
\end{tabular}

\begin{tabular}{|c||c|c|c|c|}
\hline $\mathrm{S} / \mathrm{N} / 20$ & 50 & 7.02 & 5.98 & 0.85 \\
\hline $\mathrm{S} / \mathrm{N} / 15$ & 50 & 12.25 & 12.03 & 0.98 \\
\hline $\mathrm{R} 2 / \mathrm{N} / 15$ & 50 & 27.51 & 27.36 & 0.99 \\
\hline $\mathrm{R} 2 / \mathrm{C} / 20$ & 50 & 22.45 & 20.93 & 0.93 \\
\hline \multicolumn{1}{l}{} & \multicolumn{3}{|c|}{ Average $\mathbf{P}_{\mathrm{p}} / \mathbf{P}_{\mathbf{t}}=$} & $\mathbf{0 . 9 4}$ \\
\hline
\end{tabular}

\begin{tabular}{|c|c|c|c|c|}
\hline $\mathrm{S} / \mathrm{N} / 20$ & 40 & 7.02 & 6.38 & 0.91 \\
\hline $\mathrm{S} / \mathrm{N} / 15$ & 40 & 12.25 & 12.02 & 0.98 \\
\hline $\mathrm{R} 2 / \mathrm{N} / 15$ & 40 & 27.51 & 27.49 & 1.00 \\
\hline $\mathrm{R} 2 / \mathrm{C} / 20$ & 40 & 22.45 & 21.23 & 0.95 \\
\hline & & & Average $P_{p} / P_{t}=$ & 0.96 \\
\hline
\end{tabular}

\begin{tabular}{|c||c|c|c|c|}
\hline $\mathrm{S} / \mathrm{N} / 20$ & 30 & 7.02 & 6.33 & 0.90 \\
\hline $\mathrm{S} / \mathrm{N} / 15$ & 30 & 12.25 & 12.19 & 1.00 \\
\hline $\mathrm{R} 2 / \mathrm{N} / 15$ & 30 & 27.51 & 27.85 & 1.01 \\
\hline $\mathrm{R} 2 / \mathrm{C} / 20$ & 30 & 22.45 & 21.01 & 0.94 \\
\hline
\end{tabular}

\begin{tabular}{|c||c|c|c|c|}
\hline $\mathrm{S} / \mathrm{N} / 20$ & 15 & 7.02 & 6.43 & 0.92 \\
\hline $\mathrm{S} / \mathrm{N} / 15$ & 15 & 12.25 & 12.60 & 1.03 \\
\hline $\mathrm{R} 2 / \mathrm{N} / 15$ & 15 & 27.51 & 28.37 & 1.03 \\
\hline $\mathrm{R} 2 / \mathrm{C} / 20$ & 15 & 22.45 & 19.76 & 0.88 \\
\hline
\end{tabular}

\subsection{Arching within NLFEA models}

Principal stress contour plots from a quarter 3D model of a representative restrained slab (i.e. $R 4 / N / 15)$ are shown for low $\left(P / P_{u l t}=0.37\right)$ and high $\left(P / P_{\text {ult }}=0.84\right)$ levels of loading in Figures 16(a) and 16(b) respectively which illustrate how compressive stresses developed within lower slab regions at supports and in upper slab regions at midspan forming a shallow 'arch' of compression. Principal stress contour plots at selected load levels from a representative restrained slab model are shown in Figures 17(a) and 17(b) which illustrate the development of compressive stresses in lower slab regions close to supports and in upper regions at midspan as loading increased. This behaviour was found to be typical within all 3D solid restrained slab models studied throughout the research. 


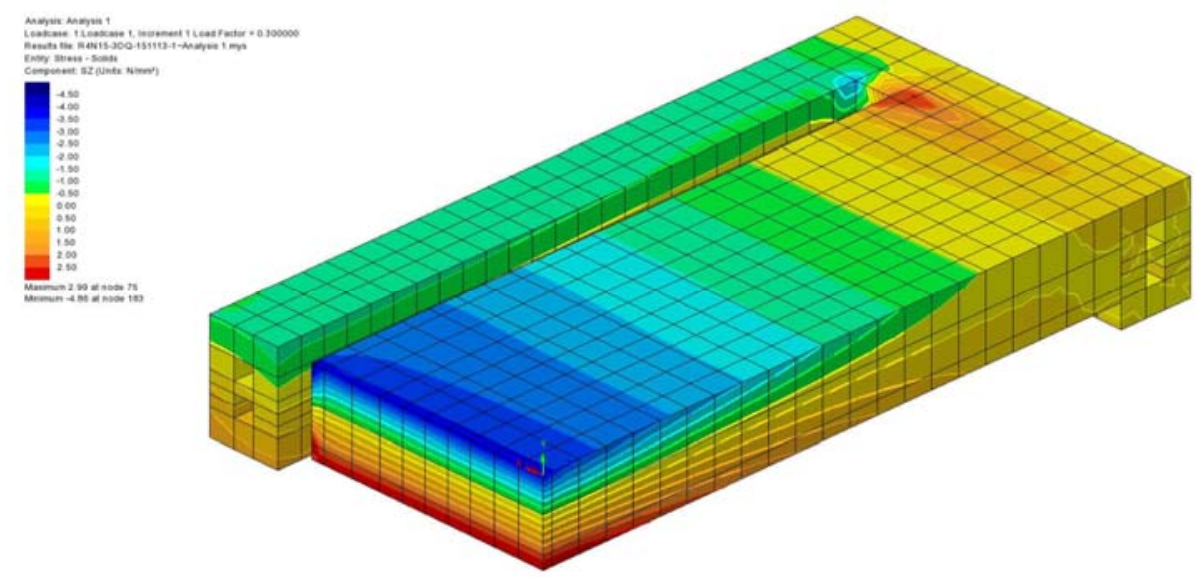

Figure 16(a): Low load level $\left(P / P_{u l t}=0.37\right)$ contour plot of direct $(S Z)$ stresses in concrete within 3D restrained slab model (R4/N/15)

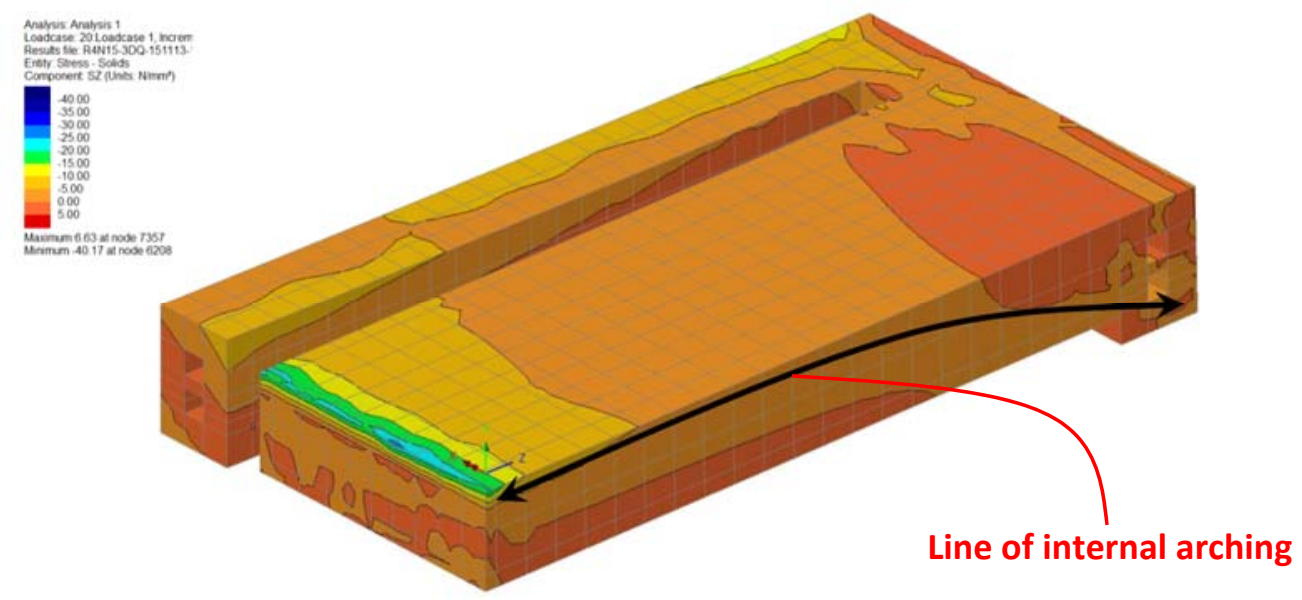

Figure 16(b): High load level (P/Pult=0.84) contour plot of direct (SZ) stresses in concrete within 3D restrained slab model (R4/N/15) 

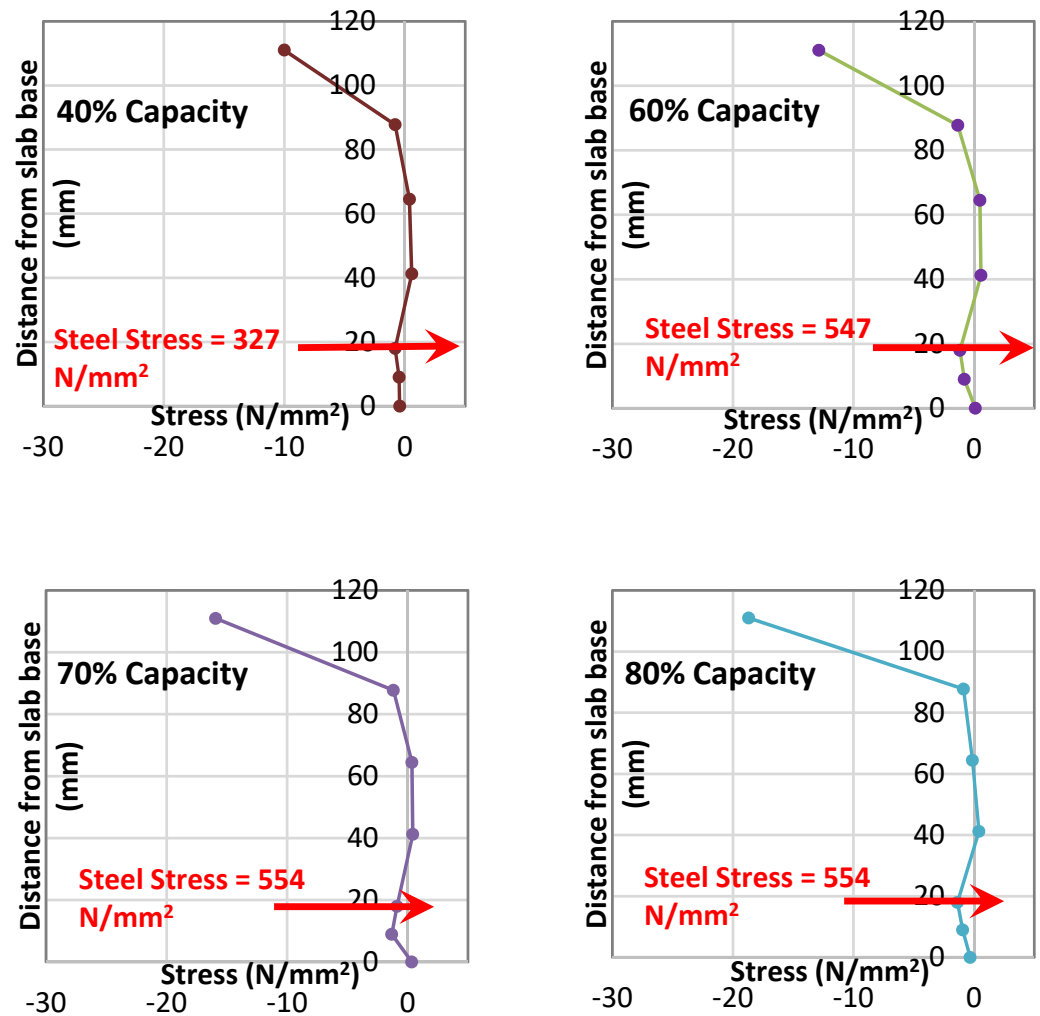

Figure 17(a): Variations in concrete stresses at midspan with depth under increasing proportions of ultimate loading (R4/N/15) [Tension: +ve]
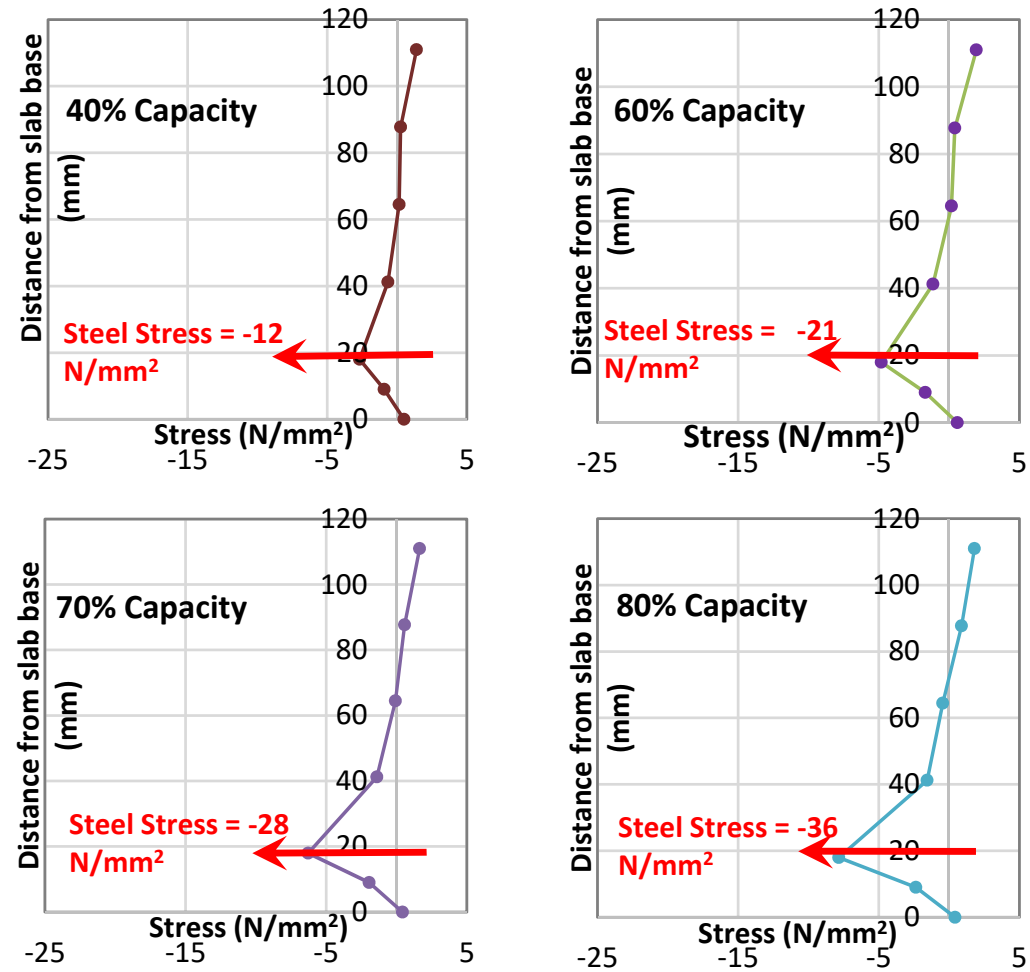

Figure 17(b): Variations in concrete stresses at supports with depth under increasing proportions of ultimate loading (R4/N/15) [Tension +ve] 


\subsection{Capacity prediction and load - deflection performance}

For consistency with experimental investigations, the tensile strength of concrete, $\mathrm{f}_{\mathrm{ct}}$, as established by carrying out tensile cylinder splitting tests [52] was used in primary analyses. However, as the value of $f_{c t}$ was considered to have a significant effect on concrete cracking; and subsequently on ultimate slab capacities, additional NLFEA analyses were also carried out on slab models with upper and lower bound values of $\mathrm{f}_{\mathrm{ct}}$ determined from CEB-FIP Model Code 2010 [63], Neville [64], BS 8007 [65] and Eurocode 2 [28]. These are listed for each slab in Table 10 and show that the experimentally derived cylinder splitting values were approximately halfway between upper and lower limit values from the literature. Analyses therefore allowed investigation into what effect varying $f_{c t}$ had on NLFEA predicted behaviour.

Table 10: Concrete tensile strength values determined from a range of approaches

\begin{tabular}{|c|c|c|c|c|c|c|c|}
\hline Slab Code & $\begin{array}{c}\text { CEB-FIP } \\
\text { [70] } \\
\text { Tensile } \\
\text { Strength } \\
\text { Upper } \\
\text { Limit, } \\
\mathbf{f}_{\text {ctk,max }} \\
\left(\mathrm{N} / \mathrm{mm}^{2}\right)\end{array}$ & $\begin{array}{c}\text { CEB-FIP } \\
\text { [70] } \\
\text { Tensile } \\
\text { Strength } \\
\text { Lower } \\
\text { Limit, } \\
\mathrm{f}_{\mathrm{ctk}, \mathrm{min}} \\
\left(\mathrm{N} / \mathrm{mm}^{2}\right)\end{array}$ & $\begin{array}{c}\text { Neville } \\
\text { [71] \& } \\
\text { BS 8007 } \\
\text { [72] } \\
\text { Tensile } \\
\text { Strength, } \\
\mathbf{f}_{\mathrm{t}} \\
\left(\mathrm{N} / \mathrm{mm}^{2}\right) \\
\end{array}$ & $\begin{array}{c}\text { Eurocode } 2 \\
\text { [28] Mean } \\
\text { Value of } \\
\text { Axial Tensile } \\
\text { Strength, } \mathbf{f}_{\mathrm{ctm}} \\
\left(\mathrm{N} / \mathrm{mm}^{2}\right)\end{array}$ & $\begin{array}{c}\text { Tensile } \\
\text { Splitting Test } \\
\text { Experimentally } \\
\text { Established } \\
\text { Tensile } \\
\text { Strength, } \mathrm{f}_{\mathrm{ct}} \\
\left(\mathrm{N} / \mathrm{mm}^{2}\right)\end{array}$ & $\begin{array}{c}\text { Overall } \\
\text { Upper } \\
\text { Limit on } \\
\text { Concrete } \\
\text { Tensile } \\
\text { Strength } \\
\left(\mathrm{N} / \mathrm{mm}^{2}\right)\end{array}$ & $\begin{array}{c}\text { Overall } \\
\text { Lower } \\
\text { Limit on } \\
\text { Concrete } \\
\text { Tensile } \\
\text { Strength } \\
\left(\mathrm{N} / \mathrm{mm}^{2}\right)\end{array}$ \\
\hline$S / N / 20$ & 4.4 & 2.3 & 1.7 & 3.3 & 3.0 & 4.4 & 1.7 \\
\hline S/N/15 & 4.9 & 2.5 & 1.9 & 3.6 & 3.3 & 4.9 & 1.9 \\
\hline$S / C / 20$ & 4.4 & 2.2 & 1.7 & 3.2 & 3.0 & 4.4 & 1.7 \\
\hline S/B/20 & 5.4 & 2.8 & 2.1 & 4.0 & 3.7 & 5.4 & 2.1 \\
\hline S/B/15 & 4.5 & 2.3 & 1.7 & 3.3 & 3.1 & 4.5 & 1.7 \\
\hline $\mathrm{R} 1 / \mathrm{N} / 20$ & 4.4 & 2.3 & 1.7 & 3.3 & 3.0 & 4.4 & 1.7 \\
\hline $\mathrm{R} 0.5 / \mathrm{N} / 20$ & 4.5 & 2.3 & 1.8 & 3.4 & 3.1 & 4.5 & 1.8 \\
\hline$R 2 / N / 20$ & 4.5 & 2.3 & 1.7 & 3.3 & 3.1 & 4.5 & 1.7 \\
\hline $\mathrm{R} 2 / \mathrm{N} / 15$ & 4.4 & 2.3 & 1.7 & 3.3 & 3.0 & 4.4 & 1.7 \\
\hline R4/N/15 & 4.0 & 2.1 & 1.6 & 3.0 & 2.8 & 4.0 & 1.6 \\
\hline $\mathrm{R} 1 / \mathrm{C} / 20$ & 4.3 & 2.2 & 1.7 & 3.2 & 2.9 & 4.3 & 1.7 \\
\hline R1/B/20 & 4.8 & 2.5 & 1.9 & 3.6 & 3.3 & 4.8 & 1.9 \\
\hline $\mathrm{R} 0.5 / \mathrm{C} / 20$ & 5.0 & 2.6 & 2.0 & 3.7 & 3.4 & 5.0 & 2.0 \\
\hline $\mathrm{R} 0.5 / \mathrm{B} / 20$ & 4.9 & 2.5 & 1.9 & 3.6 & 3.4 & 4.9 & 1.9 \\
\hline $\mathrm{R} 2 / \mathrm{C} / 20$ & 4.3 & 2.2 & 1.7 & 3.2 & 2.9 & 4.3 & 1.7 \\
\hline $\mathrm{R} 2 / \mathrm{B} / 20$ & 4.9 & 2.5 & 1.9 & 3.6 & 3.4 & 4.9 & 1.9 \\
\hline $\mathrm{R} 2 / \mathrm{B} / 15$ & 4.3 & 2.2 & 1.7 & 3.2 & 3.0 & 4.3 & 1.7 \\
\hline
\end{tabular}


Load-deflection output from experiments are presented alongside LUSAS output from models using experimental cylinder splitting $f_{c t}$ values and those carried out with upper and lower bound $f_{c t}$ values from literature are presented and discussed in the following subsections.

\subsubsection{Unstrengthened simply supported (USS) slabs}

Load - deflection outputs for unstrengthened simply supported (USS) slabs are illustrated in Figures 18(a) and 18(b). These show that LUSAS produced reasonable estimates of ultimate capacities, irrespective of the $f_{c t}$ value used, and that initial slab stiffness reduced with decreasing $f_{\text {ct. }}$. However, after initial cracking, NLFEA models of unstrengthened simply supported slabs experienced greater softening than experimental test slabs. NLFEA models employing experimental cylinder splitting $f_{c t}$ values were conservative by approximately $7 \%$ on average.

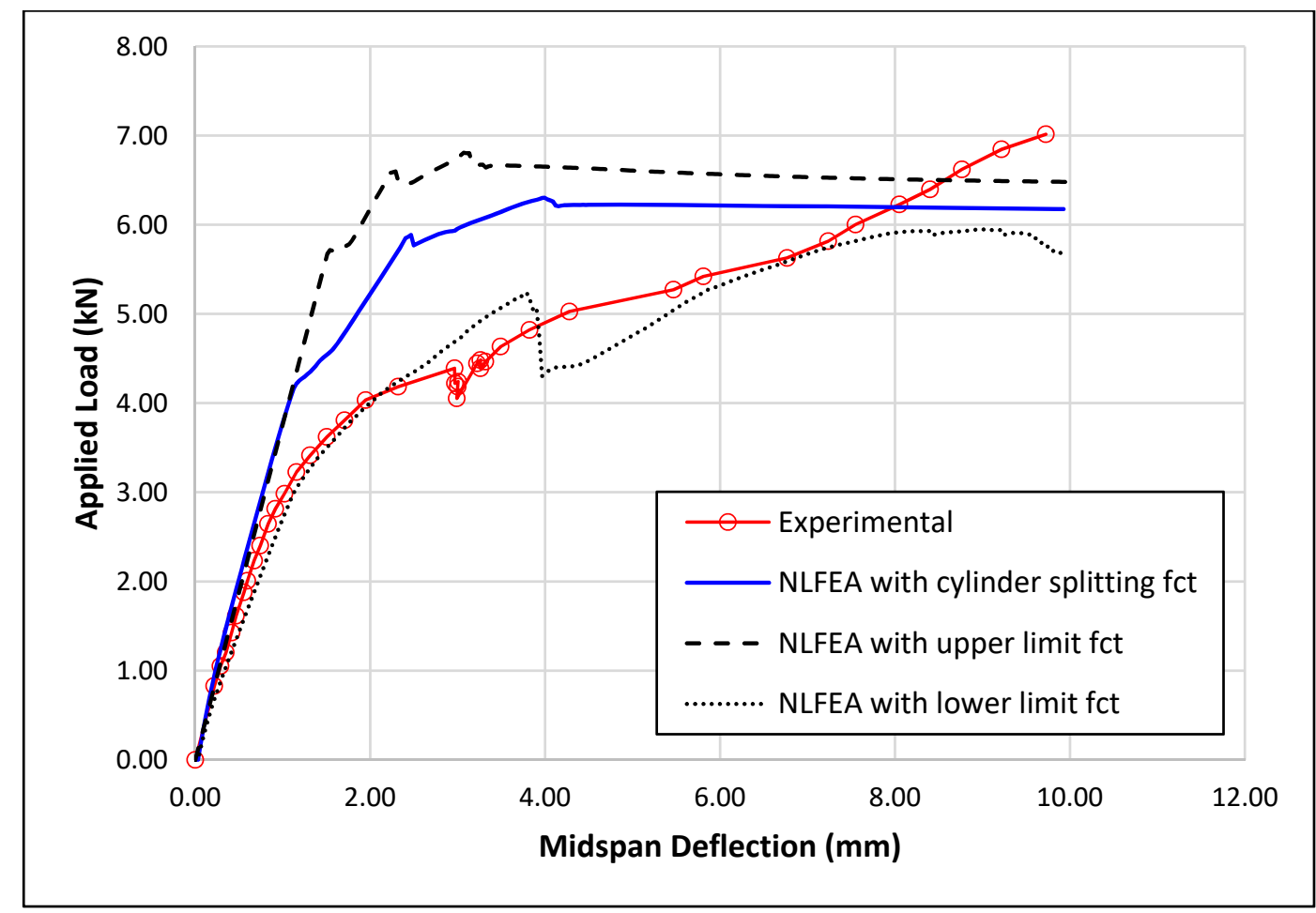

Figure 18(a): Slab S/N/20 load versus deflection results for NLFEA investigations alongside experimental results 


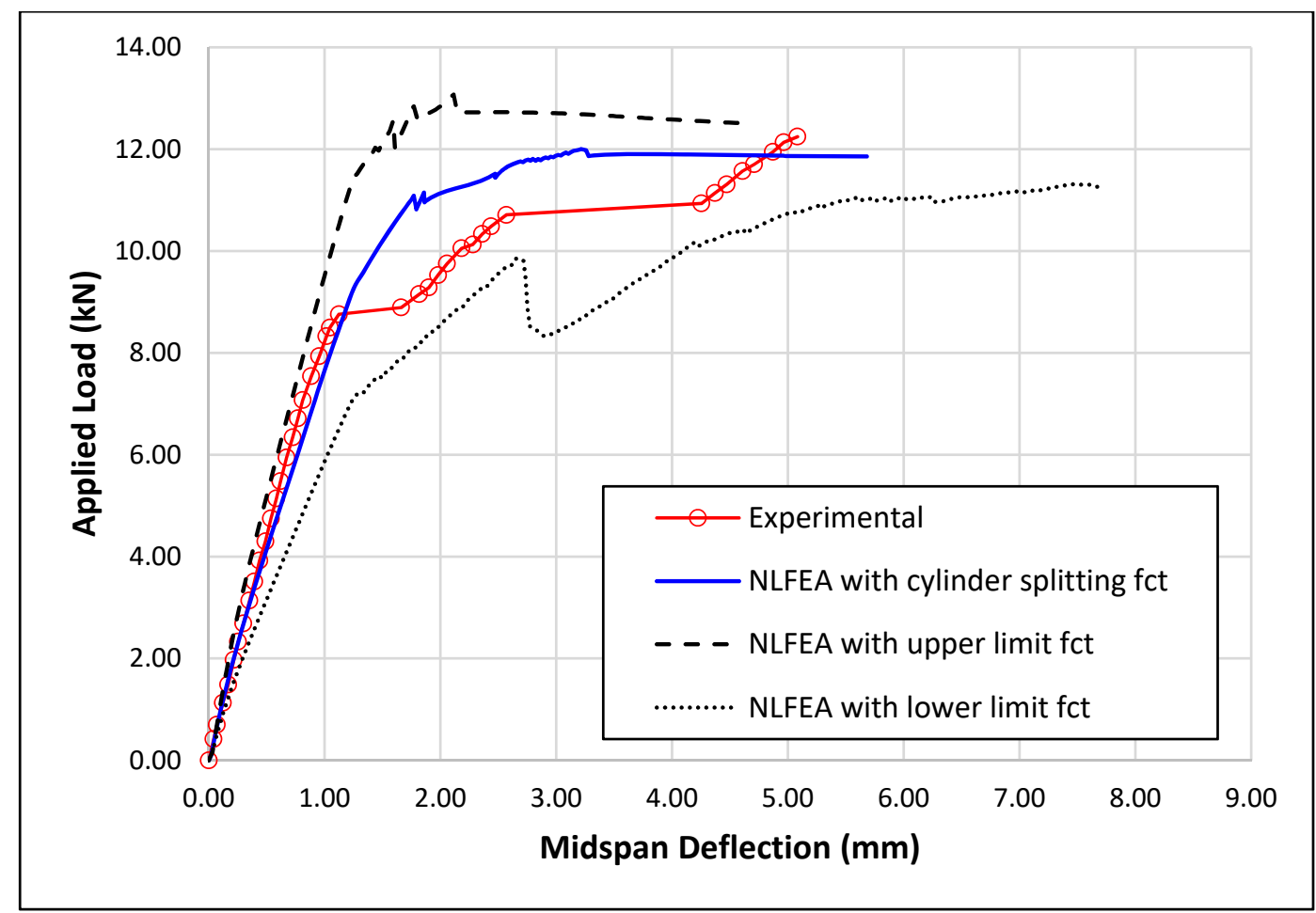

Figure 18(b): Slab S/N/15 load versus deflection results for NLFEA investigations alongside experimental results

\subsubsection{Strengthened simply supported (SSS) slabs}

In strengthened simply supported (SSS) slabs softening due to cracking was more gradual than in USS slabs, resulting in load-deflection trends which were more reflective of experimental behaviour. However, the choice of $f_{c t}$ value appeared to influence the load at which initial cracking occurred and, consequently, the ultimate slab capacity, which is best illustrated by the very close correlation between experimental and LUSAS upper limit $f_{c t}$ results shown in Figure 19(a). Furthermore, where BFRP strengthening was employed, as shown in Figures 19(a) and 19(b), LUSAS predictions using cylinder splitting $f_{c t}$ were lower than experimental results. This could have been due to underestimations of $f_{c t}$ and/or BFRP strength. However, closer agreement between experiments and output from models using upper limit $f_{c t}$ values suggested that $f_{c t}$ had a more significant effect on performance than BFRP strength.

NLFEA predicted capacities for SSS slabs were conservative by approximately $8 \%$ overall. However, in the case of slab $S / C / 20$, shown in Figure 19(c), the predicted capacity exceeded the experimental value by $6 \%$, whilst the overall load-deflection trend for this slab closely followed the experimental behaviour. 


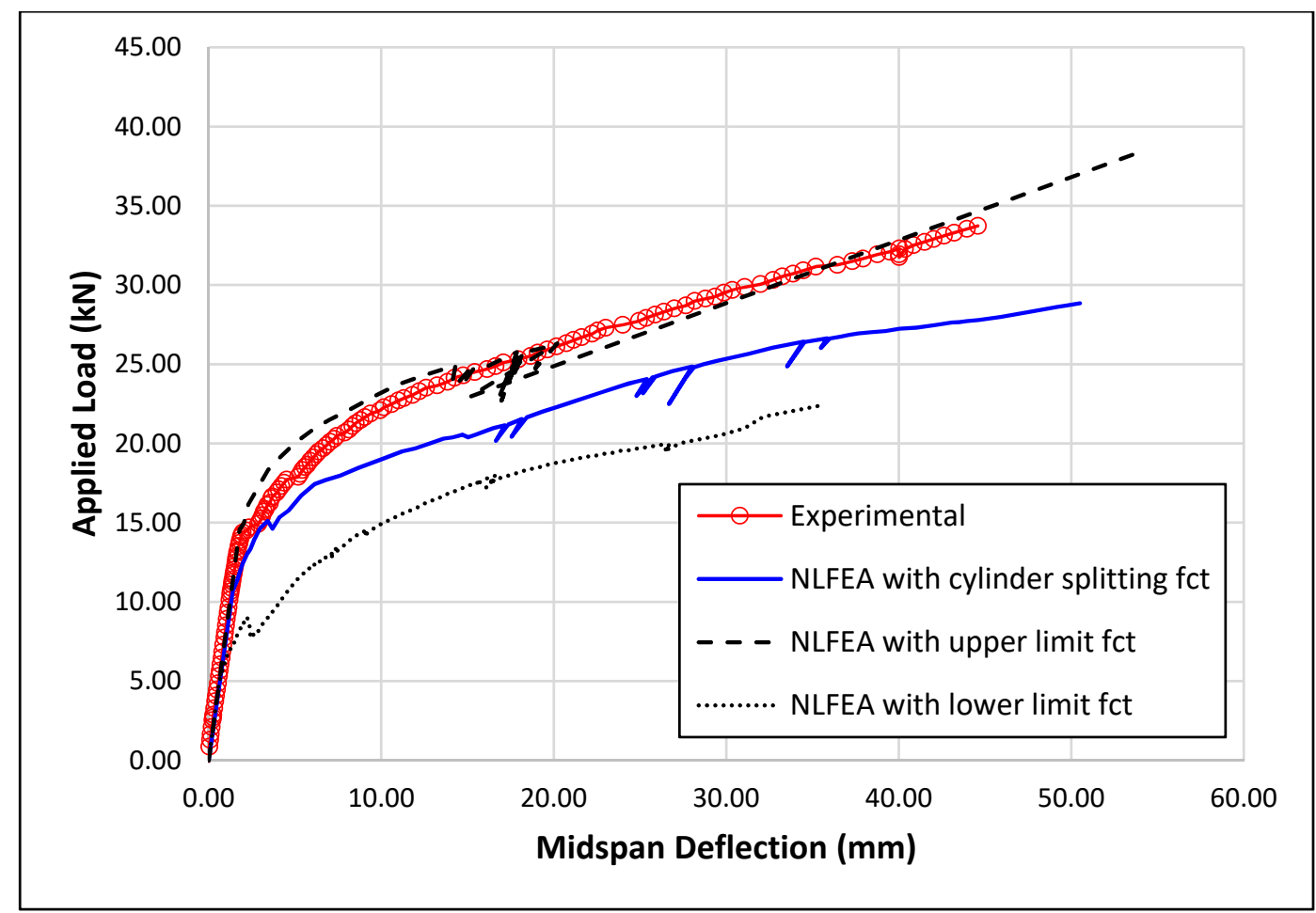

Figure 19(a): Slab S/B/15 load versus deflection results for NLFEA investigations alongside experimental results

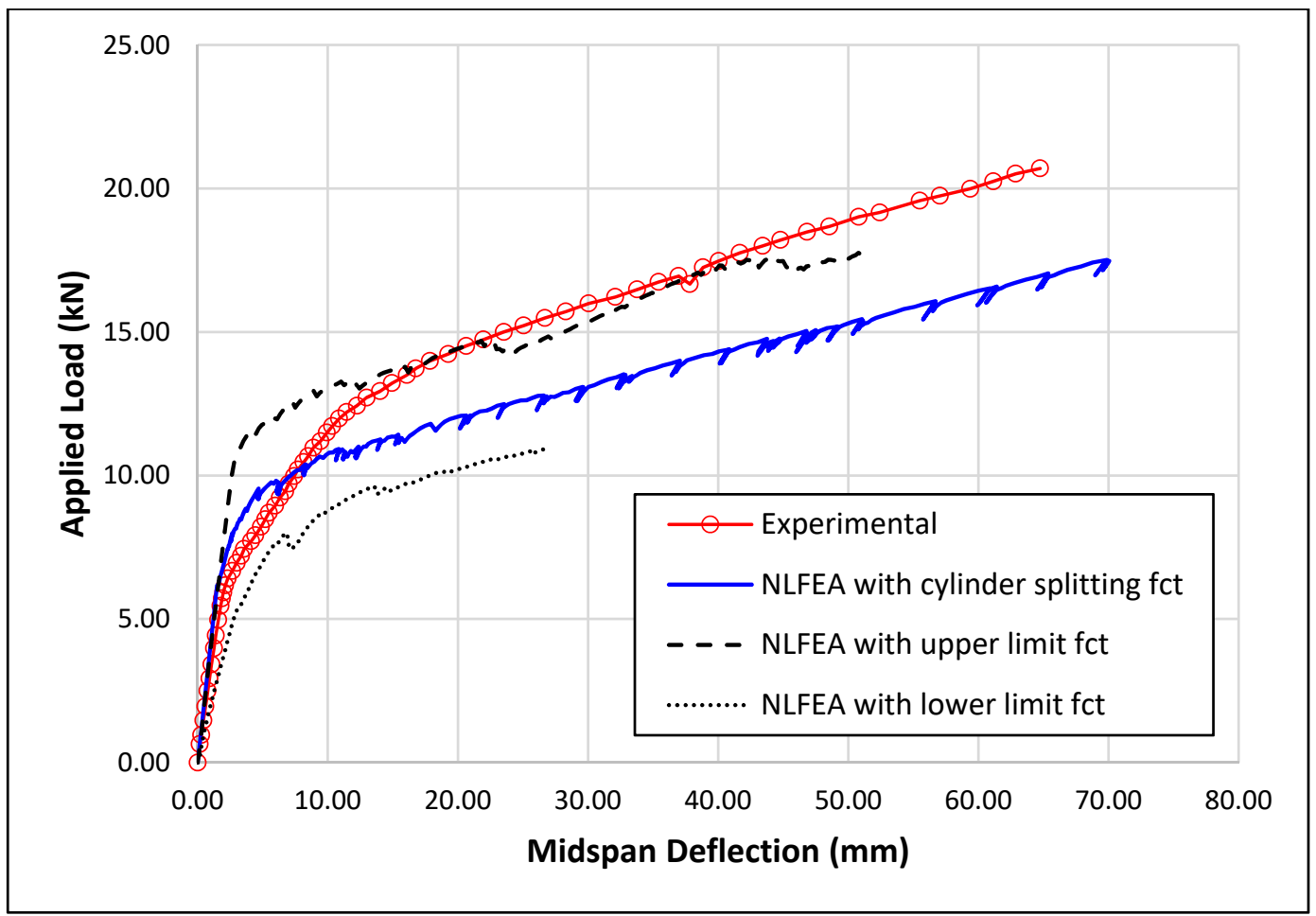

Figure 19(b): Slab S/B/20 load versus deflection results for NLFEA investigations alongside experimental results 


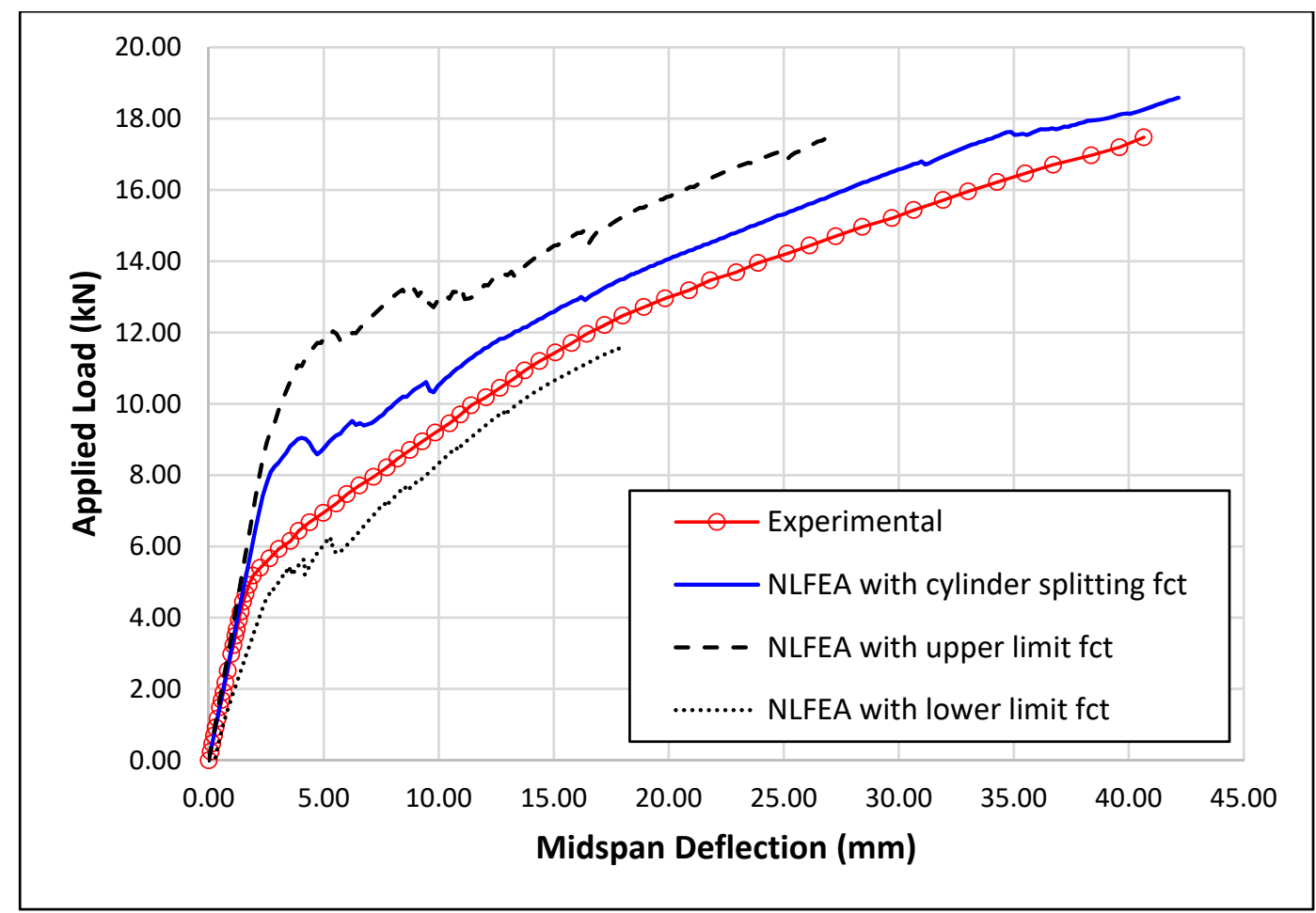

Figure 19(c): Slab S/C/20 load versus deflection results for NLFEA investigations alongside experimental results

\subsubsection{Unstrengthened restrained (UR) slabs}

It can be seen from Figures 20(a), 20(b) and 20(c) that the NLFEA load-deflection behaviour of shallow unstrengthened restrained (UR) slabs was similar to experimental behaviour at low load levels irrespective of the method used in establishing tensile concrete strength, $f_{c t}$. However, with increasing load levels, predicted loads in shallow UR slab models using cylinder splitting $\mathrm{f}_{\mathrm{ct}}$ were consistently higher, at similar deflections, than in experiments for both regular and highly restrained slabs, whilst being near identical in the low restraint sample, as shown in Figure 20(a). In addition, trends from regular and highly restrained shallow UR slabs employing lower bound $f_{c t}$ values were similar to experimental results under low load levels, as shown in Figures 20(b) and 20(c). This suggested that the cylinder splitting $f_{c t}$ values used in these cases were not reflective of concrete in corresponding test slabs and that further refinement of this parameter is recommended. For deep slabs with high and very high restraint, as shown in Figures 20(d) and 20(e), NLFEA predicted loads prior to failure were typically higher, at similar deflections, than corresponding experimental loads. However, these converged to be near identical to experimental values close to ultimate capacity. 
Generally, for UR slabs, NLFEA predicted slab capacities, whilst being very close to experimental values in several cases, were unsafe by approximately $10 \%$ overall on average. It can therefore be concluded that NLFEA modelling can be very effective in predicting the capacities and mid-span deflections of UR slabs, but that establishing the correct value of $f_{c t}$ is of paramount importance in these cases otherwise, inaccurate predictions are likely to be obtained.

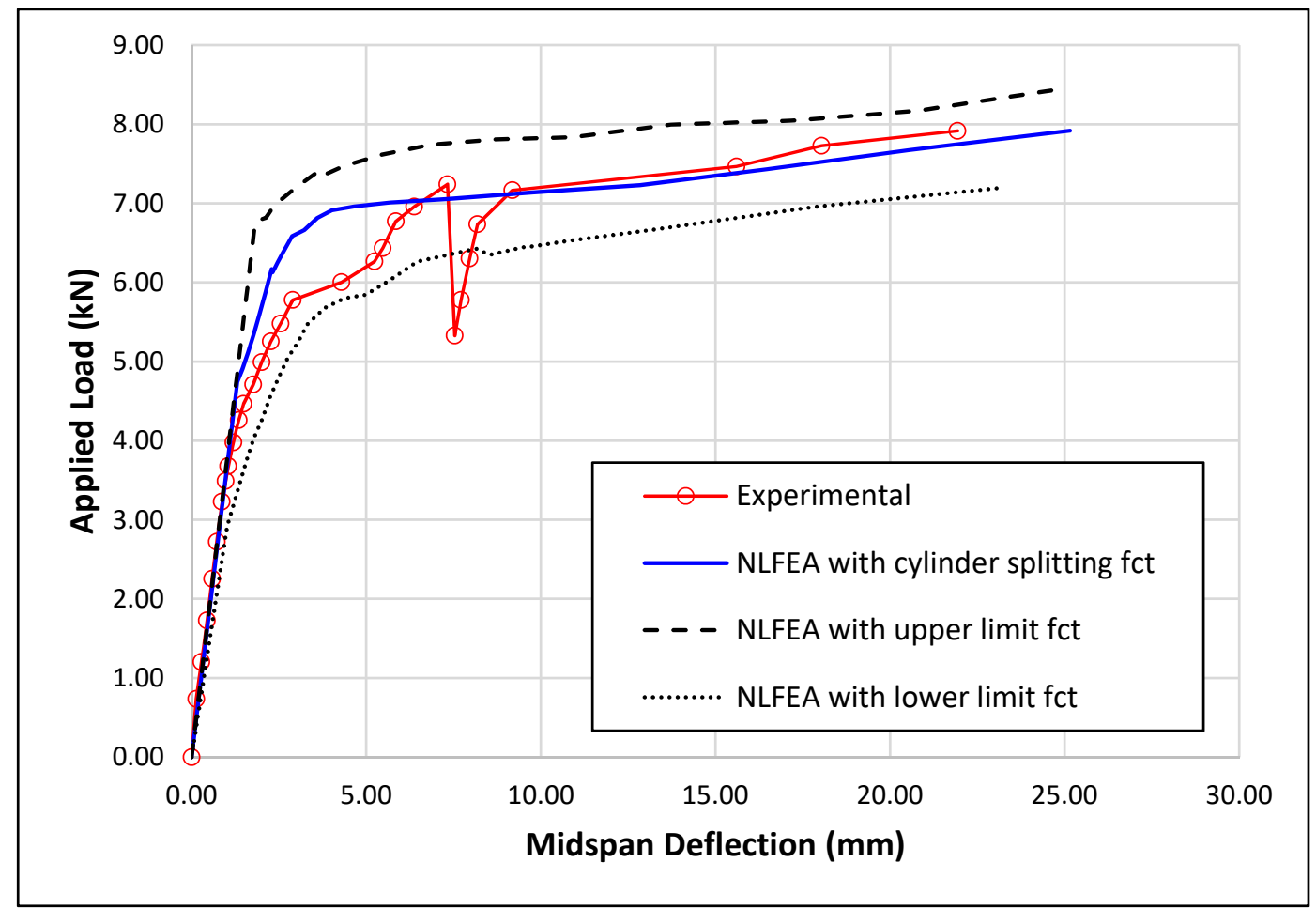

Figure 20(a): Slab R05/N/20 load versus deflection results for NLFEA investigations alongside experimental results 


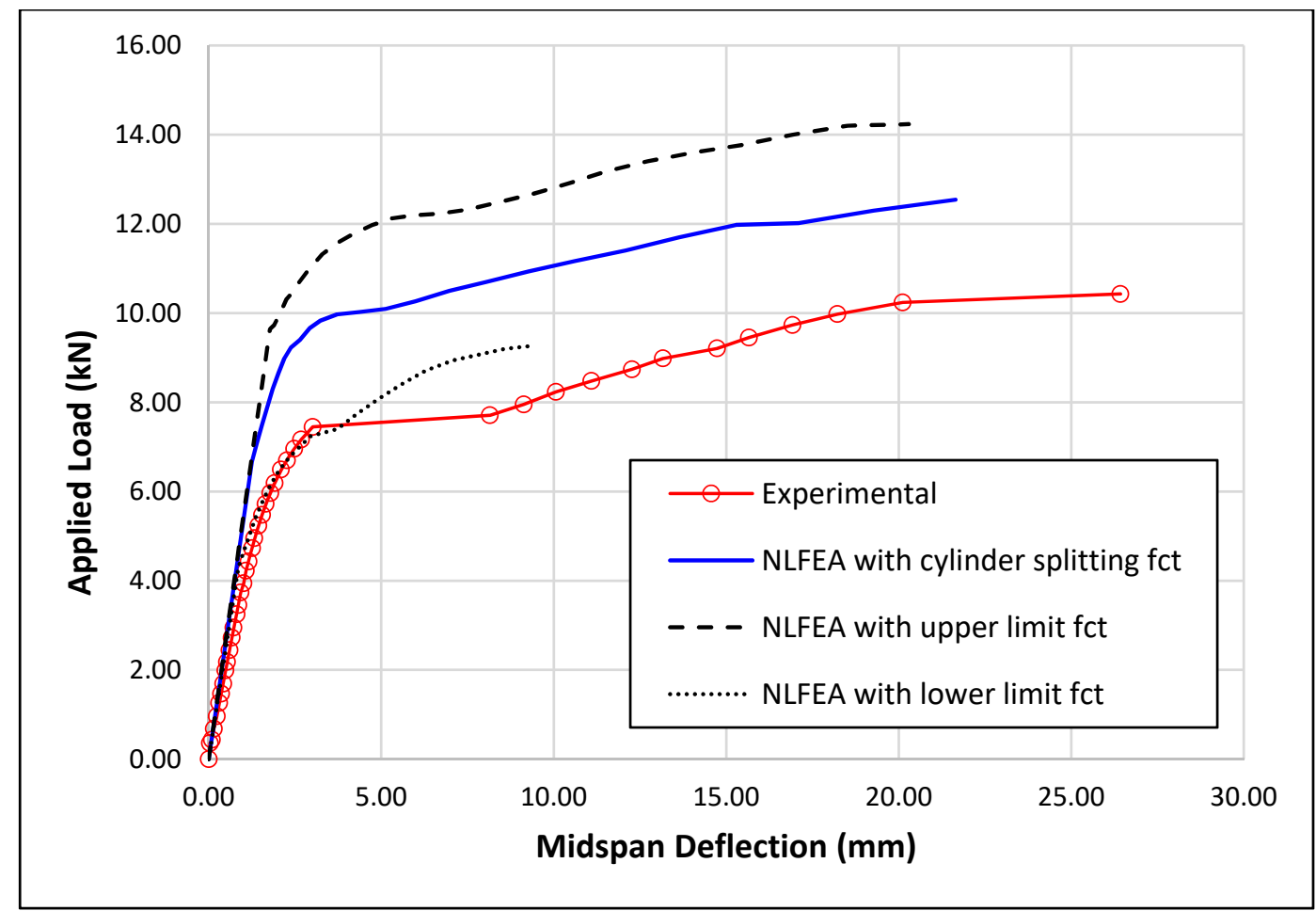

Figure 20(b): Slab R1/N/20 load versus deflection results for NLFEA investigations alongside experimental results

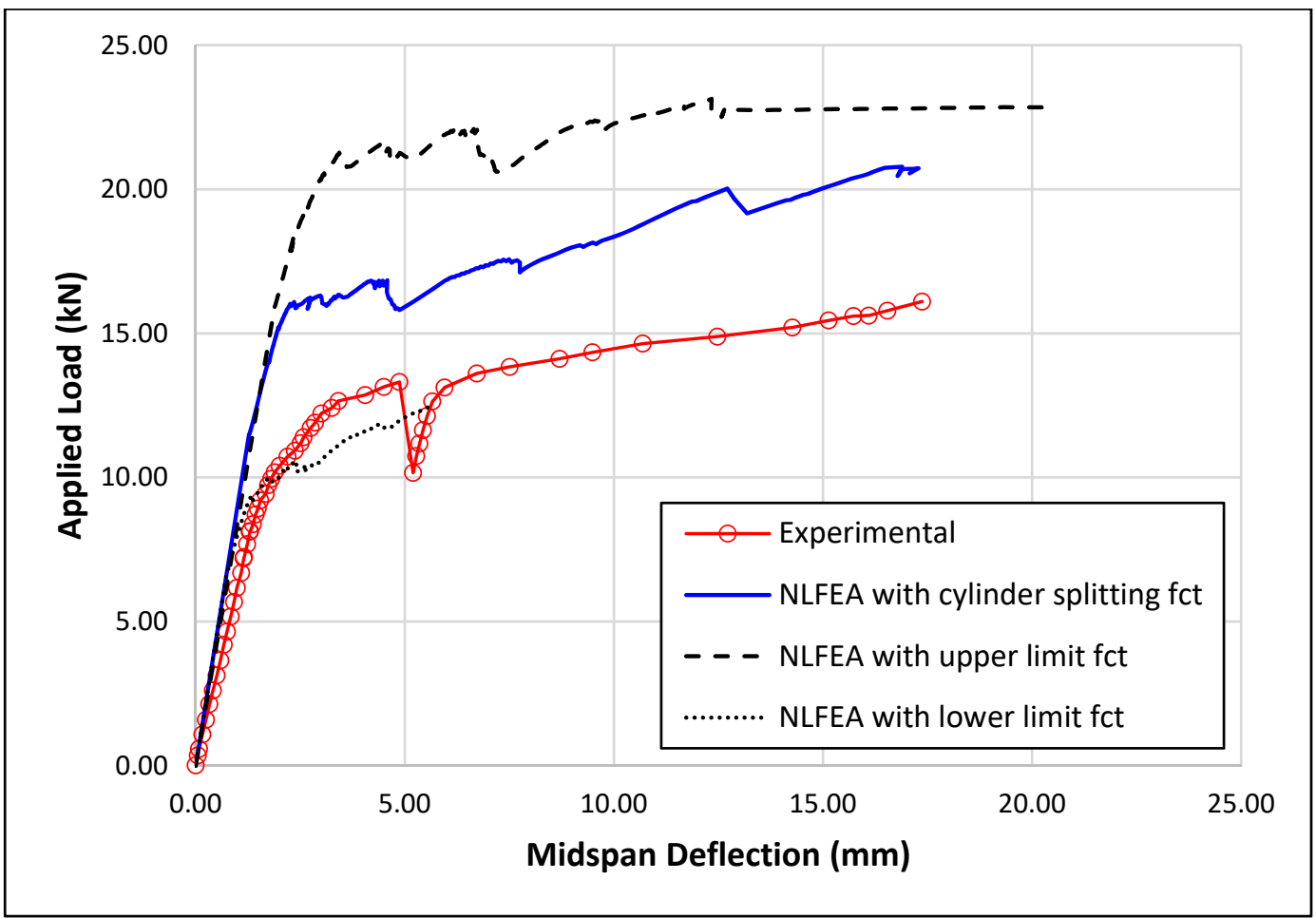

Figure 20(c): Slab R2/N/20 load versus deflection results for NLFEA investigations alongside experimental results 


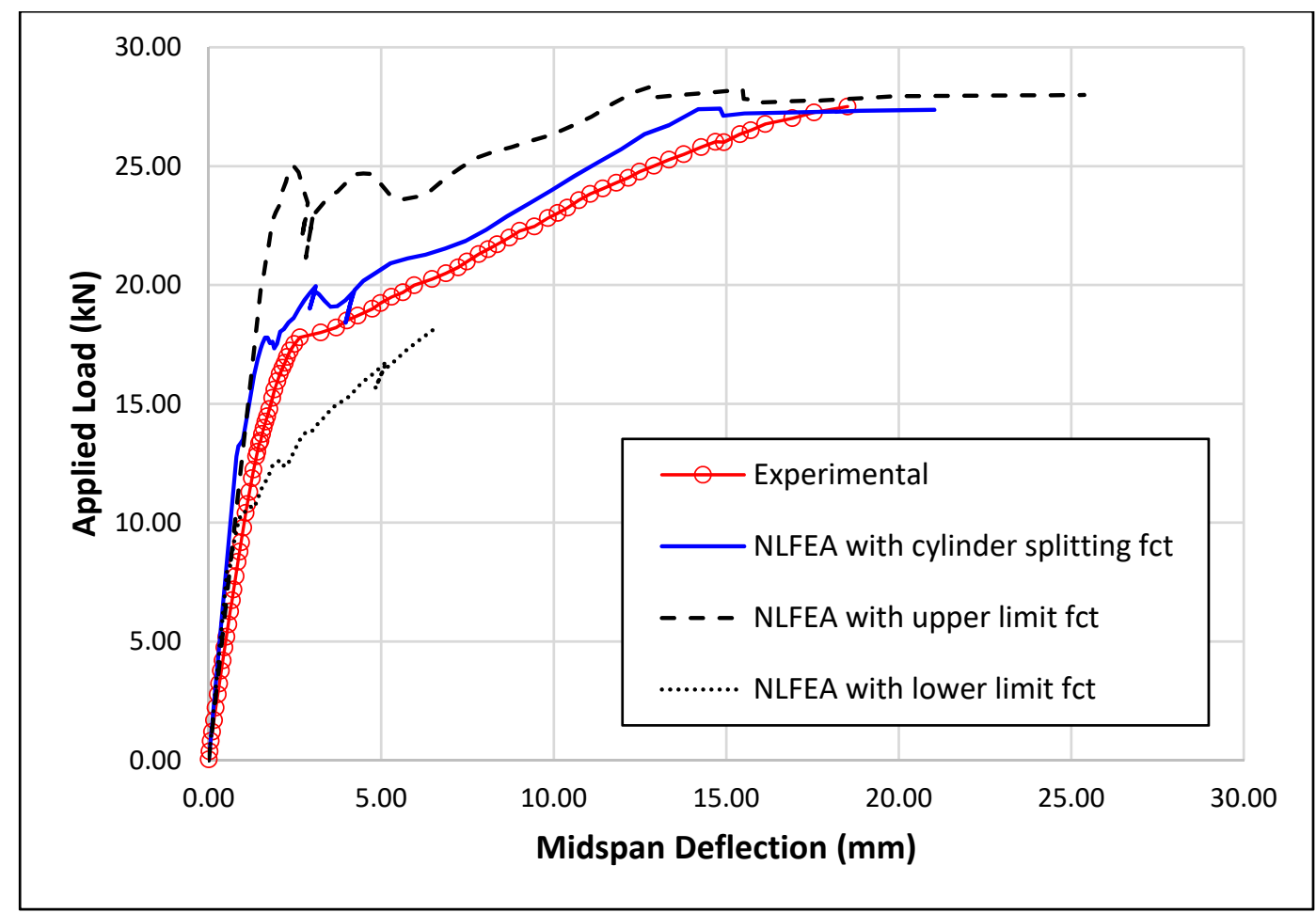

Figure 20(d): Slab R2/N/15 load versus deflection results for NLFEA investigations alongside experimental results

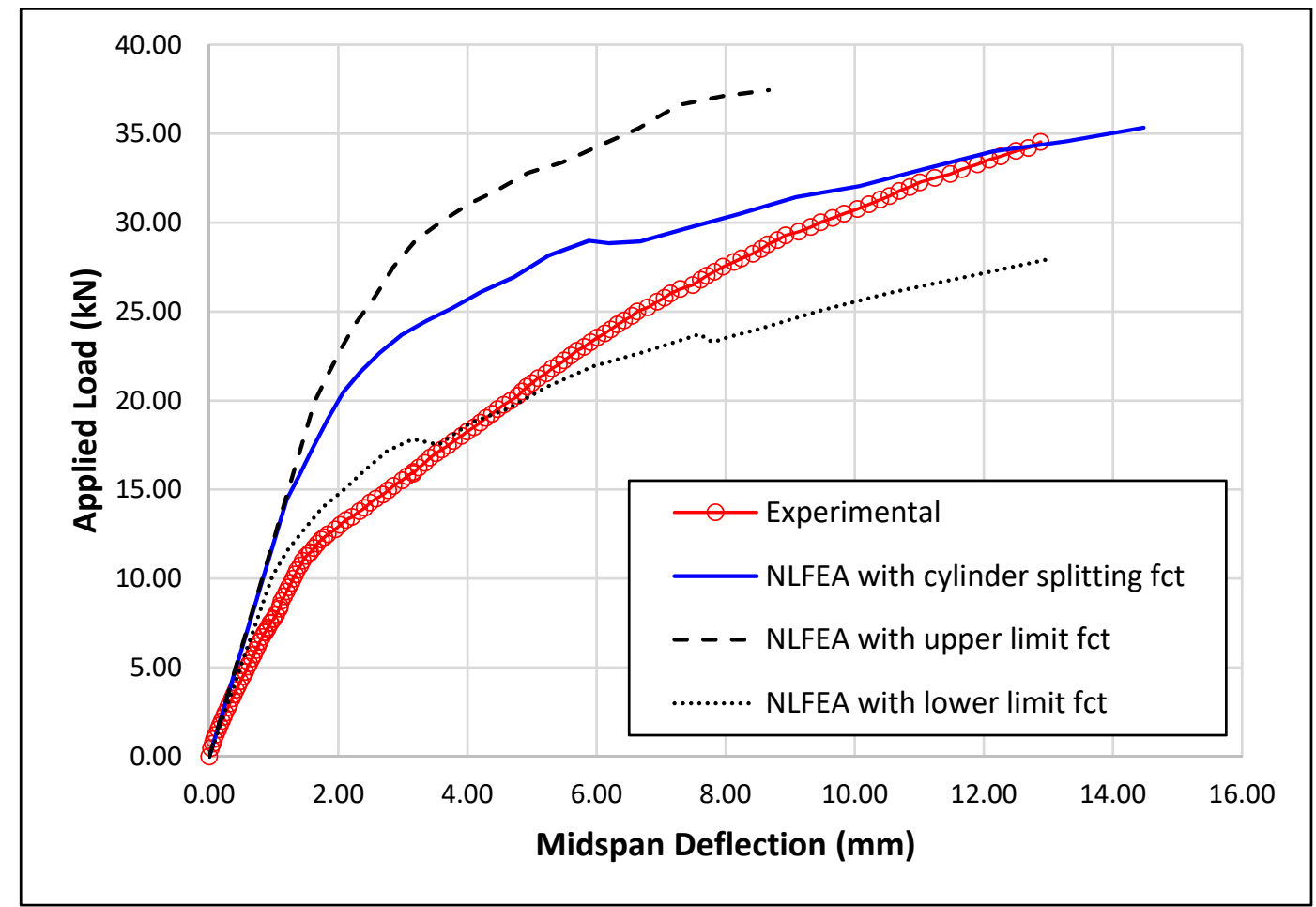

Figure 20(e): Slab R4/N/15 load versus deflection results for NLFEA investigations alongside experimental results 


\subsubsection{Strengthened restrained (SR) slabs}

LUSAS Load - deflection output for strengthened restrained (SR) slabs, using $f_{c t}$ values from cylinder splitting and upper and lower bound limits from the literature, are shown alongside experimental results in Figures 21(a) - 21(g).

In shallow carbon fibre reinforced polymer (CFRP) SR slabs with low and regular levels of in-plane restraint, LUSAS typically overestimated loads compared with experimental results although, where high restraint was used, the load - deflection trend in latter stages of loading corresponded well with experiments. Had all CFRP strengthened models displayed excessive loads, it may have been possible to conclude that the established strength of CFRP bars employed in the research had been overestimated; possibly due to the loading rate during tension testing, mentioned in section 2.1.3, being too high. However, an overestimated value for $f_{c t}$ from cylinder splitting tests was considered to have had a more significant effect, due to more favourable comparisons between experimental trends and output from models employing lower bound $\mathrm{f}_{\mathrm{ct}}$ values.

In basalt fibre reinforced polymer (BFRP) SR slabs, shallow slab models with low restraint and deep slabs with high restraint both displayed load - deflection output which correlated well with experimental behaviour. However, shallow slab models with regular and high restraint produced higher loads, at similar deflections, than experiments. Although, in these cases, output from corresponding lower bound $f_{c t}$ models were similar to experimental results, particularly at low load levels. This may possibly have been due to loading rates during BFRP tension testing, as outlined in section 2.1.3, being too high; leading to an overestimation in BFRP bar strength or that $f_{c t}$ values from cylinder splitting were overestimated.

Overall, estimated SR slab capacities, determined using LUSAS and cylinder splitting $f_{c t}$ values, were unsafe by approximately $7 \%$ on average. However, these overestimations of capacity could not be clearly apportioned to $f_{c t}$ and FRP strength, as variations in both parameters appeared to affect model behaviour. 


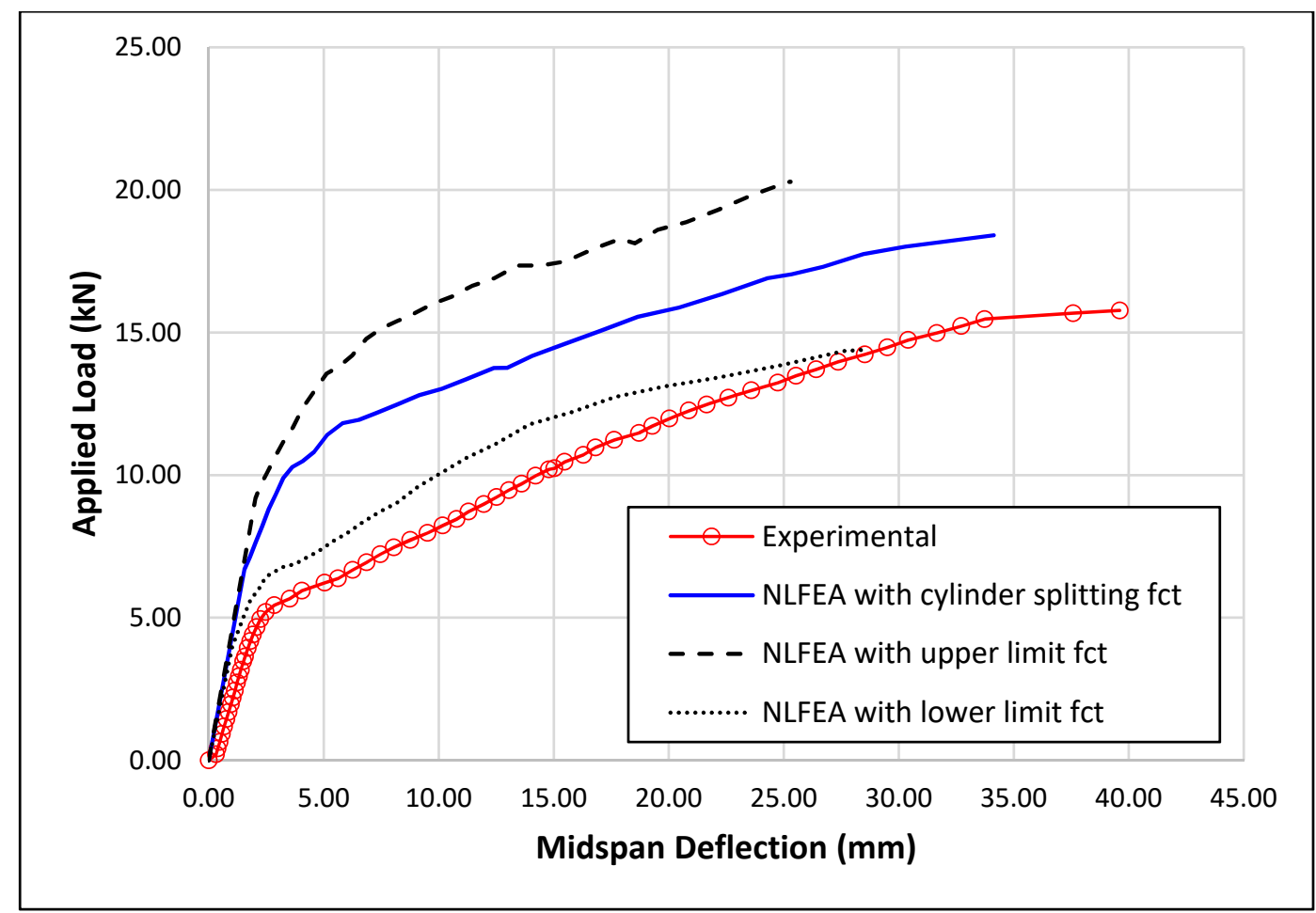

Figure 21(a): Slab R05/C/20 load versus deflection results for NLFEA investigations alongside experimental results

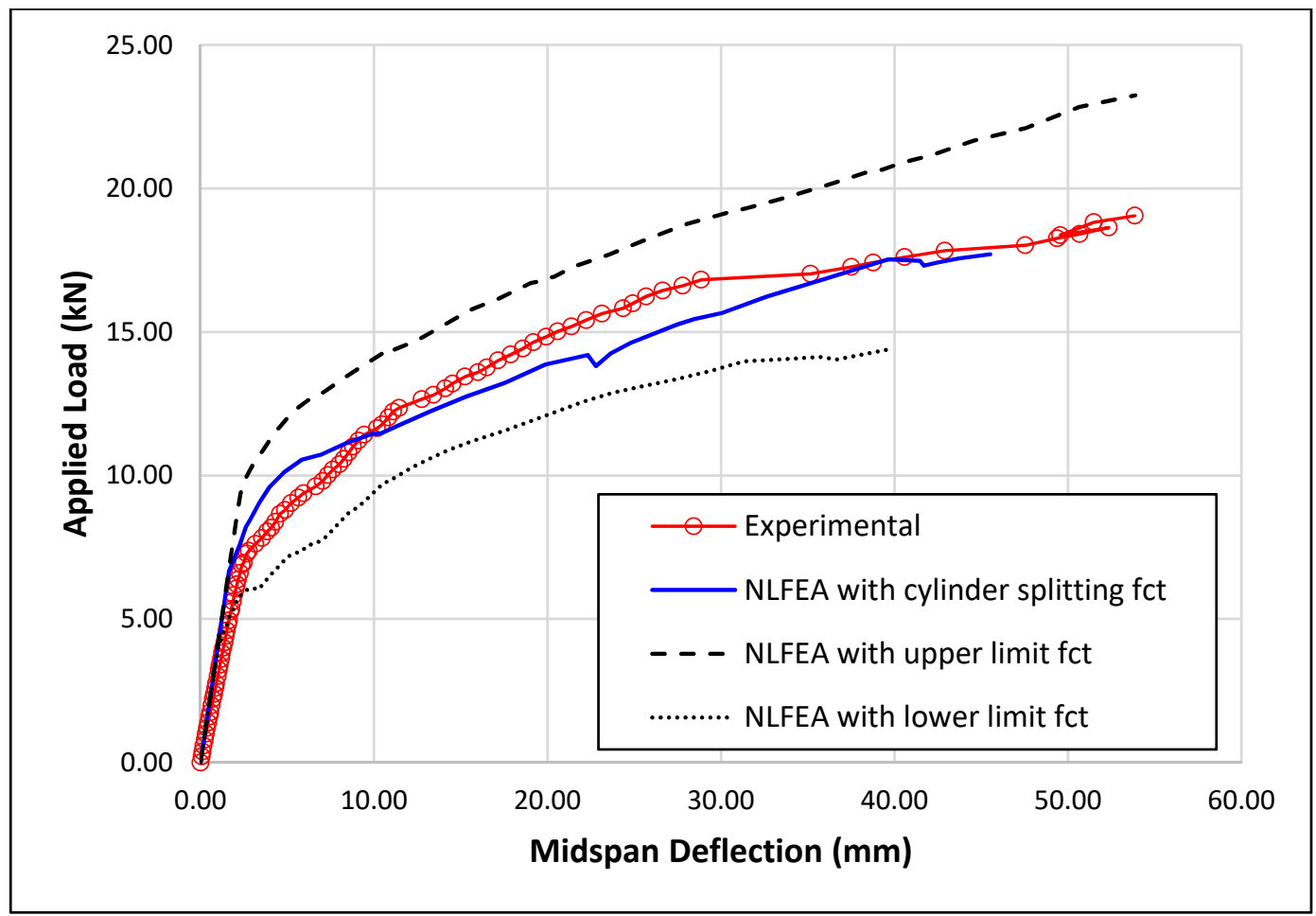

Figure 21(b): Slab R05/B/20 load versus deflection results for NLFEA investigations alongside experimental results 


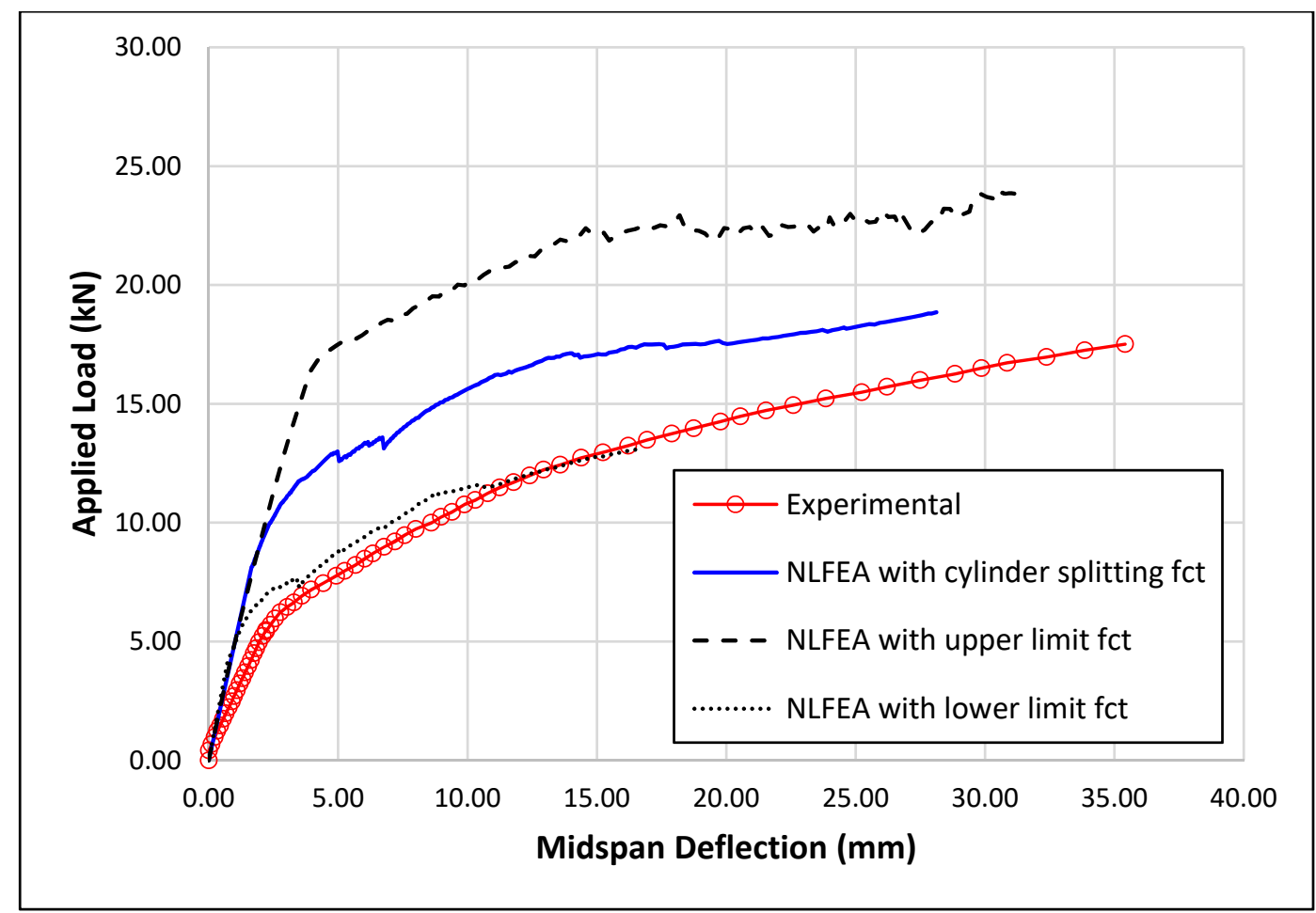

Figure 21(c): Slab R1/C/20 load versus deflection results for NLFEA investigations alongside experimental results

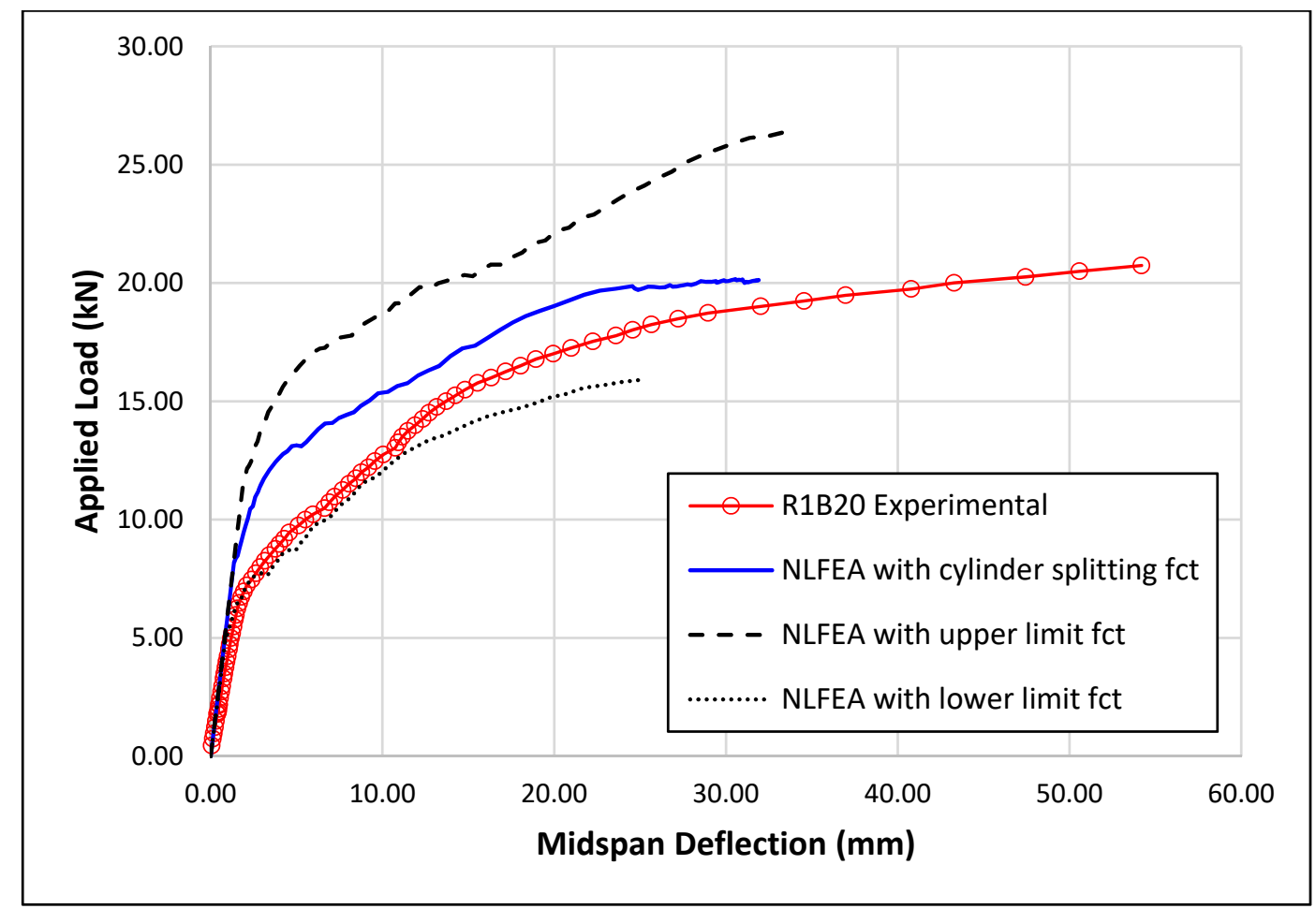

Figure 21(d): Slab R1/B/20 load versus deflection results for NLFEA investigations alongside experimental results 


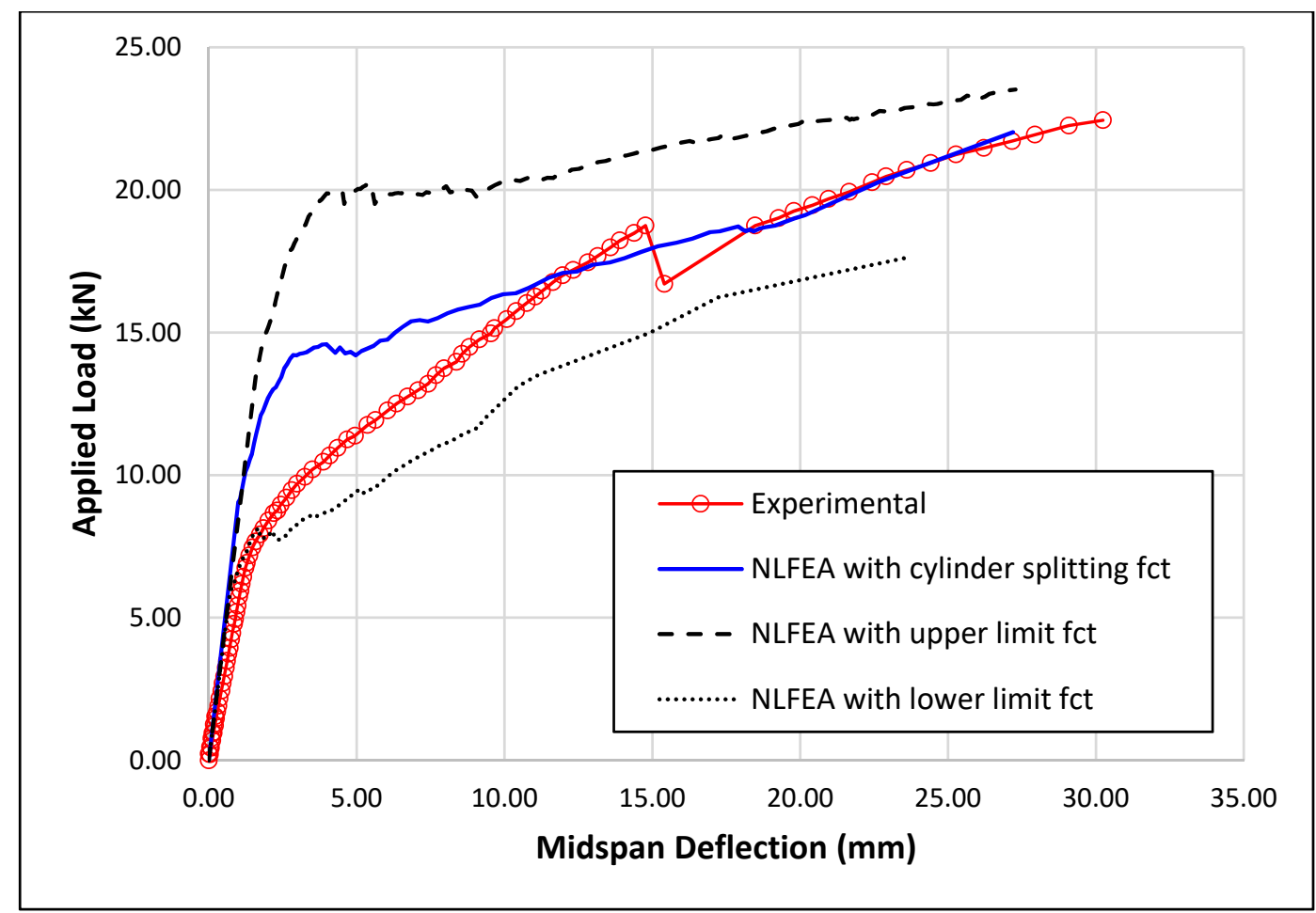

Figure 21(e): Slab R2/C/20 load versus deflection results for NLFEA investigations alongside experimental results

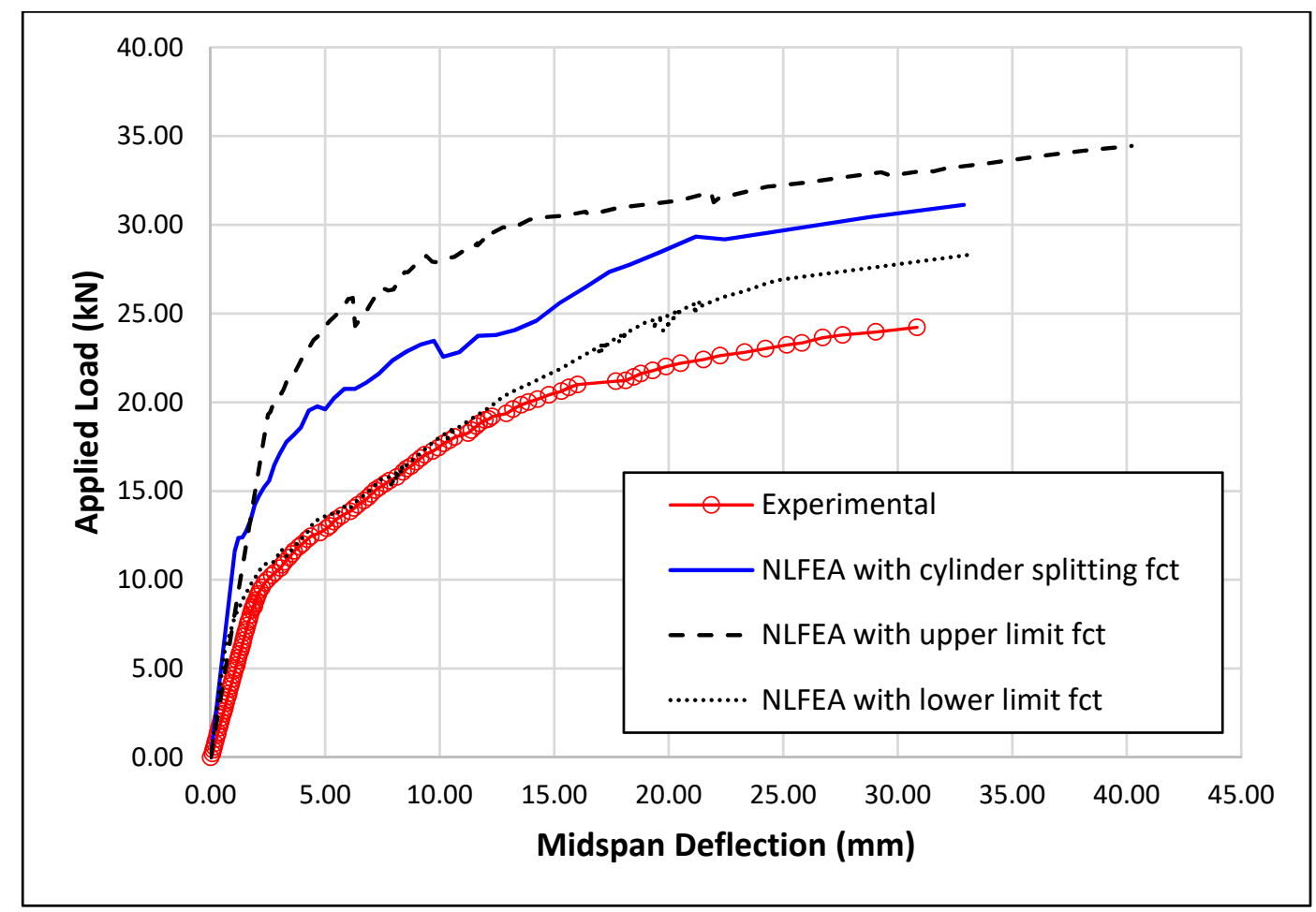

Figure 21(f): Slab R2/B/20 load versus deflection results for NLFEA investigations alongside experimental results 


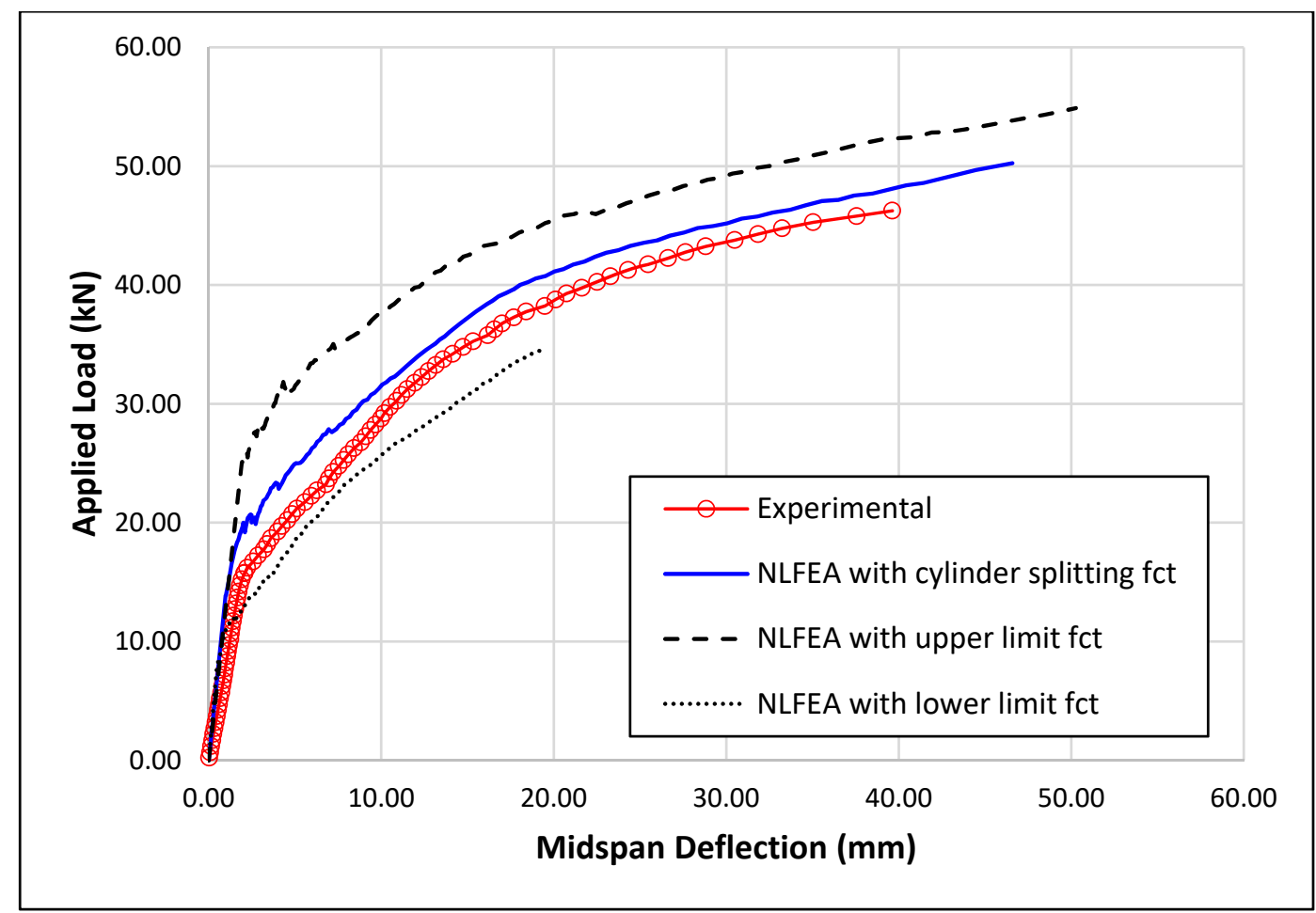

Figure 21(g): Slab R2/B/15 load versus deflection results for NLFEA investigations alongside experimental results

From a comparative inspection of NLFEA output, upper bound $f_{c t}$ estimates were found to be more reflective of experimental behaviour in only two instances (i.e. $S / B / 20$ and $S / B / 15)$, whilst lower bound $f_{c t}$ estimates were more accurate in six cases (i.e. S/N/20, R1/N/20, R2/N/20, R05/C/20, R1/C/20 and R2/B/20). In all remaining instances; i.e. the majority of tests slabs considered in the research; $f_{c t}$ values obtained from experimental cylinder splitting tests contributed towards the most satisfactory NLFEA load-deflection estimates and are therefore recommended for future NLFEA analyses beyond the scope of this work.

Values for the ratio of predicted capacity to test capacity $\left(P_{p} / P_{t}\right)$ are listed in Table 11 from investigations using LUSAS NLFEA methods, Eurocode 2 [28], the American Concrete Institute [29][58] and the Queen's University of Belfast (QUB) Arching Theory [19-26] which allows these approaches to be compared. 
Table 11: Summary of experimental slab capacities alongside predicted capacities using a range of approaches including LUSAS NLFEA

\begin{tabular}{|c|c|c|c|c|c|c|}
\hline Slab Code & $\begin{array}{c}\text { EXPERIMENTAL } \\
\text { FAILURE LOAD (kN) }\end{array}$ & $\begin{array}{l}\text { LUSAS PREDICTED } \\
\text { FAILURE LOAD (kN) }\end{array}$ & $\begin{array}{c}P_{p} / P_{t} \\
\text { (NLFEA) }\end{array}$ & $\begin{array}{l}P_{p} / P_{t} \\
(E C 2)\end{array}$ & $\begin{array}{l}P_{p} / P_{t} \\
(A C l)\end{array}$ & $\begin{array}{c}\mathrm{P}_{\mathrm{p}} / \mathrm{P}_{\mathrm{t}} \\
\text { (Arching) }\end{array}$ \\
\hline$S / N / 20$ & 7.02 & 6.30 & 0.90 & 0.87 & 0.87 & 0.87 \\
\hline$S / N / 15$ & 12.25 & 11.90 & 0.97 & 0.95 & 0.95 & 0.95 \\
\hline$S / C / 20$ & 17.48 & 18.59 & 1.06 & 0.95 & 0.94 & 0.95 \\
\hline S/B/20 & 20.70 & 17.29 & 0.83 & 1.00 & 1.00 & 1.00 \\
\hline S/B/15 & 33.73 & 28.85 & 0.86 & 1.11 & 1.11 & 1.11 \\
\hline $\mathrm{R} 1 / \mathrm{N} / 20$ & 10.43 & 12.55 & 1.20 & 0.58 & 0.58 & 1.26 \\
\hline $\mathrm{R} 0.5 / \mathrm{N} / 20$ & 7.92 & 7.92 & 1.00 & 0.77 & 0.77 & 0.94 \\
\hline $\mathrm{R} 2 / \mathrm{N} / 20$ & 16.11 & 20.71 & 1.29 & 0.38 & 0.38 & 1.06 \\
\hline $\mathrm{R} 2 / \mathrm{N} / 15$ & 27.51 & 27.42 & 1.00 & 0.42 & 0.42 & 0.98 \\
\hline $\mathrm{R} 4 / \mathrm{N} / 15$ & 34.54 & 35.34 & 1.02 & 0.37 & 0.37 & 1.15 \\
\hline $\mathrm{R} 1 / \mathrm{C} / 20$ & 17.52 & 18.85 & 1.08 & 0.95 & 0.94 & 1.16 \\
\hline $\mathrm{R} 1 / \mathrm{B} / 20$ & 20.74 & 20.13 & 0.97 & 1.02 & 1.02 & 1.34 \\
\hline $\mathrm{R} 0.5 / \mathrm{C} / 20$ & 15.77 & 18.41 & 1.17 & 1.06 & 1.05 & 1.12 \\
\hline $\mathrm{R} 0.5 / \mathrm{B} / 20$ & 19.05 & 17.72 & 0.93 & 1.09 & 1.08 & 1.10 \\
\hline $\mathrm{R} 2 / \mathrm{C} / 20$ & 22.45 & 22.03 & 0.98 & 0.74 & 0.73 & 0.97 \\
\hline $\mathrm{R} 2 / \mathrm{B} / 20$ & 24.23 & 31.14 & 1.29 & 0.85 & 0.85 & 1.14 \\
\hline \multirow[t]{4}{*}{$\mathrm{R} 2 / \mathrm{B} / 15$} & 46.26 & 50.27 & 1.09 & 0.84 & 0.81 & 1.00 \\
\hline & \multicolumn{2}{|r|}{ Average $=$} & 1.04 & 0.82 & 0.82 & 1.06 \\
\hline & \multicolumn{2}{|r|}{ Standard Deviation $=$} & 0.14 & 0.25 & 0.24 & 0.13 \\
\hline & \multicolumn{2}{|r|}{ Coefficient of variation $=$} & 0.017 & 0.057 & 0.056 & 0.015 \\
\hline
\end{tabular}

\section{Discussion and conclusions}

The research shows that NLFEA 3D modelling of quarter slabs under deflection control with LUSAS is able to simulate arching behaviour within in-plane restrained slabs and can predict ultimate capacity which is significantly closer to experimental values than those obtained using Eurocode 2 and $\mathrm{ACl}$ methods. This was particularly evident in slabs where FRP strengthening was not employed, as capacity increases due to arching were not overshadowed in these cases by the significant capacity increases attainable through FRP strengthening. Over the range of slabs investigated, LUSAS capacity predictions were slightly (4\%) unsafe on average, but were slightly better than the QUB Arching Theory (6\%). However, it is notable that the hand calculation based QUB arching theory displayed the lowest coefficient of variation of the four methods considered, making it more consistent than the other approaches, 
including the use of NLFEA. However, a further benefit of using LUSAS NLFEA was its ability to provide acceptable midspan load-deflection estimates, which the other methods did not provide.

Reliable results were obtained by LUSAS models by employing HX20 elements for concrete volumes and BRS3 bar elements for both steel reinforcement and FRP strengthening, while NULL elements were applicable to other subdividing lines within each 3D model. Investigations into the sensitivity of the finite element mesh size also showed that optimal results were achieved with a 40mm mesh. Load-deflection output from LUSAS was also satisfactory for all slabs, although it was evident that the chosen $f_{c t}$ value had a significant effect on behaviour. Hence, further investigations into evaluating $f_{c t}$ for the concrete mix and for the slab types used in the research are recommended for application to future NLFEA investigations.

LUSAS modelling therefore represents a valuable and reliable method of simulating slab behaviour irrespective of the presence, or type of FRP strengthening, slab restraint level or slab depth. However, as overestimations in slab capacity occurred due to a combination of concrete and FRP tensile strengths in some models, the use of lower bound strengths and/or stress reduction factors may offer a means of achieving conservative capacity estimates for slabs beyond the range of the variables investigated in this research; although still above those based upon current codified methods.

\section{Acknowledgements}

The authors wish to express their sincere appreciation to MagmaTech for the supply of all basalt and carbon fibre bars used in the research and to The Northern Ireland Department for Employment and Learning (DEL) without which the research could not have been carried out. Further thanks must also go to Professor Paul Lyons and his team from the LUSAS finite element software company and to Professor Tony Jefferson from Cardiff University, for their advice on finite element modelling.

\section{Bibliography}

[1] Kelly, M. (2008) Britain's building stock - a carbon challenge (Presentation, 2008) 
[2] UNEP (2016) UNEP Sustainable buildings and climate initiative: Why buildings. United Nations environment programme (UNEP) (2016) Accessed on January 2016 at http://www.unep.org/sbci/AboutSBCI/Background.asp

[3] Conejos, S., Langston, C., Smith J (2015) Enhancing sustainability through designing for adaptive reuse from the outset: A comparison of adaptSTAR and Adaptive Reuse Potential (ARP) models Facilities, 33 (9/10) (2015), pp. 531-552

[4] Wilkinson, S. J. and Remoy, H., (2017) Adaptive reuse of Sydney offices and sustainability. Sustainable Buildings, 2, 6.

[5] Gopu, V. and Ziehl, P. H., (2016) Strengthening of Bridge Beams using Fiber Reinforced Polymers (FRP). 1st ed. Louisiana, USA: Louisiana Department of Transportation and Development Louisiana Transportation Research Center.

[6] Aidoo J., Harries K.A., Petrou M.F. (2006) Full-Scale Experimental Investigation of Repair of Reinforced Concrete Interstate Bridge Using CFRP Materials. Journal of Bridge Engineering Vol. 11 (No. 3, May 2006) p350

[7] Hawileh, R. A., Rasheed, H. A., Abdalla, J. A., Al-Tamimi, A. K. (2014) Behavior of reinforced concrete beams strengthened with externally bonded hybrid fiber reinforced polymer systems. Materials \& Design, Volume 53, January 2014, Pages 972-982.

[8] Wu, Y., Huang, Y. (2008) Hybrid Bonding of FRP to Reinforced Concrete Structures. Journal of Composites for Construction, Volume 12, Issue 3, pp 266-273.

[9] Sharaky, I. A., Torres, L., Comas, J., Barris, C. (2014) Flexural response of reinforced concrete $(R C)$ beams strengthened with near surface mounted (NSM) fibre reinforced polymer (FRP) bars. Composite Structures, Volume 109, March, Pages 822.

[10] Parvin, A., Syed Shah, T. (2016) Fiber Reinforced Polymer Strengthening of Structures by Near-Surface Mounting Method. Polymers, 8, 298. 
[11] Loring, H. B., Davids, W. G., (2015) Mechanically fastened hybrid composite strips for flexural strengthening of concrete beams. Construction and Building Materials, 76, pp118-129.

[12] Westergaard H. M. and Slater W. A. (1921) Moments and stresses in slabs. J. Am. Conc. Inst. Proc.,1921, 17, pp415-538.

[13] Ockleston A. J. (1955) Load tests on a three-storey reinforced concrete building in Johannesburg. The Structural Engineer, 1955, 33, Oct. 304-322.

[14] Ockleston A. J. (1958) Arching action in reinforced concrete slabs. The Structural Engineer, 1958, 36, No. 6, 197-201.

[15] McDowell, E.L., McKee, K.E., Sevin, E. (1956) Arching Action Theory of Masonry Walls. Proceedings of the American Society of Civil Engineers, Vol. 82, No. ST2, pp915-1 - 918-18.

[16] Park R. (1964a) Ultimate strength of rectangular concrete slabs under shortterm uniform loading with edges restrained against lateral movement. Proceedings of the Institution of Civil Engineers, Volume 28, June, pp125-150.

[17] Park, R, (1964b) Tensile membrane behaviour of uniformly loaded rectangular reinforced concrete slabs with fully restrained edges. Magazine of Concrete Research, Vol. 16, Issue 46, March 1964, pp39-44.

[18] Park, R., (1964c) The ultimate strength and long-term behaviour of uniformly loaded, two-way concrete slabs with partial lateral restraint at all edges. Magazine of Concrete Research, Vol. 16, Issue 48, September 1964, pp139-152.

[19] Rankin G.I.B. (1982) Punching failure and compressive membrane action in reinforced concrete slabs. PhD Thesis, Queen's University Belfast. 
[20] Rankin G.I.B., Long A.E. (1997) Arching action strength enhancement in laterally-restrained slab strips, Proceedings of the Institution of Civil Engineers: Structures and Buildings, Vol. 122, Issue 4 p461-467.

[21] Taylor, S. E. (2000) Compressive Membrane Action in High Strength Concrete Bridge Deck Slabs. PhD Thesis, Queen's University Belfast.

[22] Taylor S.E., Rankin G.I.B., Cleland D.J. (2001) Arching Action in High Strength Concrete Slabs. Proceedings of the Institution of Civil Engineers: Structures and Buildings Vol. 146, Issue 4, p353-362.

[23] Taylor, S.E., Rankin G.I.B. and Cleland D.J. (2002) Guide to compressive membrane action in concrete bridge decks. 1st Ed. Camberley, UK: Concrete Bridge Development Group.

[24] Taylor S.E., Mullin B. (2005) Arching Action in FRP Reinforced Concrete Slabs. Construction and Building Materials (20) p71-80.

[25] Zheng Y., Robinson D., Taylor S., Cleland D., Shaat A. (2008) Analysis of Compressive Membrane Action in Concrete Slabs, Proceedings of the Institution of Civil Engineers: Bridge Engineering, Vol. 161, Issue 1, p 21-31, March 2008.

[26] Tharmarajah, G. (2011) Compressive Membrane Action in Fibre Reinforced Polymer (FRP) Reinforced Concrete Slabs. PhD Thesis, Queen's University Belfast.

[27] BS EN 1990 (2002) Eurocode 2. Eurocode - Basis of structural design, London: BSI Group

[28] BS EN 1992-1-1 (2004) Eurocode 2. Design of concrete structures. General rules and rules for buildings, London: BSI Group

[29] ACl Committee 318-14 (2014) Building Code Requirements for Structural Concrete (ACI 318-14) and Commentary (ACI 318R-14), American Concrete Institute, Farmington Hills, Michigan, USA. 
[30] BD 81/02, (2007) Use of compressive membrane action in bridge decks, Design Manual for Roads and Bridges, Vol. 3, Section 4, Part 20. London: United Kingdom Department for Transport, Highways Agency

[31] American Association of State Highway and Transportation Officials (AASHTO) (2012) Load and Resistance Factor Design (LRFD) Bridge Design Specifications. Washington, D.C, USA

[32] CAN/CSA-S6-06 (2006) Canadian Highway Bridge Design Code, Canadian Standards Association, Toronto, Ontario, Canada, November 2006

[33] Asplund, S. O., (1949) Strengthening Bridge Slabs with Grouted Reinforcement. Journal of the American Concrete Institute, V20 No. 5, Jan pp. 397406

[34] Rasheed H.A., Harrison R.R., Peterman R.J., Alkhrdaji T. (2010) Ductile strengthening using externally bonded and near surface mounted composite systems. Composite Structures (92) p2379-2390.

[35] de Waal, L., Fernando, D., Nguyen, V. T., Cork, R., Foote, J. (2017) FRP strengthening of 60 year old pre-stressed concrete bridge deck units. Engineering Structures, 143, 346-357.

[36] O'Connor, J Alamplali, S Aref A and Trianafilou L (2011) Strategic Development and Deployment of a Composite Bridge Deck, Proceedings of the International conference on Advanced Composites in Construction, UK, 2011.

[37] Militky, J.K., Vladimir, (1996) Ultimate Mechanical Properties of Basalt Filaments. Textile Research Journal, 66(4): p.225-229

[38] American Chemical Society (2003) High Performance Carbon Fibers. [ONLINE] Available at: http://www.acs.org/content/acs/en/education/whatischemistry/landmarks/carbonfiber s.html. [Accessed 17 January 14]. 
[39] Bacon, R. (1960) Growth, Structure and Properties of Graphite Whiskers, Journal of Applied Physics, Vol. 31, No. 2, Feb 1960, p 283-290

[40] Hrennikoff, A. (1942) Solution of problems of elasticity by framework method. Journal of Applied Mechanics, Vol. 9, pp 144-145.

[41] Courant, R. (1943) Variational methods for the solution of problems of equilibrium and vibrations. Bulletin of the American Mathematical Society, Vol. 49 , pp 1-23. CP 114 (1957) B.S. Code of Practice for Reinforced Concrete, London: BSI Group

[42] Turner, M. J., Clough, R.W., Martin, H.C., Topp, L.J. (1956) Stiffness and deflection analysis of complex structures. Journal of the Aeronautical Sciences, Volume 23, Issue 9, pp 805-823 + 854.

[43] Argyris, J. H., (1954) Energy Theorems and Structural Analysis: A Generalized Discourse with Applications on Energy Principles of Structural Analysis Including the Effects of Temperature and Non-Linear Stress-Strain Relations. Aircraft Engineering and Aerospace Technology, Volume 26, Issue 10, 347-356.

[44] Zienkiewicz, O. C., (1947) The Stress Distribution in Gravity Dams. Journal of the Institution of Civil Engineers, Volume 27, Issue 3, pp. 244-271.

[45] Zienkiewicz, O.C. and Gerstner, R. W. (1960) A stress-function approach to interface and mixed boundary-condition problems (boundary conditions and finitedifference techniques). International Journal of Mechanical Sciences, Volume 2, Issues 1-2, 93-101

[46] Zienkiewicz, O.C. and Gerstner, R.W. (1961) The method of interface stress adjustment and its uses in the solution of some plane elasticity problems. International Journal of Mechanical Sciences, Volume 2, Issue 4, 267-276.

[47] Wang, T. M., Lee, S.L. and Zienkiewicz, O.C. (1961) A numerical analysis of large deflections of beams. International Journal of Mechanical Sciences, Volume 3 , Issue 3, 219-228 
[48] Zienkiewicz, O.C. and Cheung, Y.K. (1964). The finite element method for analysis of elastic isotropic and orthotropic slabs. Proceedings of the Institution of Civil Engineers, Volume 28, Issue 4, 471-488.

[49] H.C. Martin (1968) Finite element analysis of fluid flow. Proceedings of the 2nd Conference on Matrix Methods in Structural Mechanics, Wright-Patterson AF Base, Dayton, Ohio.

[50] BS EN 12390-1 (2012) Testing hardened concrete. Shape, dimensions and other requirements for specimens and moulds, London: BSI Group

[51] BS EN 12390-3 (2009) Testing hardened concrete. Compressive strength of test specimens, London: BSI Group

[52] BS EN 12390-6 (2009) Testing hardened concrete. Tensile splitting strength of test specimens, London: BSI Group

[53] BS EN 12390-4 (2000) Testing hardened concrete. Compressive strength. Specification for testing machines, London: BSI Group

[54] Hognestad, E. (1952) Fundamental concepts in ultimate load design of reinforced concrete members, ACI Proceedings, Vol.48, No.10, June 1952, pp.809832.

[55] BS 4449 (2005) Steel for the reinforcement of concrete - Weldable reinforcing steel - Bar, coil and decoiled product - Specification, London: BSI Group

[56] BS EN ISO 15630-1 (2010) Steel for the reinforcement and prestressing of concrete. Test methods. Reinforcing bars, wire rod and wire, London: BSI Group

[57] Tharmarajah, G, Taylor, S.E., Cleland, D. J., Robinson, D. (2014) Corrosionresistant FRP reinforcement for bridge deck slabs. Proceedings of the Institution of Civil Engineers, Bridge Engineering, Volume 11, 1-10. 
[58] ACI 440.2R-17 (2017) Guide for the Design and Construction of Externally Bonded FRP Systems for Strengthening Concrete Structures, American Concrete Institute, Michigan, USA

[59] LUSAS (2015) Modeller Reference Manual. Version 15.0, Issue 2. Surrey, United Kingdom: LUSAS.

[60] LUSAS (2010) Element Reference Manual. Version 14.5, Issue 1. Surrey, United Kingdom: LUSAS.

[61] Concrete Society Working Party (2004) Influence of Tension Stiffening on Deflection of Reinforced Concrete Structures. Concrete Society, UK 2004, Technical Report No. 59.

[62] Bažant ZP, Cedolin L. (1980) Blunt crack band propagation in finite element analysis. Journal of Engineering Mechanics,105(EM2) pp 279-315.

[63] CEB-FIP (2012) CEB-FIP Model Code 2010, Thomas Telford Ltd, London, UK.

[64] Neville, A. M. (1995) Properties of Concrete. 4th Ed. Harlow, England: Pearson Education Limited.

[65] BS 8007 (1987) Design of concrete structures for retaining aqueous liquids, London: BSI Group 